\title{
Topologia algébrica não-abeliana
}

\author{
Renato Vasconcellos Vieira \\ DISSERTAÇÃO APRESENTADA \\ $\mathrm{AO}$ \\ Instituto DE MATEMÁticA E EstatísticA \\ DA \\ Universidade DE SÃo PAUlo \\ PARA \\ OBTENÇÃO DO TÍTULO \\ DE \\ Mestre em CiÊnCIAS \\ Programa: Matemática \\ Orientador: Prof. Dr. Daciberg Lima Gonçalves
}

Durante o desenvolvimento deste trabalho o autor recebeu auxílio financeiro da CAPES e CNPq

São Paulo, Novembro de 2013 



\section{Topologia Algébrica não-abeliana}

Esta versão da dissertação contém as correções e alterações sugeridas pela Comissão Julgadora durante a defesa da versão original do trabalho, realizada em 07/02/2014. Uma cópia da versão original está disponível no Instituto de Matemática e Estatística da Universidade de São Paulo.

Comissão Julgadora:

- Prof. Dr. Daciberg Lima Gonçalves (orientador) - IME-USP

- Profa. Dra. Fernanda Soares Pinto Cardona - IME-USP

- Prof. Dr. Tomas Edson Barros - UfSCar 



\section{Agradecimentos}

A Fernanda Cardona por me acolher e guiar por esse mundo que estou entrando.

Ao Daciberg Gonçalves por também me acolher e sempre me apontar para a direção certa.

Aos professores, funcionários e colegas do IME-USP por criar um ambiente fértil aonde esse trabalho foi possível.

A Erica Martinelli e ao Fernando Rossini por me levantar além de quem eu me imaginava.

Aos meus pais, Elias Vieira e Luiza Vieira, por serem os melhores pais que um filho poderia ter. Vocês me apoiam, me ensinam e proporcionam tantas coisas. Não há palavras o suficiente.

A Fabiana Marchiori que constantemente me lembra que beleza não está no olhar do observador e nem nas coisas. Beleza é o mundo estendendo a mão em sua direção de uma forma que faz parte de você estender a sua de volta.

Ao CNPq e ao CAPES, por ter possibilitado e financiado esta pesquisa. 



\section{Resumo}

VIEIRA, R. V. Topologia algébrica não-abeliana. Dissertação (Mestrado) - Instituto de Matemática e Estatística, Universidade de São Paulo, São Paulo, 2013.

O presente trabalho é uma apresentação de aplicações de estruturas da álgebra de dimensões altas para a teoria de homotopia. Mais precisamente mostramos que existe uma equivalência entre as categorias dos cat ${ }^{n}$-grupos e a dos $n$-cubos cruzados de grupos, ambas equivalentes a categoria das $n$-categorias estritas internas à categoria de grupos, e uma certa subcategoria da categoria dos $n$-cubos fibrantes, os chamados $n$-cubos de Eilenberg-MacLane. Além disso existe uma equivalência entre uma localização dessa subcategoria e a categoria homotópica dos $(n+1)$-tipos homotópicos, o que sugere a utilidade de usar as estruturas algébricas apresentadas como invariantes topológicas. O teorema central dessa teoria, o teorema generalizado de Seifert-van Kampen, diz que o funtor dos $n$-cubos de fibração aos cat $^{n}$-grupos usado para mostrar a equivalência mencionada preserva o colimite de certos diagramas e que nesses casos conectividade é preservada, o que permite certas computações. Apresentaremos definições das estruturas algébricas mencionadas além de como calcular certos colimites na categoria de $n$-cubos cruzados de grupos, demonstraremos os teoremas principais da teoria e mostramos como usar esses resultados para generalizar resultados clássicos da topologia algébrica como o teorema de Blakers-Massey, o teorema de Hurewicz e a fórmula de Hopf para homologia de grupos.

Palavras-chave: $n$-Cubos cruzados de grupos, cat ${ }^{n}$-grupos, teorema generalizado de Seifert-van Kampen, topologia algébrica, teoria de homotopia. 



\begin{abstract}
VIEIRA, R. V. Non-abelian algebraic topology. Dissertation (Master's) - Instituto de Matemática e Estatística, Universidade de São Paulo, São Paulo, 2013.

The present work is a presentation of applications to homotopy theory of structures in higher dimensional algebra. More precisely we show how the categories of crossed $n$-cubes of groups and of cat $^{n}$-groups, both equivalent to the category of strict $n$-categories internal to the category of groups, are equivalent to a subcategory of the category of fibrant $n$-cubes, namely the Eilenberg-MacLane $n$-cubes. There is also an equivalence between a localization of the category of Eilenberg-MacLane $n$-cubes and the homotopy category of homotopy $(n+1)$-types, which suggests the usefulness of the presented algebraic structures as topological invariants. The central theorem of this theory, the generalized Seifert-van Kampen theorem, states that the functor from $n$-cube of fibrations to the cat $^{n}$-groups used to show the aforementioned equivalence preserves the colimit of certain diagrams, and in these cases connectivity is preserved, which permits some computations. We present definitions of the relevant algebraic structures and also how to calculate certain colimits in the category of crossed $n$-cubes of groups, we demonstrate the main theorems of the theory and then we show how to generalize classical results in algebraic topology like the Blakers-Massey theorem, Hurewicz theorem and Hopf's formula for the homology of groups.
\end{abstract}

Keywords: Crossed $n$-cubes of groups, cat $^{n}$-groups, Generalized Seifert-van Kampen theorem, algebraic topology, homotopy theory. 



\section{Sumário}

Sumário $\quad$ viii

$\begin{array}{ll}\text { Lista de Símbolos } & \text { ix }\end{array}$

Lista de Figuras $\quad$ xi

$\begin{array}{ll}\text { Introdução } & \mathbf{1}\end{array}$

Resultados de Whitehead e a sua generalização . . . . . . . . . . . . . . . . . . . 1

Generalização do teorema de Seifert-van Kampen . . . . . . . . . . . . . . . . . . . . 4

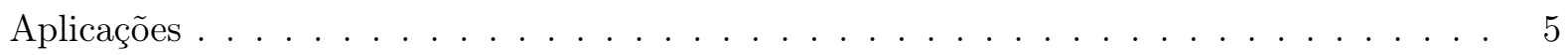

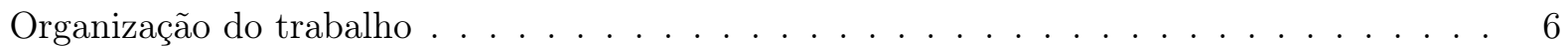

1 Generalizações de módulos cruzados $\quad 9$

$1.1 n$-Cubos cruzados de grupos . . . . . . . . . . . . . . . . 9

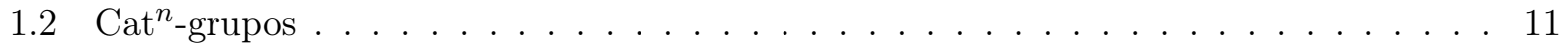

1.3 Equivalência de $n$-cubos cruzados de grupos e cat ${ }^{n}$-grupos $\ldots \ldots \ldots \ldots$

1.4 Resultados categoriais . . . . . . . . . . . . . . . . . . 17

1.5 Módulos cruzados induzidos . . . . . . . . . . . . . . . . . . . 18

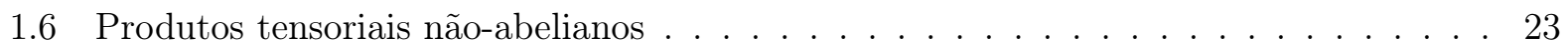

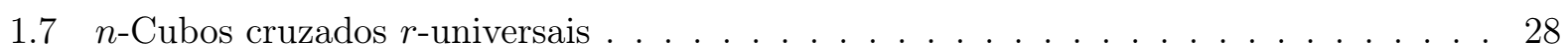

$1.8 n$-Cubos cruzados de grupos $r$-induzidos $\ldots \ldots \ldots \ldots \ldots \ldots \ldots \ldots$

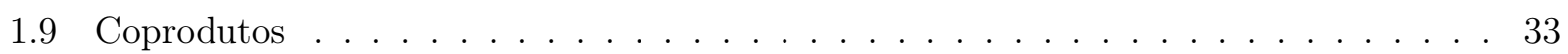

2 n-Cubos de espaços $\quad 35$

$2.1 n$-Cubos fibrantes e de fibrações . . . . . . . . . . . . . . . . . . 35

2.2 Equivalência de $n$-cubos de Eilenberg-MacLane e $(n+1)$-tipos homotópicos $\ldots \ldots 40$

$3 \mathrm{Cat}^{n}$-grupos e $n$-cubos cruzados de grupos fundamentais $\quad 45$

3.1 Homotopia simplicial . . . . . . . . . . . . . . . . . . . . 46

3.2 Cat $^{n}$-grupo cruzado fundamental de um $n$-cubo fibrante . . . . . . . . . . . . 50

3.3 Equivalência de $n$-cubos de Eilenberg-MacLane e cat ${ }^{n}$-grupos . . . . . . . . . . . 53

3.4 Grupos de homotopia do espaço de classificação de um cat ${ }^{n}$-grupo . . . . . . . . . . 57

3.5 Teorema de Seifert-van Kampen generalizado . . . . . . . . . . . . . . . . 60

$3.6 n$-Cubo cruzado de grupos fundamental de um $n$-cubo de fibrações . . . . . . . . . 65

4 Aplicações $\quad 69$

4.1 Aplicações de módulos cruzados induzidos . . . . . . . . . . . . . . . . . . . . . 69 
4.2 Teorema de Blakers-Massey . . . . . . . . . . . . . . . . . . . . 70

4.3 Excisão, teorema de Hurewicz e fórmulas de Hopf . . . . . . . . . . . . . . . . . . . . 72

4.4 Colimites de espaços de classificação de grupos e os grupos de homotopia de $\mathbb{S}^{2} \ldots$

Referências Bibliográficas 


\section{Lista de Símbolos}

$\langle n\rangle \quad$ O conjunto $\{1,2, \ldots, n\}$

$|A| \quad$ A cardinalidade do conjunto $A$

$\underset{d \in D}{\operatorname{colim}}$ O colimite do diagrama $D$

holim O limite homotópico do diagrama $D$

$\subset \quad$ Subconjunto próprio

$\subseteq \quad$ Subconjunto

$\otimes_{\mathbb{Z}} \quad$ Produto tensorial entre grupos abelianos 



\section{Lista de Figuras}

1.13 -cubo cruzado de grupos. . . . . . . . . . . . . . . . . . . . . . 10

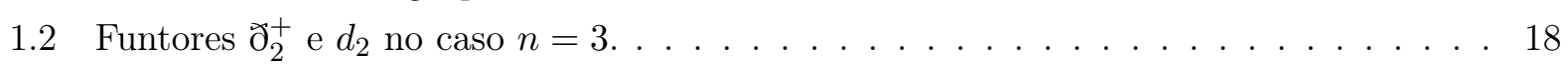

1.3 Diagrama de 3-cubos cruzados de grupos cujo colimite é um 3-cubo cruzado 2-universal. 29

2.13 -cubos de espaços. . . . . . . . . . . . . . . . . . . 36

2.23 -cubo de fibrações. . . . . . . . . . . . . . . . . . 37

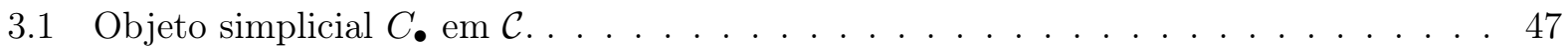

3.2 Objeto 2 -simplicial $C_{\bullet_{1}, \bullet_{2}}$ em $\mathcal{C} \ldots \ldots \ldots \ldots \ldots \ldots \ldots$

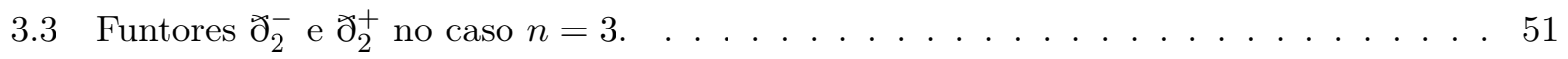

3.43 -cubo cruzado de grupos fundamental de uma quadrupla pontuada. . . . . . . . . . 66

4.1 3-cubo de fibrações associado aos subgrupos normais $N_{1}, N_{2}$ e $N_{3}$ de $G$. . . . . . 74 



\section{Introdução}

Um problema clássico da topologia algébrica é o de classificar as classes de homotopia de CWcomplexos usando modelos algébricos da teoria de grupos. O presente trabalho pretende mostrar uma solução para o problema de classificar as classes de homotopia de CW-complexos que possuem um número finito de grupos de homotopia não triviais utilizando certas estruturas algébricas relacionadas a teoria de categorias de dimensão alta, que tem como partida trabalhos de Whitehead [Whi41, Whi46, Whi49] e tem continuidade no trabalho de Loday [Lod82]. Um dos pontos fortes dessa teoria é que o funtor que relaciona as estruturas topológicas às estruturas algébricas satisfaz uma generalização do teorema de Seifert-van Kampen, o que permite certas computações usando técnicas análogas às usadas para computar grupos fundamentais.

\section{Resultados de Whitehead e a suas generalizações}

É bem conhecido que o funtor $\pi_{1}$, que leva espaços topológicos ao seu grupo fundamental, e o funtor espaço de classificação $B$, que leva um grupo $G$ a um espaço de Eilenberg-MacLane $K(G, 1)$, forma uma equivalência entre a categoria homotópica dos espaços conexos com grupos de homotopia $\pi_{n}$ triviais em dimensões maiores que 1, ou seja os 1-tipos homotópicos conexos, e a categoria dos grupos. O trabalho de Whitehead [Whi41, Whi46, Whi49] de certa forma estende esse resultado e nos dá a estrutura algébrica que modela os 2-tipos homotópicos. Para obter essas estruturas é necessário escolher uma fibração apropriada. Vejamos brevemente as ideia básicas contidas em seu trabalho, e como elas foram generalizadas.

Seja $X$ um espaço com ponto base $x_{0}$ e $U$ um subespaço com o mesmo ponto base. Temos então uma sequência exata desse par

$$
\cdots \rightarrow \pi_{q+1}(X ; U) \rightarrow \pi_{q}(U) \rightarrow \pi_{q}(X) \rightarrow \cdots
$$

que é a sequencia exata da fibração $f: E_{U} \rightarrow X$ associada a inclusão do subespaço $U$ em $X$, aonde $E_{U}$ é o espaço dos caminhos $\gamma \in X^{I}$ em $X$ com $\gamma(1) \in U$, e aonde $f(\gamma)=\gamma(0)$ [May99, Cap. 7]. Note que $E_{U}$ retrai por deformação em um espaço homeomorfo a $U$, e que a fibra $F_{U}$ é dada por caminhos em $X$ que começam no ponto base $x_{0}$ e terminam em algum ponto de $U$. Temos então que os elementos de $\pi_{2}(X ; U)=\pi_{1}\left(F_{U}\right)$ são dados por aplicações $\psi: I \times I \rightarrow X$ tal que os pontos da forma $(0, t),(1, t)$ e $(t, 0)$ são levados ao ponto base e os pontos da forma $(t, 1)$ são levados em $U$. A operação de grupos é dada por concatenação na "direção horizontal", ou mais precisamente pela primeira coordenada.

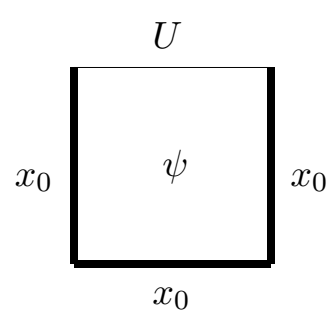


O homomorfismo induzido pela aplicação de bordo $\partial: \pi_{2}(X ; U) \rightarrow \pi_{1}(U)$ é dada num elemento $\psi$ como acima pela restrição em $I \times\{1\}$.

Temos uma ação de $\pi_{1}(U)$ nos elementos de $\pi_{2}(X ; U)$ que pode ser ilustrada da seguinte forma. Dado um elemento $\alpha \in \pi_{1}(U)$ e um elemento $\psi \in \pi_{2}(X ; U)$ como acima o elemento ${ }^{\alpha} \psi$ pode ser visto como uma redução do domínio de $\psi$ a um quadrado menor e então colocando o caminho $\alpha$ nos segmentos radiais fora desse quadrado como no desenho abaixo.

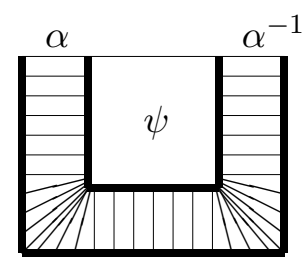

Além disso sempre valem as equações

$$
\begin{gathered}
\partial\left({ }^{\alpha} \psi\right)=\alpha \partial(\psi) \alpha^{-1} \\
\phi \psi \phi^{-1}={ }^{\partial(\phi)} \psi
\end{gathered}
$$

Whitehead denotou como módulo cruzado qualquer homomorfismo de grupos $\mu: M \rightarrow G$ aonde $G$ age em $M$ e as equações acima são satisfeitas. Um morfismo entre módulos cruzados é um diagrama comutativo

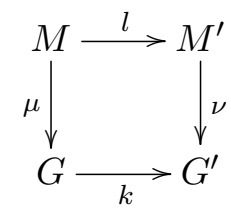

aonde os homomorfismos verticais são módulos cruzados e o par $(k, l)$ preserva a ação, no sentido que $l\left({ }^{g} m\right)={ }^{k(g)} l(m)$. O módulo cruzado formado pelo homomorfismo $\partial: \pi_{2}(X ; U) \rightarrow \pi_{1}(U)$ e pela ação de $\pi_{1}(U)$ em $\pi_{2}(X ; U)$ descrita acima é denotado como o módulo cruzado fundamental do par $(X ; U)$. Essa construção nos dá um funtor

\section{$\Pi$ : pares pontuados de espaços $\rightarrow$ módulos cruzados}

sendo que pelo trabalho de Quillen [Qui67] podemos substituir a categoria dos pares de espaços pontuados pela a categoria mais geral das fibrações.

Para ver a relevância do conceito de módulo cruzado para a teoria de homotopia apontamos que pelo resultado de Mac Lane e Whitehead [MW50] existe um funtor $B$, também chamado de funtor espaço de classificação, que associa a um módulo cruzado $\mathcal{M}=(\mu: M \rightarrow G)$ um CW-complexo pontuado conexo denotado $B(\mathcal{M})$ com as seguintes propriedades:

Teorema. Os grupos de homotopia de B(M) são dados por

$$
\pi_{i}(B(\mu: M \rightarrow G)) \cong \begin{cases}\text { Coker } \mu & \text { para } i=1 \\ \operatorname{Ker} \mu & \text { para } i=2 \\ 0 & \text { para } i>2\end{cases}
$$

Teorema. O espaço de classificação $B(1 \rightarrow G)$ é o espaço de classificação $B(G)$ usual do grupo $G$, 
e $B(G)$ é um subcomplexo de $B(\mu: M \rightarrow G)$. Além disso existe um isomorfismo natural de módulos cruzados

$$
\Pi(B(\mu: M \rightarrow G), B(G)) \cong(\mu: M \rightarrow G)
$$

Teorema. Seja $X$ um $C W$-complexo reduzido, $Z$ um espaço conexo com $\pi_{2}(Z)=0$ e $f: Z \rightarrow X$ uma aplicação contínua que induz uma sobrejeção no grupo fundamental. Seja $\mu: M \rightarrow G$ o módulo cruzado fundamental da fibração associada a $f$. Então existe uma aplicação

$$
X \rightarrow B(\mu: M \rightarrow G)
$$

que induz um isomorfismo em $\pi_{1}$ e $\pi_{2}$

Nesse último teorema podemos por exemplo tomar $Z$ como o 1-esqueleto $X^{1}$ de $X$, tal que $M \cong$ $\pi_{2}\left(X ; X^{1}\right)$. Segue portanto pelo teorema de Whitehead que para todo 2-tipo homotópico $X$ feita para uma escolha apropriada de fibração temos que $X \rightarrow B \circ \Pi(X)$ é uma equivalência homotópica. Como a construção do módulo cruzado fundamental depende de uma escolha de fibração, isso sugere que os espaços de classificação de diferentes módulos cruzados podem ser homotópicos. Acontece que podemos definir algebricamente um conceito de quasi-isomorfismo entre módulos cruzados, que são os morfismos que induzem equivalências homotópicas fracas entre os espaços de classificação, e é possível definir uma localização da categoria dos módulos cruzados invertendo esses quasi-isomorfismos de forma que essa localização é equivalente à categoria homotópica dos 2-tipos homotópicos. É nesse sentido que dizemos que os módulos cruzados modelam os 2-tipos homotópicos.

O fato que os módulos cruzados generalizam grupos e que sua relação com 2-tipos homotópicos é análoga à relação de grupos com os 1-tipos homotópicos sugere que para cada $n \in \mathbb{N}$ existe uma generalização de grupos que modela os $(n+1)$-tipos homotópicos. No presente trabalho vamos nos focar em dois tipos de estruturas algébricas, os cat ${ }^{n}$-grupos e os $n$-cubos cruzados de grupos, que Ellis e Steiner provaram ser equivalentes [ES87], e que definiremos precisamente no primeiro capítulo. Os módulos cruzados são equivalentes a 1-cubos cruzados de grupos e os 0-cubos cruzados de grupos são grupos.

Assim como para associar um módulo cruzado a um 2-tipo homotópico precisamos escolher uma fibração adequada, para associar um 2-cubo cruzado de grupos a um 3-tipo homotópico precisaremos de um 2-cubo de fibrações, que é um diagrama de espaços indexados por pares de subconjuntos disjuntos de $\{1,2\}$

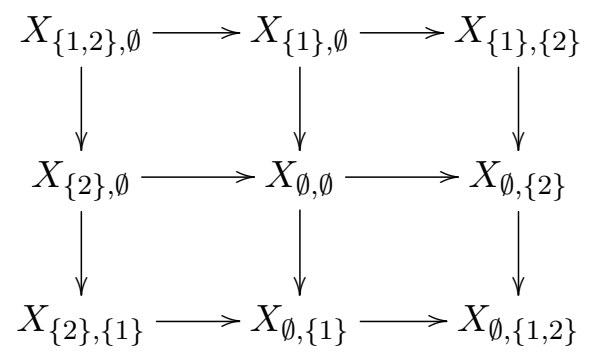

aonde $X_{A \cup\{i\}, B} \rightarrow X_{A, B} \rightarrow X_{A, B \cup\{i\}}$ são fibrações para $i \notin A \cup B$. O 2-cubo cruzado de grupos fundamental desse 2 -cubo de fibrações é então formado pelos grupos da forma $\pi_{1}\left(X_{A, \emptyset}\right)$. Por exemplo 
dados dois subespaços $U$ e $V$ de um espaço $X$ podemos obter o 2-cubo cruzado de grupos

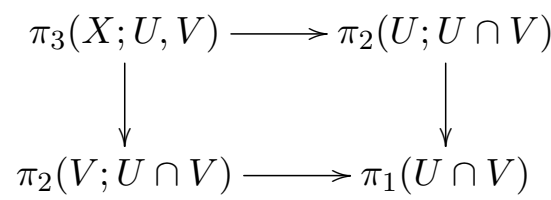

Assim como o módulo cruzado fundamental de uma fibração possui uma ação entre os grupos também temos emparelhamentos entre elementos dos grupos do 2-cubo cruzado de grupos fundamental na forma de um produto generalizado de Whitehead, que construiremos precisamente na seção 3.6, da forma $\otimes: \pi_{1}\left(X_{A, \emptyset}\right) \times \pi_{1}\left(X_{B, \emptyset}\right) \rightarrow \pi_{1}\left(X_{A \cup B, \emptyset}\right)$.

Um $n$-cubo cruzado de grupos é basicamente uma família de grupos $M_{A}$ indexados pelos subconjuntos do conjunto $\langle n\rangle=\{1,2, \ldots, n\}$, uma família de homomorfismos de grupos $\mu_{i}: M_{A} \rightarrow M_{A \backslash\{i\}}$ e uma família de funções $h: M_{A} \times M_{B} \rightarrow M_{A \cup B}$ que satisfazem certos axiomas. Como mencionamos antes a categoria dos $n$-cubos cruzados de grupos é equivalente à categoria dos cat ${ }^{n}$-grupos formada por grupos equipados com $2 n$ endomorfismos que satisfazem certos axiomas, e que está associado a teoria de $n$-categorias internas aos grupos. A vantagem de ter dois modelos algébricos equivalentes porém distintos é que algumas computações são mais naturais na categoria dos $n$-cubos cruzados de grupos, enquanto geralmente as demonstrações de teoremas são mais simples usando a categoria dos cat $^{n}$-grupos. Durante o trabalho intercambiaremos com frequência entre esses dois tipos de estruturas.

No caso geral associaremos a categoria de $n$-cubos de fibrações à categoria dos $(n+1)$-tipos homotópicos, e construiremos os funtores $n$-cubo cruzado de grupos fundamental, que denotamos por $\Pi$, e cat ${ }^{n}$-grupo fundamental, que denotamos por $\Pi$. Assim como no caso dos módulos cruzados segue do trabalho de Loday [Lod82] que existe um funtor espaço de classificação, denotado por $B$, que associa a cada $n$-cubos cruzados de grupos um CW-complexo com propriedades análogas ao funtor espaço de classificação dos módulos cruzados. Mostraremos como as propriedades dos funtores mencionados implicam que os $n$-cubos cruzados de grupos e os cat ${ }^{n}$-grupos modelam os $(n+1)$-tipos homotópicos da mesma forma que os módulos cruzados modelam os 2-tipos homotópicos.

\section{Generalização do teorema de Seifert-van Kampen}

Uma das principais ferramentas para computar o grupo fundamental de um espaço $X$ é o teorema de Seifert-van Kampen, que nos diz que se $X$ for a união de dois abertos conexos por caminhos $U_{1}$ e $U_{2}$ tal que a intersecção desses abertos é conexa por caminhos, então $X$ é conexo por caminhos e no diagrama de pushout abaixo o morfismo natural $k$ é um isomorfismo. Em outras palavras o funtor grupo fundamental preserva o diagrama de pushout nesse caso.

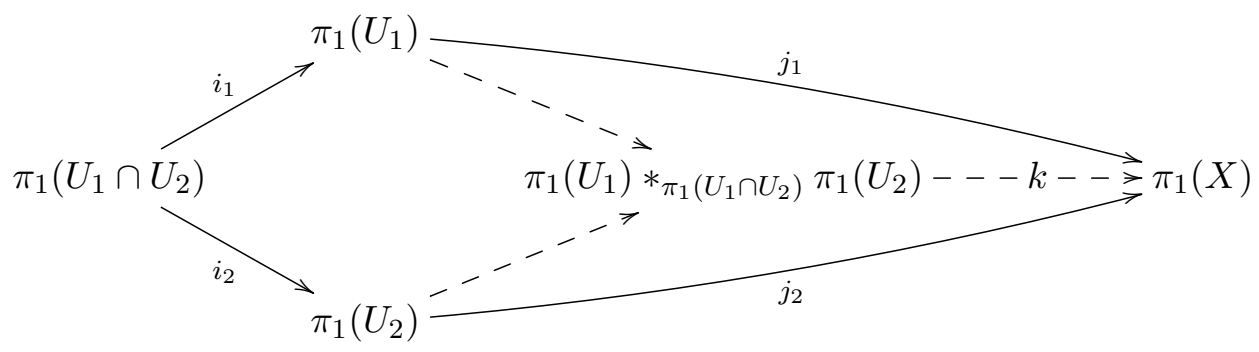

Esse resultado pode ser generalizado para um recobrimento por abertos arbitrário $U=\left\{U_{\lambda}\right\}_{\lambda \in \Lambda}$ 
tal que cada $U_{\lambda}$ contém o ponto base de $X$. Denote por $\Lambda_{\text {fin }}$ a categoria dos subconjuntos finitos não vazios de $\Lambda$, cujos morfismos são as inclusões, e por $U_{\sigma}=\underset{\lambda \in \sigma}{\cap} U_{\lambda}$ para $\sigma \in \Lambda_{\text {fin }}$. O teorema de Seifert-van Kampen para recobrimentos com um número arbitrário de abertos pode ser expresso da seguinte forma.

Teorema. Seja $X$ um espaço topológico e seja $\left\{U_{\lambda}\right\}_{\lambda \in \Lambda}$ um recobrimento por abertos de $X$ tal que todos os abertos contém o ponto base. Suponha que cada $U_{\sigma}$ é conexo por caminhos para todo $\sigma \in \Lambda_{\text {fin }}$. Então

(C) X é conexo por caminhos;

(I) o homomorfismo natural de espaços topológicos

$$
\underset{\sigma \in \Lambda_{f i n}}{\operatorname{colim}_{1}} \pi_{1}\left(U_{\sigma}\right) \rightarrow \pi_{1}(X)=\pi_{1}\left(\underset{\sigma \in \Lambda_{f i n}}{\operatorname{colim}} U_{\sigma}\right)
$$

é um isomorfismo.

Ou seja o funtor $\pi_{1}$ preserva o colimite de diagramas dados pelas hipóteses do teorema. Uma das dificuldades de computar os grupos de homotopia de dimensões maiores que 1 é que para esses funtores o teorema acima não vale. Brown e Loday provaram [BL87a] que o funtor $\Pi$ preserva colimites de certos diagramas aonde todos os espaços envolvidos são conexos por caminho.

Teorema (Teorema de Seifert-van Kampen para $n$-cubos fibrações). Seja X um n-cubo de fibrações e seja $\left\{U_{\lambda}\right\}_{\lambda \in \Lambda}$ um recobrimento por abertos de $X_{\emptyset,\langle n\rangle}$ tal que todos os abertos contém o ponto base. Cada $U_{\sigma}$ para $\sigma \in \Lambda_{\text {fin }}$ determina por imagem inversa um n-cubo de fibrações que também denotaremos por $U_{\sigma}$. Suponha que cada $U_{\sigma}$ é um n-cubo de fibrações conexo. Então

(C) o n-cubo de fibrações $X$ é conexo;

(I) o homomorfismo natural de cat $^{n}$-grupos

$$
\operatorname{colim}_{\sigma \in \Lambda_{f i n}} \Pi\left(U_{\sigma}\right) \rightarrow \Pi(X)=\Pi\left(\underset{\sigma \in \Lambda_{f i n}}{\operatorname{colim}} U_{\sigma}\right)
$$

é um isomorfismo.

\section{Aplicações}

O fato que o funtor $n$-cubo cruzado de grupos fundamental satisfaz o teorema generalizado de Seifert-van Kampen sugere que podemos computar o $n$-cubo cruzado de grupos fundamental de um $n$-cubo de fibrações usando técnicas análogas às usadas para computar o grupo fundamental de espaços.

Veremos como essa analogia nos permite generalizar certos resultados clássicos da topologia algébrica. Apresentaremos como Brown e Loday provaram em [BL87b] uma versão do teorema de Blakers-Massey com hipóteses enfraquecidas assim como uma generalização do teorema de $\mathrm{Hu}-$ rewicz. Mostramos também como esse último resultado foi usado por Brown e Ellis em [BE88] para apresentar uma fórmula para os grupos de homologia de um grupo análoga a fórmula de Hopf para o segundo grupo de homologia.

Apresentamos também como Ellis e Mikhailov utilizaram as ideias apresentadas para derivar em [EM10] uma apresentação dos grupos de homotopia da esfera $\mathbb{S}^{2}$ encontrada originalmente por Wu usando outros métodos[Wu01]. 


\section{Organização do trabalho}

O presente trabalho é uma apresentação da teoria subjacente aos resultados mencionados, conhecida como topologia algébrica não-abeliana. Apresentaremos as estruturas algébricas e mostraremos tanto como elas surgem naturalmente da teoria de categorias de dimensões altas e como fazer certos cálculos necessários para as aplicações que faremos depois. Mostraremos como essas estruturas se relacionam com a teoria de homotopia e daremos exemplos de aplicações dessa teoria.

No primeiro capítulo definimos as categorias dos $n$-cubos cruzados de grupos e dos cat ${ }^{n}$-grupos, e em seguida demonstramos a equivalência dessas categorias. A importância de apresentar ambas as categorias é que a estrutura dos cat ${ }^{n}$-grupos em muitos casos tornam as demonstrações de certos teoremas mais simples, enquanto costuma ser mais fácil fazer cálculos usando os $n$-cubos cruzados de grupos. Como o teorema central da teoria, o teorema de Seifert-van Kampem generalizado, afirma que certos funtores com imagem nessas categorias preservam certos colimites as próximas seções são dedicadas a estudar diferentes tipos de colimites de $n$-cubos cruzados de grupos.

No segundo capítulo apresentamos na primeira seção as categorias de $n$-cubos fibrantes e a de $n$-cubos de fibrações, e demonstramos a equivalência delas. O motivo de usar ambas as categorias é porque a estrutura de $n$-cubos fibrantes simplifica a demonstração de certos teoremas, enquanto a definição do funtor $n$-cubo cruzado de grupos fundamental é muito mais transparente nos $n$-cubos de fibrações. Na seção seguinte demonstramos a equivalência da localização de uma certa subcategoria dos $n$-cubos fibrantes, chamada de $n$-cubos de Eilenberg-MacLane, obtida invertendo equivalências de espaço base, e a categoria de $n$-tipos homotópicos conexos.

No terceiro capitulo estudamos a relação entre as estruturas algébricas do primeiro capítulo e as estruturas topológicas do segundo capítulo. A primeira seção consiste de preliminares de conceitos e resultados de homotopia simplicial que serão necessários no resto do capítulo. Na segunda seção definimos o funtor cat ${ }^{n}$-grupo fundamental $\Pi$ de um $n$-cubo fibrante. Na terceira seção apresentamos o funtor espaço de classificação $B$ de um cat $^{n}$-grupo, e também como criar a partir dele um funtor $n$-cubo de fibrações de classificação $\mathcal{B}$. Na terceira seção mostramos como os funtores $\Pi$ e $\mathcal{B}$ formam uma equivalência entre a categoria dos $n$-cubos de Eilenberg-MacLane e a categoria dos cat $^{n}$ grupos. Esse resultado, junto com a equivalência do capitulo anterior, mostra como essas estruturas algébricas de fato capturam toda informação homotópica até a dimensão $n+1$, e na quarta seção mostramos como obter os grupos de homotopia do espaço $B(G)$ associado a um cat $^{n}$-grupo $G$. Na quinta seção provamos o teorema de Seifert-van Kampem generalizado, que afirma que esse funtor preserva colimites de certos diagramas e que nesses casos conectividade é preservada. Na sexta seção damos uma apresentação direta do funtor $n$-cubo cruzado de grupos fundamental de um $n$-cubo de fibrações, denotado $\Pi$, cuja existência é garantida pela equivalência entre cat $^{n}$-grupos e $n$-cubos cruzados de grupos.

O quarto capítulo é dedicado a apresentar aplicações concretas dessa teoria. Na primeira seção demonstraremos o resultado de Whitehead [Whi49] que descreve o grupo de homotopia relativo $\pi_{2}(X ; A)$ para um espaço $X$ obtido colando 2-células em $A$ como o módulo cruzado livre gerado pelas funções de colagem das 2-células. Na segunda seção apresentamos o resultado de Brown e Loday [BL87b] de uma versão do teorema de Blakers-Massey sobre grupos de homotopia de tríade com as hipóteses enfraquecidas. Na terceira seção vemos como Brown e Loday no artigo [BL87b] obtiveram uma generalização do teorema de excisão homotópica e do teorema de Hurewicz, e como isso permitiu que em [BE88] Brown e Ellis encontrassem fórmulas para os grupos de homologia de 
grupos em dimensões arbitrárias análogas à fórmula de Hopf para o segundo grupo de homologia. Na quarta seção apresentamos como Ellis e Mikhailov derivaram em [EM10] a fórmula para os grupos de homotopia da esfera $\mathbb{S}^{2}$ encontrada originalmente por $\mathrm{Wu}[\mathrm{Wu} 01]$.

Assumimos que todos os espaços são espaços pontuados compactamente gerados e fracamente Hausdorff [May99, Cap. 5], e todos os morfismos entre espaços preservam os pontos base. 



\section{Capítulo 1}

\section{Generalizações de módulos cruzados}

A principal estrutura algébrica usada na presente teoria é a de $n$-cubos cruzados de grupos. Essa estrutura generaliza os módulos cruzados, que foram descritos e utilizados pela primeira vez por Whitehead para estudar pares de espaços $(X, A)$ pontuados como foi descrito na introdução [Whi41, Whi46, Whi49].

Um módulo cruzado é definido como um homomorfismo de grupos $\mu: M \rightarrow G$ junto com uma ação de $G$ em $M$ tal que

$$
\begin{gathered}
\mu\left({ }^{g} m\right)=g \mu(m) g^{-1} \\
m n m^{-1}={ }^{\mu(m)} n
\end{gathered}
$$

Exemplos algébricos de tal estrutura são:

- a inclusão de um subgrupo normal, com a ação dada por conjugação;

- a aplicação do automorfismo interno $\chi: G \rightarrow$ Aut $G$, em que $\chi(g)$ é o automorfismo $h \mapsto$ $g h g^{-1}$

- a aplicação trivial $M \rightarrow G$ aonde $M$ é um $G$-módulo;

- um epimorfismo $M \rightarrow G$ cujo kernel está contido no centro de $M$.

Note que pelos axiomas temos que para todo módulo cruzado $\mu: M \rightarrow G$ a imagem de $\mu$ é normal em $G$, que o kernel de $\mu$ é central em $M$ e que os elementos de Im $\mu$ agem trivialmente nos elementos de Ker $\mu$, de forma que Ker $\mu$ herda uma ação de $G / \operatorname{Im} \mu$.

Nesse capítulo definiremos as categorias dos $n$-cubos cruzados de grupos e a dos cat ${ }^{n}$-grupos, e demonstramos sua equivalência. Usaremos os cat ${ }^{n}$-grupos para provar os teoremas do capítulo $\mathbf{3}$, incluindo o teorema generalizado de Seifert-van Kampen, enquanto os $n$-cubos cruzados de grupos serão usados nas aplicações do capítulo 4, sendo que a troca de estrutura algébrica usada é justificada pela equivalência entre elas. Como preliminares para as aplicações, as últimas seções desse capítulo são reservadas para mostrar como computar certos colimites na categoria dos $n$-cubos cruzados de grupos.

\section{$1.1 \quad n$-Cubos cruzados de grupos}

A generalização do conceito de módulos cruzados para $n$-cubos cruzados de grupos pode ser pensado considerando grupos como 0-cubos cruzados e considerando $n$-cubos cruzados como um módulo cruzado de $(n-1)$-cubos cruzados. Damos a definição de um $n$-cubo cruzado de grupos dada em [ES87]. 
Definição 1.1.1. Um $n$-cubo cruzado $M$ é uma família de grupos $M_{A}$, para $A \subseteq\langle n\rangle$ mais homomorfismos $\mu_{i}: M_{A} \rightarrow M_{A \backslash\{i\}}$, para $i \in\langle n\rangle$ e $A \subseteq\langle n\rangle$ e funções $h: M_{A} \times M_{B} \rightarrow M_{A \cup B}$ para $A, B \subseteq\langle n\rangle$ tais que se ${ }^{a} b$ denota $h(a, b) b$ para $a \in M_{A}$ e $b \in M_{B}$ com $A \subseteq B$, então para $a, a^{\prime} \in M_{A}, b, b^{\prime} \in M_{B}, c \in M_{C}$ e para $i, j \in\langle n\rangle$,

$$
\begin{gathered}
\mu_{i} a=a \text { se } i \notin A ; \\
\mu_{i} \mu_{j} a=\mu_{j} \mu_{i} a ; \\
\mu_{i} h(a, b)=h\left(\mu_{i} a, \mu_{i} b\right) ; \\
h(a, b)=h\left(\mu_{i} a, b\right)=h\left(a, \mu_{i} b\right) \text { se } i \in A \text { e } i \in B ; \\
h\left(a, a^{\prime}\right)=\left[a, a^{\prime}\right] ; \\
h(a, b)=h(b, a)^{-1} ; \\
h(a, b)=1 \text { se } a=1 \text { ou } b=1 ; \\
h\left(a a^{\prime}, b\right)={ }^{a} h\left(a^{\prime}, b\right) h(a, b) ; \\
h\left(a, b b^{\prime}\right)=h(a, b)^{b} h\left(a, b^{\prime}\right) ; \\
{ }^{a} h\left(h\left(a^{-1}, b\right), c\right)^{c} h\left(h\left(c^{-1}, a\right), b\right)^{b} h\left(h\left(b^{-1}, c\right), a\right)=1 ; \\
{ }^{a} h(b, c)=h\left({ }^{a} b{ }^{a} c\right) \text { se } A \subseteq B \text { e } A \subseteq C .
\end{gathered}
$$

Note que ( $\left.{ }^{n} \mathrm{CG} 1\right)$ e ( $\left.\mathrm{I}^{n} \mathrm{CG} 2\right)$ equivalem a dizer que os $M_{A}$ e os $\mu_{i}$ formam um $n$-cubo comutativo de grupos, ou que eles definem um funtor do conjunto parcialmente ordenado dos subconjuntos de $\langle n\rangle$ na categorias de grupos. As equações ( $\left.{ }^{n} \mathrm{CG} 3\right)$ e ( $\left.{ }^{n} \mathrm{CG} 4\right)$ nos dão condições de compatibilidade das funções $h$ e $\mu$. Temos por ( ${ }^{n}$ CG5) que as funções $h$ são uma generalização de comutadores, e por ( $\left.\mathrm{I}^{n} \mathrm{CG} 6\right)-\left(\mathrm{I}^{n} \mathrm{CG} 11\right)$ temos que $h$ respeita relações usuais do comutador.

Note que por ( $\left.\mathrm{I}^{n} \mathrm{CG} 7\right)$-(I $\left.{ }^{n} \mathrm{CG} 9\right)$ se $A \subseteq B$ então a função $M_{A} \times M_{B} \rightarrow M_{B}$ dada por $(a, b) \mapsto{ }^{a} b$ define uma ação de $M_{A}$ em $M_{B}$.

Devido a ( $\left.{ }^{n} \mathrm{CG} 1\right)$ e ( $\left.\mathrm{I}^{n} \mathrm{CG} 4\right)-\left(\mathrm{I}^{n} \mathrm{CG} 6\right)$ muitos dos $\mu_{i}$ e $h$ são redundantes, em particular $\left(\mathrm{I}^{n} \mathrm{CG} 4\right)$ implica que todas as funções $h$ estão totalmente definida pelos casos em que $A$ e $B$ são disjuntos, porém a apresentação dos axiomas fica mais simples mantendo essas redundâncias.

No caso $n=1$ a definição pode ser simplificada e se ve que ela equivale a definição de módulo cruzado. Mais do que isso, para qualquer par de subconjuntos $A, A^{\prime} \in\langle n\rangle$ com $A^{\prime} \subset A$ temos que as aplicações $\underset{i \in A \backslash A^{\prime}}{\circ} \mu_{i}: M_{A} \rightarrow M_{A^{\prime}}$ junto com a ação de $M_{A^{\prime}}$ em $M_{A}$ formam um módulo cruzado.

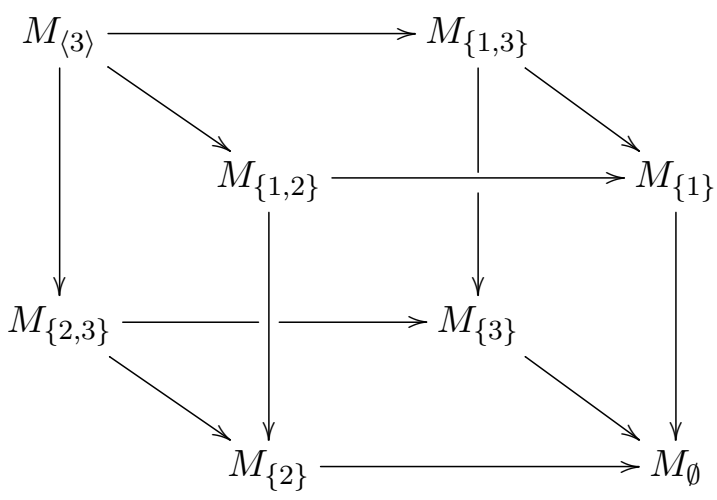

Figura 1.1: 3-cubo cruzado de grupos. 
Definição 1.1.2. Um morfismo de $n$-cubos cruzados $M \rightarrow N$ é uma família de homomorfismos de grupos $M_{A} \rightarrow N_{A}$ para $A \subseteq\langle n\rangle$ que comutam com as aplicações $\mu_{i}$ e $h$.

Um exemplo simples de um $n$-cubo cruzado de grupos pode ser construído a partir de um grupo $G$ e um conjunto $\left\{N_{i} \mid i \in\langle n\rangle\right\}$ de subgrupos normais de $G$. Definindo $M_{A}=\bigcap_{i \in A} N_{i}$ para $A \neq \emptyset$ e $M_{\emptyset}=G$, tomando os $\mu_{i}$ como inclusões e as funções $h$ como a função comutadora obtemos um $n$-cubo cruzado de grupos.

\subsection{Cat $^{n}$-grupos}

Para as demonstrações dos teoremas do capítulo $\mathbf{3}$ precisaremos de uma construção equivalente à da seção anterior, mas antes de definir essa construção veremos uma breve preliminar de conceitos categóricos.

Seja $\mathcal{C}$ uma categoria com pullbacks. Uma categoria interna a $\mathcal{C}$ é um par de objetos $C_{1}$ e $C_{0}$ de $\mathcal{C}$, denotados o objeto de morfismos e o objeto de objetos respectivamente, junto com os morfismos de $\mathcal{C}$

- $o, d: C_{1} \rightarrow C_{0}$ chamados morfismos de origem e destino respectivamente

- $i: C_{0} \rightarrow C_{1}$ chamado de morfismo de identidade

- $c: C_{1} \times_{C_{0}} C_{1} \rightarrow C_{1}$ chamado de morfismo de composição

aonde o domínio do terceiro morfismo é o pullback obtido de $o$ e $d$, tais que os seguintes diagramas comutem:

- leis especificando a origem e destino dos morfismos identidade:
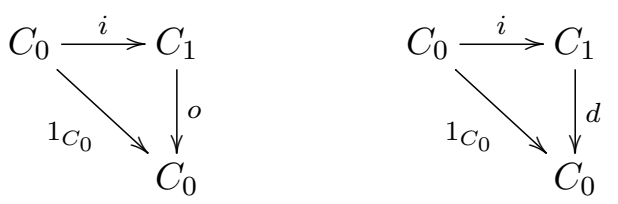

- leis especificando a origem e destino de morfismos compostos:
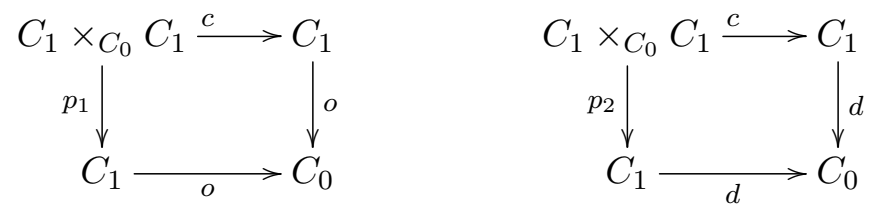

- a lei associativa da composição:

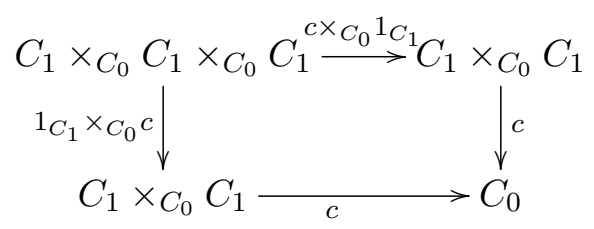

- as leis de unidade a esquerda e a direita para a composição:

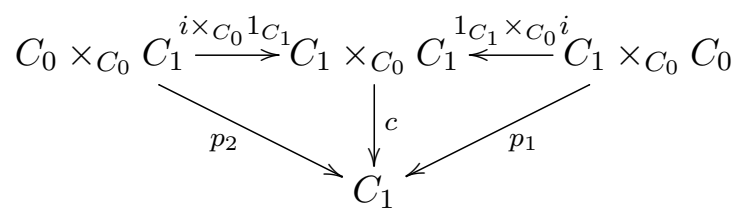


Um funtor interno a $\mathcal{C}$ é um par de morfismos $F_{1}: C_{1} \rightarrow C_{1}^{\prime}$ e $F_{0}: C_{0} \rightarrow C_{0}^{\prime}$ que comuta com todos os morfismos acima.

Iremos agora nos focar no caso das categorias internas a categoria dos grupos. Existe uma apresentação das informações contidas em uma categoria interna aos grupos, conhecida como um cat $^{1}$-grupo, que é definido como um grupo $G$ junto com dois endomorfismos $s, t: G \rightarrow G$ tais que

$$
\begin{aligned}
& s \circ t=t ; \\
& t \circ s=s ;
\end{aligned}
$$

$[$ ker $s$, ker $t]=1$.

Note que por esses axiomas $s$ e $t$ são projeções (possivelmente distintas) no mesmo subgrupo normal de $G$.

O cat $^{1}$-grupo associado a uma categoria interna aos grupos é dado por $G=G_{1}, s=i \circ o$ e $t=i \circ d$. A categoria interna aos grupos associada a um cat ${ }^{1}$-grupo $G$ é dado por

$$
\begin{gathered}
G_{0}=\operatorname{Im} s ; \\
G_{1}=\operatorname{Ker} s \rtimes \operatorname{Im} s \cong G ; \\
i(a)=(1, a) ; \\
o(k, a)=a ; \\
d(k, a)=t(k) a ; \\
c\left((k, a),\left(k^{\prime}, t(k) a\right)\right)=\left(a, k^{\prime} k\right) .
\end{gathered}
$$

Note que por $c$ ser um homomorfismo temos que $c\left(g_{1} h_{1}, g_{1}^{\prime} h_{1}^{\prime}\right)=c\left(g_{1}, g_{1}^{\prime}\right) c\left(h_{1}, h_{1}^{\prime}\right)$, o que é conhecido como lei do intercambio. Temos que a terceira equação que define um cat ${ }^{1}$-grupo é equivalente à lei do intercambio valer para a categoria interna aos grupos.

Podemos definir uma $n$-categoria interna a $\mathcal{C}$ como uma categoria interna a categoria de $(n-1)$ categorias internas a $\mathcal{C} .{ }^{1}$ Os cat $^{n}$-grupos que apresentaremos agora, introduzidos pela primeira vez em [Lod82] generalizam o conceito de cat $^{1}$-grupo e podem ser pensados como estruturas algébricas que encapsulam a informação em uma $n$-categoria interna a categoria de grupos.

Definição 1.2.1. Um cat ${ }^{n}$-grupos é um grupo $G$ equipado com $2 n$ endomorfismos $s_{i}, t_{i}(i \in\langle n\rangle)$ tais que para $i, j \in\langle n\rangle$ com $i \neq j$ temos

$$
\begin{aligned}
& t_{i} s_{i}=s_{i} ; \\
& s_{i} t_{i}=t_{i} ;
\end{aligned}
$$

$\left[\right.$ Ker $s_{i}$, Ker $\left.t_{i}\right]=1$;

$$
\begin{aligned}
& s_{i} s_{j}=s_{j} s_{i} ; \\
& t_{i} t_{j}=t_{j} t_{i} ; \\
& s_{i} t_{j}=t_{j} s_{i} .
\end{aligned}
$$

Definição 1.2.2. Um morfismo de cat ${ }^{n}$-grupos $G \rightarrow H$ é um homomorfismo de grupos que comuta com as aplicações $s$ e $t$.

\footnotetext{
${ }^{1}$ Mais precisamente estamos definindo um modelo para strict $n$-fold categories internal to $\mathcal{C}$.
} 
Em algumas ocasiões podemos ter um grupo e um conjunto de endomorfismos como na definição de um cat ${ }^{n}$-grupo, exceto que o axioma $\left(\mathrm{C}^{n} \mathrm{G} 3\right)$ não é necessariamente satisfeito.

Definição 1.2.3. um grupo $G$ equipado com $2 n$ endomorfismos $s_{i}, t_{i}(i \in\langle n\rangle)$ que satisfazem os axiomas de um cat $^{n}$-grupo mas não necessariamente o axioma $\left(\mathrm{C}^{n} \mathrm{G} 3\right)$ é um pré-cat ${ }^{n}$-grupo. Os morfismos nas categorias são homomorfismos de grupos que comutam com todos os endomorfismos.

Para todo pré-cat ${ }^{n}$-grupo podemos associar canonicamente um cat $^{n}$-grupo tomando o quociente pelos subgrupos [Ker $s_{i}$, Ker $\left.t_{i}\right](i \in\langle n\rangle)$ do grupo subjacente. Denotamos o funtor que faz essa associação por ass.

\subsection{Equivalência de $n$-cubos cruzados de grupos e cat $^{n}$-grupos}

Demonstraremos agora a equivalência das categorias apresentadas até agora. Esse resultado foi provado em [ES87] e seguiremos as mesmas ideias encontradas nesse artigo. Essa equivalência justifica a aplicação do teorema generalizado de Seifert-van Kampen ao funtor $n$-cubo cruzado fundamental, sendo que a demonstração usa o funtor cat ${ }^{n}$-grupo fundamental.

A ideia básica é que queremos decompor os cat ${ }^{n}$-grupos em grupos indexados pelos subconjuntos de $\langle n\rangle$ usando os endomorfismos da definição de forma que as funções $h$ sejam dadas pela função comutadora nos cat ${ }^{n}$-grupos. Por outro lado queremos também juntar os diferentes grupos de um $n$-cubo cruzado de grupos em um único grupo aonde haverão endomorfismos naturais que nos darão a estrutura de cat $^{n}$-grupos. Primeiro, alguns lemas preliminares que nos ajudarão no processo de criar um cat $^{n}$-grupo a partir de um $n$-cubo cruzado de grupos.

Lema 1.3.1. Suponha que $(M, h)$ é uma família de grupos $M_{A}$ indexados pelos subconjuntos de $\langle n\rangle$ e funções $h: M_{A} \times M_{B} \rightarrow M_{A \cup B}$ satisfazendo $\left(I^{n} C G 5\right)-\left(I^{n} C G 11\right)$. Seja $\Phi$ uma família de subconjuntos de $\langle n\rangle$ fechada sob união e seja

$$
\left.P_{\Phi}=\frac{*_{A \in \Phi} M_{A}}{h(a, b)=[a, b]} \text { para } a \in M_{A}, b \in M_{B} \operatorname{com} A, B \in \Phi\right) .
$$

Então para alguma ordem total de $\Phi$, a função multiplicativa

$$
\begin{aligned}
\prod_{A \in \Phi} M_{A} & \rightarrow P_{\Phi} \\
\left(x_{A}\right)_{A \in \Phi} & \mapsto \prod_{A \in \Phi} x_{A}
\end{aligned}
$$

é bijetora.

Demonstração: Provamos por indução no tamanho de $\Phi$, sendo que o caso $\Phi=\emptyset$ é trivial. Se $\Phi$ é não-vazio, então seja $A$ o membro mínimo de $\Phi$ e seja $\Psi=\Phi \backslash\{A\}$. A hipotese de indução é aplicavel a $\Psi$, logo basta provar que $P_{\Phi}$ é um semi-produto direto de $M_{A}$ e $P_{\Psi}$. Obtemos $P_{\Phi}$ de $M_{A} * P_{\Psi}$ impondo a relação

$$
h(a, b)=[a, b] \text { para } b \in M_{B}, c \in M_{C} \operatorname{com} B, C \in \Phi, \text { aonde } B=A \text { ou } C=A .
$$

A relação com $B=C=A$ já vale em $M_{A}$ por ( ${ }^{n} \mathrm{CG}$ ), e a relação com $B=A$ são equivalentes a aquelas com $C=A$, por ( $\left.\mathrm{I}^{n} \mathrm{CG} 6\right)$. Então as únicas relações necessárias são $h(a, b)=[a, b]$ para $a \in M_{A}, b \in M_{B}, B \in \Psi$. Como $h(a, b)=[a, b]$ é equivalente à $h(a, b) b=a b a^{-1}$ basta mostrar que as funções 


$$
(a, b) \mapsto h(a, b) b: M_{A} \times M_{B} \rightarrow P_{\Psi}, B \in \Psi
$$

determinam uma ação de $M_{A}$ em $P_{\Psi}$.

Note que por (I ${ }^{n}$ CG9), fixado $a \in M_{A}$, a função $b \mapsto h(a, b) b: M_{B} \rightarrow P_{\Psi}$ é um homomorfismo. Esses homomorfismos determinam um endomorfismo $\gamma(a)$ de $P_{\Psi}$ devido ao seguinte lema, que será demonstrado a seguir.

Lema 1.3.2. Para $a \in M_{A}, b \in M_{B}, c \in M_{C} \operatorname{com} B, C \in \Psi$,

$$
h(a, h(b, c)) h(b, c)=[h(a, b) b, h(a, c) c]
$$

em $P_{\Psi}$.

Além disso a função $\gamma: M_{A} \rightarrow \operatorname{End} P_{\Psi}$ é um homomorfismo com imagem em Aut $P_{\Psi}$, ou seja é uma ação de $M_{A}$ em $P_{\Psi}$, devido a ( $\left.{ }^{n} \mathrm{CG} 7\right)$ e ( $\left.{ }^{n} \mathrm{CG} 8\right)$. Isso completa a demonstração do lema 1.3.1 assumindo lema 1.3.2.

Demonstração de 1.3.2: Precisaremos de três identidades em $P_{\Psi}$. Primeiro, como

$$
e \mapsto h(a, e) e: M_{E} \rightarrow P_{\Psi}
$$

é um homomorfismo para $E \in \Psi$, por (I $\left.{ }^{n} \mathrm{CG} 9\right)$,

$$
(h(a, e) e)^{-1}=h\left(a, e^{-1}\right) e^{-1} \text { para } e \in M_{E}, E \in \Psi .
$$

Consequentemente,

$$
e h\left(e^{-1}, a\right)=h(a, e) e \text { para } e \in M_{E}, E \in \Psi .
$$

pois ( $\left.\mathrm{I}^{n} \mathrm{CG} 6\right)$ nos dá $e h\left(e^{-1}, a\right)=e h\left(a, e^{-1}\right)^{-1}=\left(h\left(a, e^{-1}\right) e^{-1}\right)^{-1}$, que é equivalente à $h(a, e) e$ pelo lema 1.1. Finalmente,

$$
b h(a, d) d=h(a, b) b \text { aonde } d=h\left(a^{-1}, b^{-1}\right),
$$

pois

$$
\begin{aligned}
b h(a, d) d & =b^{a} d \\
& =b^{a} h\left(a^{-1}, b^{-1}\right) \\
& =b h\left(a a^{-1}, b^{-1}\right) h\left(a, b^{-1}\right)^{-1} \text { por }\left(\mathrm{I}^{n} \mathrm{CG} 8\right) \\
& =b h\left(b^{-1}, a\right) \text { por }\left(\mathrm{I}^{n} \mathrm{CG} 7\right) \text { e }\left(\mathrm{I}^{n} \mathrm{CG} 6\right) \\
& =h(a, b) b \text { por }(1.2) .
\end{aligned}
$$

Agora provamos o lema 1.3.2 em três casos especiais.

Caso 1: $A \subseteq B$ e $A \subseteq C$. Aqui o lema 1.3.2 se reduz a (I ${ }^{n} \mathrm{CG} 11$ ). 
Caso 2: $A \subseteq C$ e $B$ arbitrário. Este caso segue do caso 1:

$$
\begin{aligned}
h(a, h(b, c)) h(b, c) & =h(h(b, c), a)^{-1} b c b^{-1} c^{-1} \text { por }\left(\mathrm{I}^{n} \mathrm{CG} 6\right) \text { e as relações de } P_{\Psi} \\
& =b\left(^{b^{-1}} h(h(b, c), a)\right)^{-1} c b^{-1} c^{-1} \\
& =b^{a} h\left(h\left(a^{-1}, b^{-1}\right), c\right)^{c} h\left(h\left(c^{-1}, a\right), b^{-1}\right) c b^{-1} c^{-1} \text { por }\left(\mathrm{I}^{n} \mathrm{CG} 10\right) \\
& =b^{a} h(d, c) c h\left(c^{-1}, a\right) b^{-1} h\left(c^{-1}, a\right)^{-1} c^{-1} \text { aonde } d=h\left(a^{-1}, b^{-1}\right) \\
& =b h(a, h(d, c)) h(d, c) h(a, c) c b^{-1} h\left(a, c^{-1}\right) c^{-1} \text { por }(1.2) \text { e }\left(\mathrm{I}^{n} \mathrm{CG} 6\right) \\
& =b[h(a, d) d, h(a, c) c] h(a, c) c b^{-1} h\left(a, c^{-1}\right) c^{-1} \text { pelo caso 1, já que } d \in M_{A \cup B} \text { e } A \subseteq A \cup B, \\
& =[b h(a, d) d, h(a, c) c] \text { por }(1.1) \\
& =[h(a, b) b, h(a, c) c] \text { por }(1.3)
\end{aligned}
$$

Caso 3: $A \subseteq B$ e $C$ arbitrário. Este caso segue do caso 2 invertendo os dois lados; note que por (1.1) e ( ( $\left.{ }^{n} \mathrm{CG} 6\right)$ o inverso de $h(a, h(b, c)) h(b, c)$ é $h(a, h(c, b)) h(c, b)$.

O lema 1.3.2 segue do caso 3 da mesma forma que o caso 2 segue do caso 1 . Isso completa a demonstração.

A demonstração da equivalência entre as categorias de $n$-cubos cruzados e cat $^{n}$-grupos segue dos teoremas a seguir. O teorema 1.3.3 nos dará o método de como decompor os cat $^{n}$-grupos, enquanto o teorema 1.3.4 nos dará como juntar os grupos em um $n$-cubo cruzado de grupos de forma a formar um $\mathrm{cat}^{n}$-grupo.

Teorema 1.3.3. Seja $G$ um grupo com $n$ projeções comutantes $s_{1}, \ldots, s_{n}$. Para $A \subseteq\langle n\rangle$ seja

$$
M_{A}=\bigcap_{i \in A} \operatorname{Ker} s_{i} \cap \bigcap_{i \notin A} \operatorname{Im} s_{i}
$$

e para $A, B \in\langle n\rangle$ seja $h: M_{A} \times M_{B} \rightarrow M_{A \cup B}$ a restrição da função comutadora em $G$. Então

i) para toda ordem total dos subconjuntos de $\langle n\rangle$ a função multiplicativa

$$
\begin{aligned}
\prod_{A \subseteq\langle n\rangle} M_{A} & \rightarrow G \\
\left(x_{A}\right)_{A \subseteq\langle n\rangle} & \mapsto \prod_{A \subseteq\langle n\rangle} x_{A}
\end{aligned}
$$

é bijetora;

ii) $G$ pode ser obtido do produto livre dos $M_{A}$ impondo as relações $h(a, b)=[a, b]\left(a \in M_{A}, b \in\right.$ $\left.M_{B}, A, B \subseteq\langle n\rangle\right)$ :

$$
G=\frac{*_{A \subseteq\langle n\rangle} M_{A}}{h(a, b)=[a, b]} .
$$

Teorema 1.3.4. Sejam $M_{A}$, para $A \subseteq\langle n\rangle$, uma família de grupos e $h: M_{A} \times M_{B} \rightarrow M_{A \cup B}$, para $A, B \subseteq\langle n\rangle$, uma família de funções. Então as seguintes afirmações são equivalentes:

i) Existe um grupo $G$ com $n$ projeções comutantes $s_{1}, \ldots, s_{n}$ tal que

$$
M_{A}=\bigcap_{i \in A} \operatorname{Ker} s_{i} \cap \bigcap_{i \notin A} \operatorname{Im} s_{i} \text { para } A \subseteq\langle n\rangle
$$

e os $h$ são restrições da função comutadora de $G$; 
ii) Valem as equações ( $\left.{ }^{n} C G 5\right)-\left(I^{n} C G 11\right)$.

Demonstração de 1.3.3.i: Seja $x \in G$. Precisamos mostrar que $x=\Pi_{A \subseteq\langle n\rangle} x_{A}$ para uma escolha única de $x_{A} \in M_{A}$. Para unicidade, suponha que $x=\Pi_{A \subseteq\langle n\rangle} x_{A} \operatorname{com} x_{A} \in M_{A}$. Então

$$
\left(\circ_{j \notin A} s_{j}\right) x=\Pi_{B \subseteq A} x_{B}
$$

que pode ser resolvida em $x_{A}$ em termos de $x$ e dos $x_{B}$ para os quais $B$ é subconjunto de $A$. Logo, indutivamente, $x$ determina os $x_{A}$.

Para existência, definimos indutivamente os elementos $x_{A}$ de $G$ por (1.4). Fixando $A=\langle n\rangle$ nos dá $x=\Pi_{B \subseteq\langle n\rangle} x_{B}$, logo falta mostrar que $x_{A} \in M_{A}$. Faremos isso por indução no tamanho de $A$. Para $i \in A$, aplicar $s_{i}$ em (1.4) e depois usar (1.4) com $A$ substituído por $A \backslash\{i\}$ nos dá

$$
\Pi_{B \subseteq A} s_{i} x_{B}=\left(\circ_{j \notin A \backslash\{i\}} s_{j}\right) x=\Pi_{B \subseteq A \backslash\{i\}} x_{B} .
$$

Agora a hipótese de indução nos diz que se $B$ é um subconjunto próprio de $A$, então $s_{i} x_{B}=x_{B}$ para $B \subseteq A \backslash\{i\}$, e $s_{i} x_{B}=1$ caso contrario. Segue que $s_{i} x_{A}=1, \operatorname{logo} x_{A} \in \operatorname{Ker} s_{i}$. Para $i \notin A$ a hipótese de indução e (1.4) nos dá imediatamente que $x_{A} \in \operatorname{Im} s_{i}$. Logo

$$
x_{A} \in \bigcap_{i \in A} \operatorname{Ker} s_{i} \cap \bigcap_{i \notin A} \operatorname{Im} s_{i}=M_{A},
$$

como precisávamos. Isso completa a demonstração do teorema 1.3.3.i.

Demonstração de 1.3.4.i $\Rightarrow$ 1.3.4.ii: Devido a $h$ ser definido como a restrição da função comutadora de $G$ ( $\left.\mathrm{I}^{n} \mathrm{CG} 5\right)$ segue imediatamente, e como interpretando $h$ como a função comutadora as equações ( $\left.I^{n} \mathrm{CG}\right)-\left(I^{n} \mathrm{CG} 11\right)$ são relações usuais de comutadores a implicação segue automaticamente.

Demonstração de 1.3.3.ii: Seja $P=P_{\wp(\langle n\rangle)}$ como no lema 1.3.1. Como 1.3.4.i $\Rightarrow$ 1.3.4.ii podemos aplicar o lema 1.3.1 a $\left(M_{A}, h\right)$. Então o lema 1.3.1 e o teorema 1.3.3.i juntos implicam que o homomorfismo óbvio $P \rightarrow G$ é bijetor, que é o que queríamos

Demonstração de 1.3.4.ii $\Rightarrow$ 1.3.4.i: Seja $P=P_{\wp(\langle n\rangle)}$ como antes. Podemos definir projeções comutantes $s_{i}$ em $G$ da seguinte maneira: se $a \in M_{A}$, então

$$
s_{i} a= \begin{cases}1 & , i \in A, \\ a & , i \notin A .\end{cases}
$$

Os homomorfismos canônicos $M_{A} \rightarrow G$ podem ser restritos para nos dar homomorfismos

$$
M_{A} \rightarrow \bigcap_{i \in A} \operatorname{Ker} s_{i} \cap \bigcap_{i \notin A} \operatorname{Im} s_{i} .
$$

Pelo lema 1.3.1 e o teorema 1.3.3.ii esses devem ser isomorfismos. isso completa a demonstração.

Provaremos agora como os resultados acima de fato nos dão uma equivalência de categorias.

Teorema 1.3.5. As categorias dos cat ${ }^{n}$-grupos e dos n-cubos cruzados de grupos são equivalentes.

Demonstração: Pelo teorema 1.3.4 um grupo $G$ com $n$ projeções comutantes é equivalente à uma família de pares $\left(M_{A}, h\right)$ satisfazendo ( $\left.\mathrm{I}^{n} \mathrm{CG} 5\right)$-( $\left.\mathrm{I}^{n} \mathrm{CG} 11\right)$. A equivalência é dada por

$$
\begin{gathered}
M_{A}=\bigcap_{i \in A} \operatorname{Ker} s_{i} \cap \bigcap_{i \notin A} \operatorname{Im} s_{i}, \\
h(a, b)=[a, b] \text { para } a \in M_{A}, b \in M_{B}, \\
G=\frac{*_{A \subseteq\langle n\rangle} M_{A}}{h(a, b)=[a, b]},
\end{gathered}
$$


aonde a última equação vem do teorema 1.3.3.ii. Basta então mostrar que endomorfismos $t_{i}$ de $G$ fazendo de $\left(G, s_{i}, t_{i}\right)$ um cat $^{n}$-grupo são equivalentes a homomorfismos $\mu_{i}: M_{A} \rightarrow M_{A \backslash\{i\}}$ satisfazendo ( $\left.{ }^{n} \mathrm{CG} 1\right)-\left(\mathrm{I}^{n} \mathrm{CG} 4\right)$.

Note que para $a \in M_{A}$,

$$
s_{i} a= \begin{cases}1 & , i \in A, \\ a & , i \notin A .\end{cases}
$$

já que $s_{i} s_{i}=s_{i}$. Definindo $\mu_{i}: M_{A} \rightarrow M_{A \backslash\{i\}}$ como a restrição de $t_{i}$ a $M_{A}$ temos que $s_{i} t_{i}=s_{i}$ e $s_{j} t_{i}=t_{i} s_{j}$ para $i \neq j$ é equivalente à ( $\left.{ }^{n} \mathrm{CG} 3\right)$, que $t_{i} s_{i}=s_{i}$ equivale a ( $\left.{ }^{n} \mathrm{CG} 1\right)$ e que $t_{i} t_{j}=t_{j} t_{i}$ é equivalente à $\left(\mathrm{I}^{n} \mathrm{CG} 2\right)$. Falta mostrar que $\left[\operatorname{Ker} s_{i}, \operatorname{Ker} t_{i}\right]=1$ é equivalente à ( $\left.\mathrm{I}^{n} \mathrm{CG} 4\right)$.

Para isso usamos o teorema 1.3.3.ii. Fixando $i$, ordene os subconjuntos de $\langle n\rangle$ de forma que se $i \in A$, então $A$ precede imediatamente $A \backslash\{i\}$. Logo torna-se aparente que Ker $s_{i}$ é gerado pelos elementos $a \in M_{A}(i \in A)$ e que Ker $t_{i}$ é gerado pelos elementos $b^{-1}\left(t_{i} b\right)\left(b \in M_{B}, i \in B\right)$. Logo $\left[\right.$ Ker $s_{i}$, Ker $\left.t_{i}\right]=1$ é equivalente à $\left[a, b^{-1}\left(t_{i} b\right)\right]=1$ para $a \in M_{A}, b \in M_{B}, i \in A, i \in B$. Como

$$
\left[a, b^{-1}\left(t_{i} b\right)\right]=b^{-1}[a, b]^{-1}\left[a, t_{i} b\right] b,
$$

isso é equivalente à $h(a, b)=h\left(a \mu_{i} b\right)$ para $a \in M_{A}, b \in M_{B}, i \in A, i \in B$, que é uma metade de $\left(\mathrm{I}^{n} \mathrm{CG} 4\right)$. Como a outra metade é redundante dado ( $\left.{ }^{n} \mathrm{CG} 6\right)$ isso completa a demonstração.

\subsection{Resultados categoriais}

As próximas seções se focarão em descrever os colimites de certos diagramas na categoria de $n$ cubos cruzados de grupos, mas para isso será útil provar que uma certa família de funtores descrita abaixo preserva colimites.

Definição 1.4.1. Para cada $i \in\langle n\rangle$ existem os funtores $\check{\partial}_{i}^{+}$da categoria dos $n$-cubos cruzados de grupos a categoria dos $(n-1)$-cubos cruzados de grupos e os funtores $d_{i}$ da categoria dos $(n-1)$ cubos cruzados de grupos a categoria dos $n$-cubos cruzados de grupos tais que para todo $n$-cubo cruzado de grupos $M, A^{\prime} \subset\langle n-1\rangle$ e $i \in\langle n\rangle$ temos

$$
\left(\Im_{i}^{+}(M)\right)_{A^{\prime}}=M_{\widetilde{\partial}_{i}^{+}\left(A^{\prime}\right)} \text { aonde } \widetilde{ठ}_{i}^{+}\left(A^{\prime}\right)=\bigcup_{\substack{j \in A^{\prime} \\ j<i}}\{j\} \cup \bigcup_{\substack{j \in A^{\prime} \\ j \geq i}}\{j+1\}
$$

e para todo $(n-1)$-cubos cruzado $N, A \subseteq\langle n\rangle$ e $i \in\langle n\rangle$ temos

$$
\left(d_{i}(N)\right)_{A}=N_{d_{i} A} \text { aonde } d_{i} A=\bigcup_{\substack{j \in A \backslash\{i\} \\ j<i}}\{j\} \cup \bigcup_{\substack{j \in A \backslash\{i\} \\ j>i}}^{\bigcup}\{j-1\} .
$$

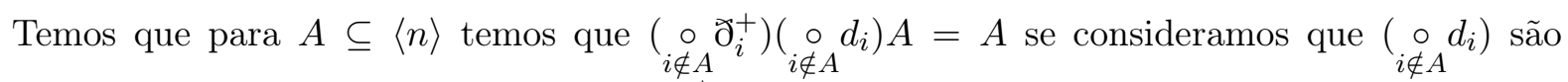
aplicados do maior $i$ para o menor e os $\left(\underset{i \notin A}{\circ} \int_{i}^{+}\right)$do menor $i$ para o maior. 


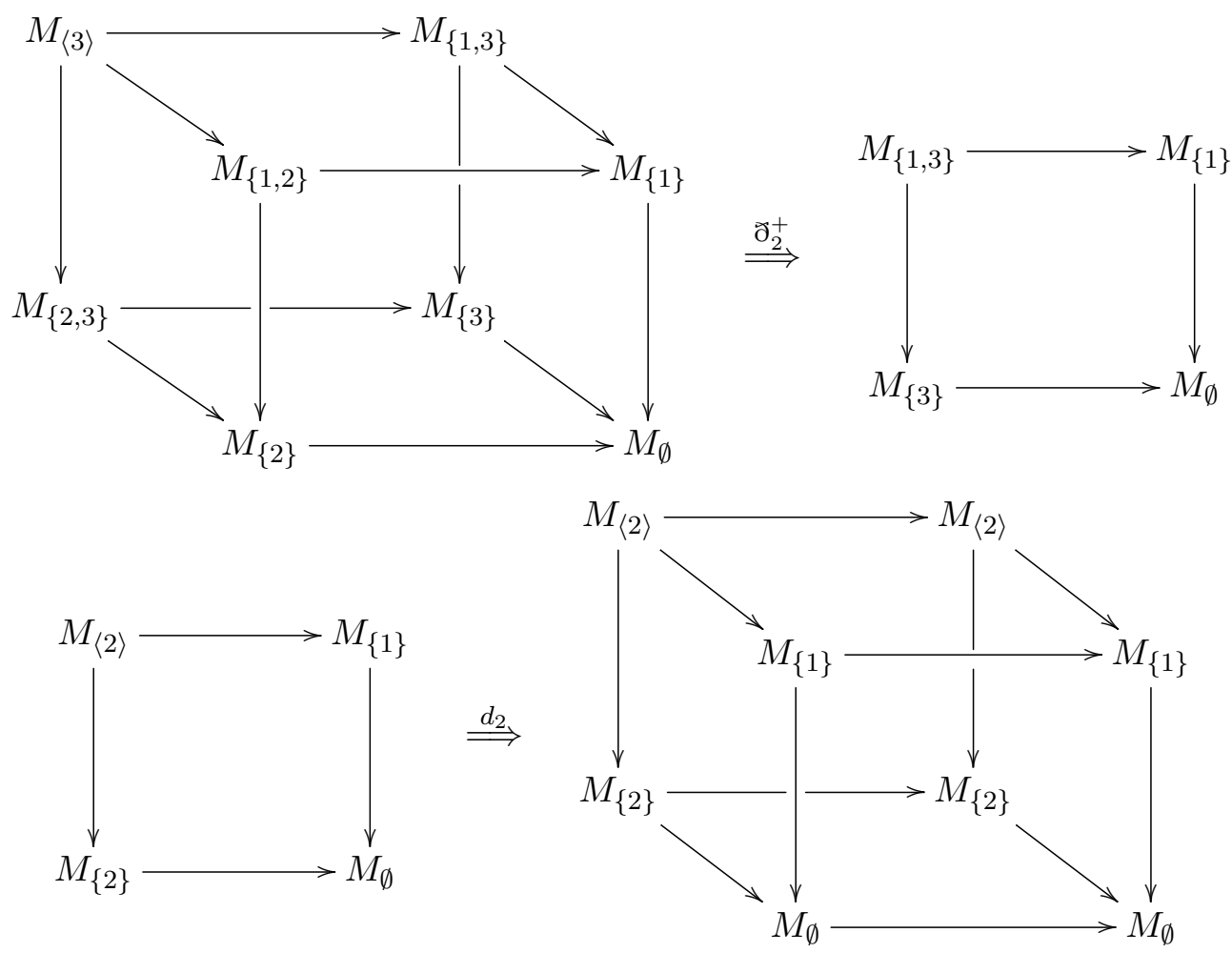

Figura 1.2: Funtores $\check{\mathrm{d}}_{2}^{+}$e $d_{2}$ no caso $n=3$.

Temos então o seguinte teorema, que tem como corolário o resultado que queríamos. A definição de adjunção pode ser encontrada no livro de Mac lane [Mac98].

Teorema 1.4.2. Para todo $i \in\langle n\rangle$ temos que $\tilde{\partial}_{i}^{+}$é adjunto a esquerda de $d_{i}$.

Demonstração: Note que $弓_{i}^{+} \circ d_{i}=\mathbb{1}_{n-1}$. Podemos tomar como a transformação natural

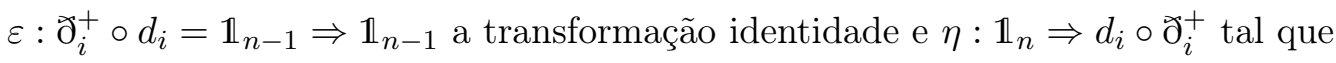

$$
\eta_{M_{A}}=\left\{\begin{array}{ll}
\mu_{i} & \text { se } i \in A \\
\mathbb{1}_{M_{A}} & \text { se } i \notin A
\end{array} .\right.
$$

Temos então que $\partial_{i}^{+}\left(\eta_{M_{A}}\right)=\mathbb{1}_{\check{\partial}_{i}^{+}\left(M_{A}\right)}$ e $\eta_{d_{i}\left(N_{A^{\prime}}\right)}=\mathbb{1}_{d_{i}\left(N_{A^{\prime}}\right)}$ e portanto

$$
\begin{aligned}
1_{{ठ_{i}^{+}}_{i}^{+}} & =\varepsilon \widetilde{ठ}_{i}^{+} \circ \Im_{i}^{+} \eta \\
1_{d_{i}} & =d_{i} \varepsilon \circ \eta d_{i},
\end{aligned}
$$

o que nos dá a adjunção.

Corolario 1.4.3. Os funtores $\partial_{i}^{+}$preservam colimites.

Demonstração: O corolário segue do fato que funtores adjuntos a esquerda preservam colimites [Mac98, Teo. V.5.1].

\subsection{Módulos cruzados induzidos}

Vamos analisar com mais detalhe o caso do 1-cubo cruzado de grupos, que como mencionado antes é equivalente à definição de módulo cruzado estudado por Whitehead. 
Definição 1.5.1. Um módulo cruzado $M$ é composto por dois grupos $M_{\{1\}}$ e $M_{\emptyset}$, uma ação de $M_{\emptyset}$ em $M_{\{1\}}$ e um homomorfismo $\mu: M_{\{1\}} \rightarrow M_{\emptyset}$ tais que para todos $m, m^{\prime} \in M_{\{1\}}$ e $n \in M_{\emptyset}$ temos

$$
\begin{aligned}
& \mu\left({ }^{n} m\right)=n \mu(m) n^{-1} \\
& m^{\prime} m m^{-1}={ }^{\mu\left(m^{\prime}\right)} m
\end{aligned}
$$

Definição 1.5.2. Um morfismo de módulos cruzados é um par de homomorfismos de grupos $f_{*}$ : $M_{*} \rightarrow N_{*}$ tais que o diagrama abaixo comuta

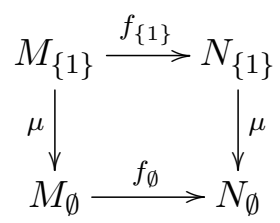

e que são compatíveis com as ações dos grupos no sentido que

$$
f_{\{1\}}\left({ }^{n} m\right)={ }^{f_{\emptyset}}(n) f_{\{1\}}(m) .
$$

Um conceito importante introduzido pela primeira vez em [BH78] é o de módulo cruzado induzido. Como veremos essa estrutura pode ser obtida como um certo tipo de pushout, que é um colimite, o que sugere a sua importância dado o teorema generalizado de Seifert-van Kampen. Veremos agora a definição desse conceito e alguns resultados que indicam como computar essa estrutura.

Definição 1.5.3. Seja $M$ um módulo cruzado, $G$ um grupo e $f_{\emptyset}: M_{\emptyset} \rightarrow G$ um homomorfismo de grupos. O módulo cruzado induzido de $M$ por $f_{\emptyset}$, denotado por $f_{\emptyset_{*}} M$, é definido pela propriedade universal que $\left(f_{\emptyset_{*}} M\right)_{\emptyset}=G$ e existe um morfismo de módulos cruzados $f: M \rightarrow f_{\emptyset *} M$ que estende $f_{\emptyset}$, ou seja é $f_{\emptyset}$ no índice $\emptyset$, e tal que para todo módulo cruzado $L$ com $L_{\emptyset}=G$ e morfismo de módulos cruzados $\phi: M \rightarrow L$ com $\phi_{\emptyset}=f_{\emptyset}$ existe um único morfismo de módulos cruzados $\psi: f_{*} M \rightarrow L$ tal que $\psi \circ f=\phi$

O teorema abaixo dá uma caracterização do módulo cruzado induzido como um pushout de módulos cruzados.

Teorema 1.5.4. Seja $f: M \rightarrow N$ um morfismo de módulos cruzados. Então $N$ é o módulo cruzado induzido de $M$ por $f_{\emptyset}$ se, e somente se, o diagrama de módulos cruzados abaixo for um pushout.

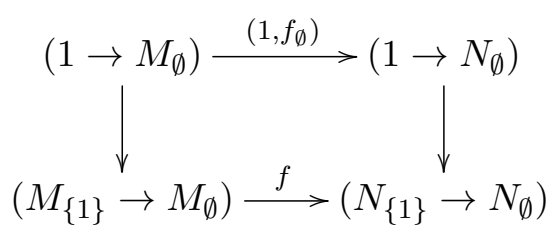


Demonstração: Note que para $N$ o pushout de um diagrama do tipo

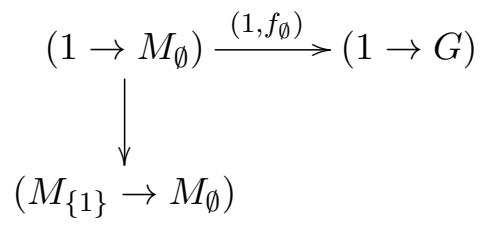

temos que $N_{\emptyset}=G$ como consequência do corolário 1.4.3.

Portanto esse teorema segue da observação que a composição dos morfismos tracejados no diagrama abaixo deve ser a identidade.

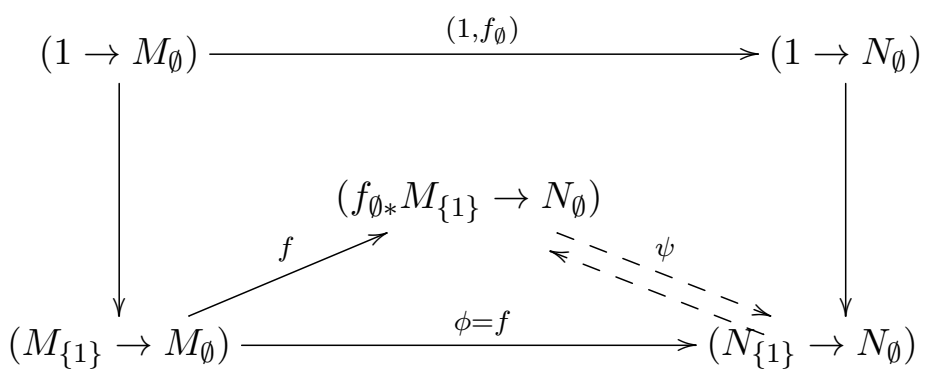

A existência dos módulos cruzados induzidos é garantida pela apresentação abaixo.

Teorema 1.5.5. Seja $M$ um módulo cruzado, $G$ um grupo e $f_{\emptyset}: M_{\emptyset} \rightarrow G$ um homomorfismo de grupos. então $f_{\emptyset *} M_{\{1\}}$ é gerado por $G \times M_{\{1\}}$ com as relações

$$
\begin{gathered}
(g, m)\left(g, m^{\prime}\right)=\left(g, m m^{\prime}\right) ; \\
\left(g,{ }^{n} m\right)=\left(g f_{\emptyset}(n), m\right) ; \\
\left(g^{\prime}, m^{\prime}\right)(g, m)\left(g^{\prime}, m^{\prime}\right)^{-1}=\left(g^{\prime} f_{\emptyset} \circ \mu\left(m^{\prime}\right) g^{\prime-1} g, m\right) .
\end{gathered}
$$

$O$ morfismo $\mu: f_{\emptyset *} M_{\{1\}} \rightarrow G$ é dado por $\mu((g, m))=g f_{\emptyset} \circ \mu(m) g^{-1}$ e a ação de $G$ é dada por $h(g, m)=(h g, m)$. O homomorfismo canônico $f_{\{1\}}: M_{\{1\}} \rightarrow f_{\emptyset_{*}} M_{\{1\}}$ é dado por $f_{\{1\}}(m)=(1, m)$.

Demonstração: Temos que $f_{*} M$ satisfaz os axiomas de módulos cruzados,

$$
\begin{aligned}
& \mu\left({ }^{h}(g, m)\right)=\mu((h g, m))=h g f_{\emptyset} \circ \mu(m) g^{-1} h^{-1}=h \mu((g, m)) h^{-1} \\
& \left(g^{\prime}, m^{\prime}\right)(g, m)\left(g^{\prime}, m^{\prime}\right)^{-1}=\left(g^{\prime} f_{\emptyset} \circ \mu\left(m^{\prime}\right) g^{\prime-1} g, m\right)=g^{\prime} f_{\emptyset} \circ \mu\left(m^{\prime}\right) g^{\prime-1}(g, m)=\mu\left(\left(g^{\prime}, m^{\prime}\right)\right)(g, m)
\end{aligned}
$$

e além disso

$$
\begin{gathered}
f_{\{1\}}\left({ }^{n} m\right)=\left(1,{ }^{n} m\right)=\left(f_{\emptyset}(n), m\right)={ }^{f_{\emptyset}(n)}(1, m)={ }^{f \emptyset(n)} f_{\{1\}}(m) \\
\mu \circ f_{*}(m)=\mu((1, m))=f \circ \mu(m)
\end{gathered}
$$

$\operatorname{logo} f=\left(f_{\{1\}}, f_{\emptyset}\right)$ é um morfismo de módulos cruzados.

Seja $\varphi: M \rightarrow N$ um morfismo de módulos cruzados com $N_{\emptyset}=G$ tal que $\varphi_{\emptyset}=f_{\emptyset}$, então podemos definir $\psi: f_{\emptyset *} M \rightarrow N$ com $\psi_{\emptyset}=f_{\emptyset}$ e $\psi_{\{1\}}((g, m))={ }^{g} \varphi_{\{1\}}(m)$. 
Temos que

$$
\begin{gathered}
\psi_{\{1\}}\left({ }^{h}(g, m)\right)=\psi_{\{1\}}((h g, m))={ }^{h g} \varphi_{\{1\}}(m)={ }^{h} \psi_{\{1\}}((g, m)) \\
\psi_{\{1\}} \circ f_{\{1\}}(m)=\psi_{\{1\}}((1, m))=\varphi_{\{1\}}(m)
\end{gathered}
$$

Agora seja $\sigma: f_{*} M \rightarrow N$ morfismo de módulo cruzado tal que $\sigma_{\emptyset}=f_{\emptyset}$ e $\sigma_{\{1\}} \circ f_{\{1\}}(m)=\varphi_{\{1\}}(m)$, então

$$
\sigma\{1\}((g, m))=\sigma\{1\}\left({ }^{g}(1, m)\right)={ }^{g} \sigma\{1\}((1, m))={ }^{g} \sigma \circ f_{\{1\}}(m)={ }^{g} \varphi_{\{1\}}(m)=\psi_{\{1\}}((g, m))
$$

Temos assim que $f_{*} M$ é um módulo cruzado induzido de $M$ por $f$.

Pelo teorema anterior temos como corolário que o módulo cruzado induzido em certo sentido generaliza a construção de um fecho normal de um subgrupo.

Corolario 1.5.6. Seja $G$ um grupo e $H \leq G$ um subgrupo de $G$. Considerando que o homomorfismo identidade $\mathbb{1}: H \rightarrow H$ junto com a a ação por conjugação forma um módulo cruzado, então a imagem do módulo cruzado induzido desse módulo cruzado pela inclusão $i: H \hookrightarrow G$ é isomorfo ao fecho normal de $H$ em $G$.

Demonstração: O fecho normal $\left\langle{ }^{G} H\right\rangle$ é gerado pelos elementos da forma ${ }^{g} h$ para $g \in G$ e $h \in H$. Defina o homomorfismo

$$
\begin{gathered}
\eta:\left\langle{ }^{G} H\right\rangle \rightarrow i_{*} H \\
{ }^{g} h \mapsto(g, h)
\end{gathered}
$$

É fácil ver pela apresentação dada pelo teorema 1.5.5 que $\eta$ e $\mu$ formam um isomorfismo de grupos.

Uma classe importante de exemplos de módulos cruzados induzidos são os módulos cruzados livres, dados pela definição abaixo, que como veremos no capítulo 4 são úteis para descrever o segundo grupo de homotopia relativo de um espaço obtido colando 2-células em um subespaço.

Definição 1.5.7. Dado um grupo $G$, um conjunto $R$ e uma função $w: R \rightarrow G$ o módulo cruzado livre gerado pela função $w: R \rightarrow G$, denotado $\mu: F(w) \rightarrow G$, é o módulo cruzado induzido do módulo cruzado cujos grupos são ambos o grupo livre $F(R)$ nos elementos de $R$, com o homomorfismo identidade e ação por conjugação, pelo homomorfismo $F(R) \rightarrow G$ induzido pela função $w$. O grupo $F(w)$ é gerado por $G \times R$ e tem relações dadas por

$$
\left(g^{\prime}, r^{\prime}\right)(g, r)\left(g^{\prime}, r^{\prime}\right)^{-1}=\left(g^{\prime} w\left(r^{\prime}\right) g^{\prime-1} g, r\right)
$$

a ação de $G$ em $F(w)$ é dada por ${ }^{h}(g, r)=(h g, r)$ e $\mu((g, r))=g r g^{-1}$.

Temos que um módulo cruzado induzido por uma sobrejeção tem uma apresentação simples dada por um quociente.

Teorema 1.5.8. Se $M$ é um módulo cruzado e $f_{\emptyset}: M_{\emptyset} \rightarrow G$ é um homomorfismo sobrejetor, então $f_{\emptyset *} M_{\{1\}} \cong \frac{M_{\{1\}}}{\left[K, M_{\{1\}}\right]}$, aonde $K=\operatorname{Ker} f_{\emptyset} e\left[K, M_{\{1\}}\right]$ denota o subgrupo de $M_{\{1\}}$ gerado por ${ }^{k} a a^{-1}$ para todo $a \in M_{\{1\}}$ e $k \in K$. 
Demonstração: Note que para quaisquer $n, n^{\prime} \in M_{\emptyset}$ tais que $f_{\emptyset}(n)=f_{\emptyset}\left(n^{\prime}\right)$ temos que $n^{-1} n^{\prime} \in$ $K$ e portanto temos que em $\frac{M_{\{1\}}}{\left[M_{\{1\}}, K\right]}$

$$
\begin{gathered}
{ }^{n^{-1} n^{\prime}} \bar{m} \bar{m}^{-1}=1 \\
n^{-1} n^{\prime} \bar{m}=\bar{m} \\
n^{\prime} \bar{m}={ }^{n} \bar{m}
\end{gathered}
$$

logo temos uma ação de $G$ sobre $\frac{M_{\{1\}}}{\left[K, M_{\{1\}}\right]}$ bem definida dada por ${ }^{g} \bar{m}={ }^{n} \bar{m}$ para algum $n \in f_{\emptyset}^{-1}(g)$.

Além disso note que $f_{\emptyset} \circ \mu$ induz um homomorfismo $\overline{f_{\emptyset} \circ \mu}: \frac{M_{\{1\}}}{\left[K, M_{\{1\}}\right]} \rightarrow G$ tal que

$$
\begin{gathered}
\overline{f_{\emptyset} \circ \mu}\left({ }^{g} \bar{m}\right)=\overline{f_{\emptyset} \circ \mu}\left({ }^{n} \bar{m}\right)=f_{\emptyset}(n) \overline{f_{\emptyset} \circ \mu}(\bar{m}) f_{\emptyset}(n)^{-1}=g \overline{f_{\emptyset} \circ \mu}(\bar{m}) g^{-1} \\
\bar{m}^{\prime} \bar{m} \bar{m}^{\prime-1}={ }^{\mu\left(m^{\prime}\right)} \bar{m}=\overline{f_{\emptyset} \circ \mu}\left(\bar{m}^{\prime}\right) \bar{m}
\end{gathered}
$$

portanto temos de fato uma estrutura de módulo cruzado.

Esse módulo cruzado satisfaz a propriedade universal dos módulos cruzados induzidos já que, para $\varphi: M \rightarrow N$ um morfismo de módulos cruzados com $N_{\emptyset}=G$ tal que $\varphi_{\emptyset}=f_{\emptyset}$, podemos definir $\psi_{\{1\}}: \frac{M_{\{1\}}}{\left[K, M_{\{1\}}\right]} \rightarrow N_{\{1\}}$ por $\psi_{\{1\}}(\bar{m})=\varphi_{\{1\}}(m)$ tal que

$$
\begin{aligned}
& \psi_{\{1\}}\left({ }^{g} \bar{m}\right)=\varphi_{\{1\}}\left({ }^{f_{\emptyset}(n)} m\right)={ }^{g} \varphi_{\{1\}}(m) \\
& \psi_{\{1\}} \circ f_{\{1\}}(m)=\psi_{\{1\}}(\bar{m})=\varphi_{\{1\}}(m)
\end{aligned}
$$

aonde $f_{\{1\}}$ é o homomorfismo quociente. Agora seja $\sigma_{\{1\}}: \frac{M_{\{1\}}}{\left[K, M_{\{1\}}\right]} \rightarrow N_{\{1\}}$ compatível com a ação de $G$ e com $\sigma_{\{1\}} \circ f_{\{1\}}(m)=\varphi_{\{1\}}(m)$, então claramente

$$
\sigma_{\{1\}}(\bar{m})=\sigma_{\{1\}} \circ f_{\{1\}}(m)=\varphi_{\{1\}}(m)=\psi_{\{1\}}(\bar{m})
$$

Temos assim que $\frac{M_{\{1\}}}{\left[K, M_{\{1\}}\right]} \rightarrow G$ é um módulo cruzado induzido de $M$ por $f_{\emptyset}$.

Também temos uma apresentação alternativa para o caso dos módulos cruzados induzidos por injeções. Para esse resultado usaremos as seguintes definições e notações. Seja $G$ um grupo e $T$ um conjunto. Definimos a copotência $T \vec{*} G$ como o produto livre de grupos $G_{t}, t \in T$, cada um com elementos $(t, g), g \in G$, e isomorfos a $G$ pela aplicação $(t, g) \rightarrow g$. Se $H$ é um grupo, então $H \vec{*} G$ denotará a copotência de $G$ pelo conjunto subjacente ao grupo $H$.

Teorema 1.5.9. Se $M$ é um módulo cruzado e $f_{\emptyset}: M_{\emptyset} \rightarrow G$ é um monomorfismo, seja $T$ uma transversal à esquerda de $f_{\emptyset}\left(M_{\emptyset}\right)$ em $G$. Tome a ação de $G$ sobre $T \vec{*} M_{\{1\}}$ dada por ${ }^{g}(t, m)=$ $\left(u,{ }^{n} m\right)$, aonde $n \in M_{\emptyset}, u \in T$ e gt $=u f_{\emptyset}(n)$. Seja $\delta: T \vec{*} M_{\{1\}} \rightarrow G$ definido por

$$
(t, m) \mapsto t f_{\emptyset} \circ \mu(m) t^{-1}
$$

Seja $S$ um conjunto de geradores do grupo $M_{\{1\}}$, e seja ${ }^{M_{\emptyset}} S=\left\{{ }^{n} x \mid x \in S, n \in M_{\emptyset}\right\}$. Então

$$
f_{\emptyset *} M_{\{1\}} \cong \frac{T \vec{*} M_{\{1\}}}{R}
$$


aonde $R$ é o fecho normal em $T \vec{*} M_{\{1\}}$ dos comutadores de Peiffer

$$
\left\langle(t, r),\left(t^{\prime}, r^{\prime}\right)\right\rangle=(t, r)\left(t^{\prime}, r^{\prime}\right)(t, r)^{-1} \delta((t, r))\left(t^{\prime}, r^{\prime}\right)^{-1}, r, r^{\prime} \in{ }^{M_{\emptyset}} S, t, t^{\prime} \in T .
$$

Demonstração: A partir da apresentação do teorema 1.5.5 e da hipótese que $T$ é transversal à esquerda de $f_{\emptyset}\left(M_{\emptyset}\right)$ temos que para todo par $(g, m) \in G \times M_{\{1\}}$ existe um $t \in T$ e um $n \in M_{\emptyset}$ tais que $(g, m)=\left(t f_{\emptyset}(n), m\right)=\left(t,{ }^{n} m\right)$. Portanto temos que $f_{\emptyset *} M_{\{1\}}$ é o quociente de $T \vec{*} M_{\{1\}}$ pelos comutadores de Peiffer $\left\langle T \vec{*} M_{\{1\}}, T \vec{*} M_{\{1\}}\right\rangle$. Como $T \vec{*} M_{\{1\}}$ é gerado pelo conjunto $T \times{ }^{M_{\emptyset}} S$ e esse conjunto é $G$-invariante já que ${ }^{g}\left(t,{ }^{n} m\right)=\left(u,{ }^{n} n m\right)$, aonde $g t=u f_{\emptyset}\left(n^{\prime}\right)$. Segue então que o fecho normal $R$ coincide com $\left\langle T \vec{*} M_{\{1\}}, T \vec{*} M_{\{1\}}\right\rangle$ [BHS11, prop. 3.3.5].

Pelos dois teoremas anteriores e o fato que todo homomorfismo de grupos pode ser decomposto em uma sobrejeção seguida de uma injeção obtemos um método para calcular módulos cruzados induzidos.

Em [BW95] encontramos um resultado que estabelece o módulo cruzado induzido sob algumas condições abelianas. Antes, mais algumas definições e notações. Seja $G$ um grupo e $T$ um conjunto. Definimos a copotência direta $T \vec{\oplus} G$ como a soma direta de grupos $G_{t}, t \in T$, cada um com elementos $(t, g), g \in G$, e isomorfos a $G$ pela aplicação $(t, g) \rightarrow g$.

Corolario 1.5.10. Seja $M$ um módulo cruzado e $f_{\emptyset}: M_{\emptyset} \rightarrow G$ um monomorfismo de grupos tais que $M_{\{1\}}$ é abeliano e $f_{\emptyset} \circ \mu\left(M_{\{1\}}\right)$ é normal em $G$, e seja $T$ uma transversal à esquerda de $f_{\emptyset}\left(M_{\emptyset}\right)$. Então $f_{\emptyset *} M_{\{1\}}$ é abeliano e é a copotência direta $T \vec{\oplus} G$.

Demonstração: Se $t, t^{\prime} \in T$ e $x \in{ }^{M_{\emptyset}} S$, então $\delta((t, r)) u=t f_{\emptyset} \circ \mu(r) t^{-1} u=t t^{-1} u f_{\emptyset} \circ \mu(m)=$ $u f_{\emptyset} \circ \mu(m)$ para algum $m \in M_{\{1\}}$ pela hipótese de normalidade. Portanto ${ }^{\delta(t, r))}\left(t^{\prime}, r^{\prime}\right)=\left(t^{\prime},{ }^{\mu(m)} r^{\prime}\right)=$ $\left(t^{\prime}, m r^{\prime} m^{-1}\right)=\left(t^{\prime}, r^{\prime}\right)$, logo o comutador de Peiffer reduz para o comutador usual

\subsection{Produtos tensoriais não-abelianos}

Nessa seção iremos definir o produto tensorial $G \otimes H$ para grupos (não necessariamente abelianos) $G$ e $H$ que agem um no outro a esquerda, e estabelecer sua relação com 2-cubos cruzados de grupos. Essa estrutura na forma apresentada aqui foi introduzidos pela primeira vez em [BL87a]. No presente trabalho usaremos apenas resultados básicos que podem ser encontrados no artigo mencionado, para mais resultados envolvendo esse produto tensorial vide a revisão da literatura em [Ka99] e uma bibliografia extensa no site do Brown [Bro] que, até o momento que o presente trabalho foi escrito, ele mantém atualizado.

Sejam $G$ e $H$ grupos tais que cada um age no outro pela esquerda. Sempre assumimos que um grupo age sobre si mesmo a esquerda por conjugação. Dadas tais ações o produto livre $G * H$ age em ambos $G$ e $H$, de forma que

$$
\begin{aligned}
& \left.{ }^{(h} g\right) g^{\prime}={ }^{h} g g^{\prime h} g^{-1}={ }^{h}\left(g^{h^{-1}} g^{\prime} g^{-1}\right)={ }^{h g h^{-1}} g^{\prime} ; \\
& \left.{ }^{(g} h\right) h^{\prime}={ }^{g} h h^{\prime g} h^{-1}={ }^{g}\left(h^{g^{-1}} h^{\prime} h^{-1}\right)=g h g^{-1} h^{\prime} .
\end{aligned}
$$

Diremos que as ações de $G$ e $H$ são compatíveis se

$$
\begin{aligned}
& { }^{(g h)} g^{\prime}={ }^{g h g^{-1}} g^{\prime} ; \\
& { }^{(h g)} h^{\prime}=h g h^{-1} h^{\prime} .
\end{aligned}
$$


Definição 1.6.1. O produto tensorial $G \otimes H$ é o grupo gerado pelos elementos de $G \times H$, que denotaremos por símbolos $(g \otimes h)$ para $g \in G$ e $h \in H$, com relações

$$
\begin{aligned}
& \left(g g^{\prime} \otimes h\right)=\left({ }^{g} g^{\prime} \otimes{ }^{g} h\right)(g \otimes h) \\
& \left(g \otimes h h^{\prime}\right)=(g \otimes h)\left({ }^{h} g \otimes{ }^{h} h^{\prime}\right) .
\end{aligned}
$$

Da mesma forma que o produto tensorial usual entre grupos abelianos transforma homomorfismos bilineares em homomorfismos lineares no produto tensorial, existe uma classe de funções cujo domínio é o produto de dois grupos que agem entre si pela esquerda que são transformados em homomorfismos no produto tensorial não-abeliano. Essa classe de funções é dada na definição a seguir.

Definição 1.6.2. Sejam $G$ e $H$ como antes e $L$ um grupo. Uma biderivação de $G \times H$ em $L$ é uma função $b: G \times H \rightarrow L$ tal que

$$
\begin{gathered}
b\left(g g^{\prime}, h\right)=b\left({ }^{g} g^{\prime},{ }^{g} h\right) b(g, h) \\
b\left(g, h h^{\prime}\right)=b(g, h) b\left({ }^{h} g,{ }^{h} h^{\prime}\right) .
\end{gathered}
$$

Claramente a função $G \times H \rightarrow G \otimes H$ com $(g, h) \mapsto(g \otimes h)$ é a biderivação universal no sentido que para qualquer biderivação $b: G \times H \rightarrow L$ existe um único homomorfismo $b_{*}: G \otimes H \rightarrow L$ tal que $b_{*}((g \otimes h))=b(g, h)$.

Note as equações que definem o produto tensorial e as biderivações são semelhantes a relações que valem para comutadores em grupos. Dado que os axiomas de $n$-cubos cruzados de grupos também se assemelham a relações de comutadores isso sugere uma relação entre essas estruturas que iremos explorar. Mostraremos agora como o produto tensorial não-abeliano possui duas estruturas de módulos cruzados, e veremos como elas se relacionam para formar um 2-cubo cruzado de grupos.

Teorema 1.6.3. Sejam $G$ e $H$ grupos com ações compativeis um no outro.

i) O produto livre $G * H$ age sobre $G \otimes H$ de forma que

$$
{ }^{x}(g \otimes h)=\left({ }^{x} g \otimes{ }^{x} h\right)
$$

ii) Existem homomorfismos

$$
\lambda: G \otimes H \rightarrow G, \lambda^{\prime}: G \otimes H \rightarrow H
$$

tais que $\lambda((g \otimes h))=g^{h} g^{-1}$ e $\lambda^{\prime}((g \otimes h))={ }^{g} h h^{-1}$

iii) Os homomorfismos $\lambda$ e $\lambda^{\prime}$, com as ações dadas, são módulos cruzados.

iv) $S e l \in G \otimes H$, então

$$
\begin{gathered}
(\lambda(l) \otimes h)=l^{h} l^{-1} \\
\left(g \otimes \lambda^{\prime}(h)\right)={ }^{g} l l^{-1}
\end{gathered}
$$

v) As ações de $G$ em $\operatorname{Ker} \lambda^{\prime}$ e de $H$ em Ker $\lambda$ são triviais. 
vi) se $l, l^{\prime} \in G \otimes H$, então

$$
\left[l, l^{\prime}\right]=\left(\lambda(l) \otimes \lambda^{\prime}\left(l^{\prime}\right)\right)
$$

em particular $\left[(g \otimes h),\left(g^{\prime} \otimes h^{\prime}\right)\right]=\left(g^{h} g^{-1} \otimes g^{\prime} h^{\prime} h^{\prime-1}\right)$.

Demonstração: i) Temos de fato uma ação já que

$$
\begin{aligned}
{ }^{x}\left(g g^{\prime} \otimes h\right) & =\left({ }^{x}\left(g g^{\prime}\right) \otimes{ }^{x} h\right) \\
& =\left({ }^{x} g^{x} g^{\prime x} g^{-1} \otimes{ }^{x} g\left({ }^{x} h\right)\right)\left({ }^{x} g \otimes{ }^{x} h\right) \\
& =\left({ }^{x}\left(g g^{\prime} g^{-1}\right) \otimes{ }^{x}\left({ }^{g} h\right)\right)\left({ }^{x} g \otimes{ }^{x} h\right) \\
& ={ }^{x}\left(g g^{\prime} g^{-1} \otimes{ }^{g} h\right)^{x}(g \otimes h)
\end{aligned}
$$

Note que a compatibilidade das ações é indispensável na passagem da segunda para a terceira linha. A outra igualdade relevante é análoga.

ii) Note que a função

$$
\begin{aligned}
\lambda: M \times N & \rightarrow M \\
(m, n) & \mapsto m^{n} m^{-1}
\end{aligned}
$$

é uma biderivação já que

$$
\begin{aligned}
\lambda\left(m m^{\prime}, n\right) & =m m^{\prime n}\left(m m^{\prime}\right)^{-1} \\
& =m m^{\prime} m^{-1} m^{n}\left(m^{\prime-1}\right) m^{-1} m^{n}\left(m^{-1}\right) \\
& =m m^{\prime} m^{-1} m n m^{-1}\left(m m^{-1} m^{-1}\right) m^{n}\left(m^{-1}\right) \\
& =\lambda\left(m n m^{-1},{ }^{m} n\right) \lambda(m, n)
\end{aligned}
$$

Logo pela propriedade universal $\lambda$ define um homomorfismo $\lambda: M \otimes N \rightarrow M$ tal que $\lambda(m \otimes n)=$ $m^{n} m^{-1}$. A demonstração para $\lambda^{\prime}$ é análoga.

iii) Vemos que $\lambda$ e a ação de $M$ em $M \otimes N$ satisfazem (MC1) já que

$$
\begin{aligned}
\lambda\left({m^{\prime}}^{\prime}(m \otimes n)\right) & =\lambda\left({ }^{m^{\prime}} m \otimes{ }^{m^{\prime}} n\right) \\
& =m^{\prime} m m^{-1} m^{\prime} n m^{\prime-1}\left(m^{\prime} m m^{-1}\right) \\
& =m^{\prime} m m^{-1} m^{\prime} n m \\
& =m^{\prime} m m^{-1} m^{\prime n} m m^{-1} \\
& =m^{\prime} m^{n} m m^{-1} \\
& =m^{\prime} \lambda(m \otimes n) m^{\prime-1}
\end{aligned}
$$

Para ver que $\lambda$ e a ação de $M$ em $M \otimes N$ satisfazem (MC2) note que temos duas formas de expandir $\left(m m^{\prime} \otimes n n^{\prime}\right)$, e que essas duas formas nos dão a equação

$$
{ }^{m n}\left(m^{\prime} \otimes n^{\prime}\right)(m \otimes n)=(m \otimes n)^{n m}\left(m^{\prime} \otimes n^{\prime}\right)
$$


e portanto temos

$$
\begin{aligned}
& \lambda(m \otimes n)\left(m^{\prime} \otimes n^{\prime}\right)={ }^{m^{n} m^{-1}}\left(m^{\prime} \otimes n^{\prime}\right) \\
& ={ }^{m n}\left(m^{-1} n^{-1} m^{\prime} \otimes m^{-1} n^{-1} n^{\prime}\right) \\
& =(m \otimes n)^{n m}\left(m^{-1} n^{-1} m^{\prime} \otimes m^{-1} n^{-1} n^{\prime}\right)(m \otimes n)^{-1} \\
& =(m \otimes n)\left(m^{\prime} \otimes n^{\prime}\right)(m \otimes n)^{-1}
\end{aligned}
$$

A demonstração para $\lambda^{\prime}$ e a ação de $N$ em $M \otimes N$ segue analogamente.

iv) Provaremos esse item por indução no comprimento da palavra $l$. Se $l=(g \otimes h)$ temos que

$$
\begin{aligned}
\left(\lambda(g \otimes h) \otimes h^{\prime}\right) & =\left(g^{h} g^{-1} \otimes h^{\prime}\right) \\
& ={ }^{g}\left({ }^{h} g^{-1} \otimes h^{\prime}\right)\left(g \otimes h^{\prime}\right) \\
& ={ }^{g h}\left(g^{-1} \otimes h^{-1} h^{\prime} h\right)\left(g \otimes h^{\prime}\right) \\
& ={ }^{g h}\left(g^{-1} \otimes h^{-1}\right)^{g}\left(g^{-1} \otimes h^{\prime} h\right)\left(g \otimes h^{\prime}\right) \\
& ={ }^{g}\left(g^{-1} \otimes h\right)^{-1}{ }^{g}\left(g^{-1} \otimes h^{\prime}\right)^{g h^{\prime}}\left(g^{-1} \otimes h\right)\left(g \otimes h^{\prime}\right) \\
& =(g \otimes h)\left(g \otimes h^{\prime}\right)^{-1 g h^{\prime}}\left(g^{-1} \otimes h\right)\left(g \otimes h^{\prime}\right) \\
& =(g \otimes h)^{h^{\prime} g}\left(g^{-1} \otimes h\right) \\
& =(g \otimes h)^{h^{\prime}}(g \otimes h)^{-1}
\end{aligned}
$$

Agora assuma que o item iv) vale para $l$ e $l^{\prime}$. Temos que

$$
\begin{aligned}
\left(\lambda\left(l l^{\prime}\right) \otimes h^{\prime}\right) & ={ }^{\lambda(l)}\left(\lambda\left(l^{\prime}\right) \otimes h^{\prime}\right)(\lambda(l) \otimes h) \\
& ={ }^{\lambda(l)}\left(l^{h^{\prime}} l^{\prime-1}\right)\left(l^{h^{\prime}} l^{-1}\right) \\
& \left.=l l^{\prime h^{\prime}} l^{\prime-1} l^{-1} l^{h^{\prime}} l^{-1}\right) \\
& =l l^{\prime h^{\prime}}\left(l l^{\prime}\right)^{-1}
\end{aligned}
$$

v) Seja $l \in \operatorname{Ker} \lambda$ e $h \in H$. Temos então pelo item iv) que

$$
l^{h} l^{-1}=(\lambda(l) \otimes h)=\left(1_{G} \otimes h\right)=1
$$

do que segue que ${ }^{h} l=l$.

vi) Para $l, l^{\prime} \in G \otimes H$ temos pelos itens iii) e $\left.i v\right)$ que

$$
\left[l, l^{\prime}\right]=l l^{\prime} l^{-1} l^{-1}=l^{\lambda^{\prime}\left(l^{\prime}\right)} l^{-1}=\left(\lambda(l) \otimes \lambda^{\prime}\left(l^{\prime}\right)\right)
$$

Como consequência do resultado anterior veremos como o produto tensorial não-abeliano pode ser usado para criar um 2-cubo cruzado de grupos a partir de dois módulos cruzados com o mesmo grupo indexado por $\emptyset$, e como esse cubo cruzado é obtido de um pushout. Assim como no caso do módulo cruzado induzido, isso sugere a utilidade dessa construção para aplicações do teorema de Seifert-van Kampen generalizado.

Teorema 1.6.4. Sejam $\mu: G \rightarrow K$ e $\nu: H \rightarrow K$ módulos cruzados, de forma que $G$ e $H$ ambos 
agem sobre $G$ e $H$ via $K$. Então existe um 2-cubo cruzado de grupos

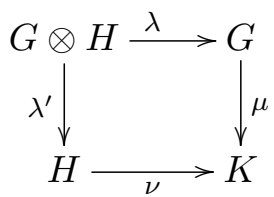

aonde $\lambda((g \otimes h))=g^{h} g^{-1}, \lambda^{\prime}((g \otimes h))={ }^{g} h h^{-1}$ e $h(g, h)=(g \otimes h)$. Esse módulo cruzado é universal no sentido de satisfazer as seguintes condições equivalentes:

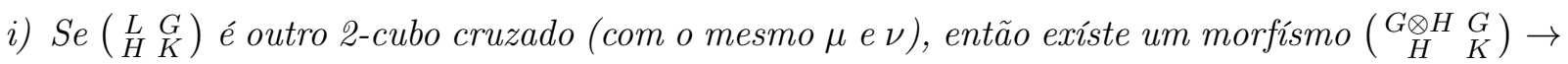
$\left(\begin{array}{cc}L & G \\ H & K\end{array}\right)$ de 2-cubos cruzados que é a identidade em $G, H$ e $K$.

ii) O seguinte diagrama de inclusões de 2-cubos cruzados é um pushout:

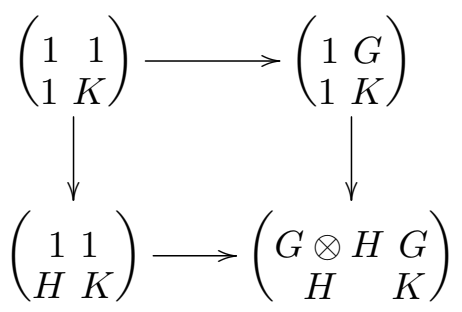

Demonstração: Que esse quadrado de grupos forma um 2-cubo cruzado de grupos segue imediatamente do teorema 1.6.3. Usando o corolário 1.4.3 para estabelecer que o pushout em ii) deve ser da forma $\left(\begin{array}{cc}? & G \\ H & K\end{array}\right)$ podemos usar as propriedades universais do pushout e do produto tensorial não abeliano para obter um isomorfismo que nos dá a equivalência de i) e ii). Que i) é satisfeito segue do fato que o morfismo de 2-cubos cruzados é totalmente definido pela biderivação $h: G \times H \rightarrow L$.

Pelo resultado a seguir, vemos que o produto tensorial não-abeliano generaliza o produto tensorial usual entre grupos abelianos.

Teorema 1.6.5. Se $G$ age trivialmente em $H$ e $H$ age trivialmente em $G$, então

$$
G \otimes H=G^{a b} \otimes_{\mathbb{Z}} H^{a b}
$$

aonde $\otimes_{\mathbb{Z}}$ é o produto tensorial usual de grupos abelianos.

Demonstração: Temos que nesse caso $\lambda$ e $\lambda^{\prime}$ são homomorfismos triviais, já que, por exemplo $\lambda(m \otimes n)=m^{n} m^{-1}=m m^{-1}=1$, logo pelo Teorema 1.6.3.v) temos que $M$ e $N$ agem trivialmente em $M \otimes N$ e portanto pelo segundo axioma de módulos cruzados segue que $M \otimes N$ é abeliano. Além disso as relações da definição do produto tensorial não abeliano se tornam

$$
\begin{aligned}
& \left(m m^{\prime} \otimes n\right)=(m \otimes n)+\left(m^{\prime} \otimes n\right) \\
& \left(m \otimes n n^{\prime}\right)=(m \otimes n)+\left(m \otimes n^{\prime}\right)
\end{aligned}
$$

e a definição de biderivação se torna a mesma do que a de transformações bilineares. 
Existe então transformações bilineares

$$
\begin{aligned}
M^{\mathrm{ab}} \times N^{\mathrm{ab}} & \rightarrow M \otimes N \\
(\bar{m}, \bar{n}) & \mapsto(m \otimes n) \\
M \times N & \rightarrow M^{\mathrm{ab}} \otimes_{\mathbb{Z}} N^{\mathrm{ab}} \\
(m, n) & \mapsto\left(\bar{m} \otimes_{\mathbb{Z}} \bar{n}\right)
\end{aligned}
$$

bem definidos já que

$$
\begin{aligned}
\left(m m^{\prime} m^{-1} m^{-1} \otimes n\right) & =(m \otimes n)+\left(m^{\prime} \otimes n\right)+\left(m^{-1} \otimes n\right)+\left(m^{-1} \otimes n\right) \\
& =(m \otimes n)+\left(m^{-1} \otimes n\right)+\left(m^{\prime} \otimes n\right)+\left(m^{-1} \otimes n\right) \\
& =1
\end{aligned}
$$

que induzem homomorfismos inversos nos produtos tensoriais

\section{$1.7 n$-Cubos cruzados $r$-universais}

Para algumas aplicações do teorema generalizado de Seifert-van Kampen que apresentaremos precisaremos da definição de um $n$-cubo cruzado $r$-universal, que pode ser pensado como o $n$-cubo cruzado gerado livremente pelos $M_{A} \operatorname{com}|A| \leq r$, e que foi introduzido pela primeira vez em [ES87]. Precisaremos primeiro de uma definição que será útil.

Definição 1.7.1. Um $(r, n)$-canto cruzado de grupos $M^{r}$ para $r \leq n$ é composto por uma família de grupos $M_{A}^{r}$, uma família de homomorfismos $\mu_{i}: M_{A}^{r} \rightarrow M_{A \backslash\{i\}}^{r}$ e uma família de funções $h: M_{A}^{r} \times M_{B}^{r} \rightarrow M_{A \cup B}^{r}$ para $A, B \subseteq\langle n\rangle$ com $|A| \leq r$ e $|A \cup B| \leq r$ que satisfaçam (I ${ }^{n} \mathrm{CG} 1$ )( ${ }^{n}$ CG11). Um morfismo de $(r, n)$-cantos cruzados de grupos $f^{r}: M^{r} \rightarrow N^{r}$ é uma família de homomorfismo $f_{A}^{r}: M_{A}^{r} \rightarrow N_{A}^{r}$ para $|A| \leq r$ que comutam com as funções $h$ e $\mu_{i}$.

Todo $(r, n)$-canto cruzado de grupos $M^{r}$ define um $n$-cubo cruzado de grupos $M$ aonde $M_{A}=$ $M_{A}^{r}$ se $|A| \leq r$ e $M_{A}=1$ se $|A|>r$. Além disso todo $n$-cubo cruzado de grupos $M$ define um $(r, n)$-canto cruzado de grupos $M^{r}$ simplesmente ignorando os grupos $M_{A}$ com índices tais que $|A|>r$.

Definição 1.7.2. Um $n$-cubo cruzado de grupos $M$ é $r$-universal se para todo $n$-cubo cruzado $N$ tal que $M^{r}=N^{r}$ existe um único homomorfismo $M \rightarrow N$ que é a identidade nos $M_{A}$ para $|A| \leq r$.

Veremos agora como os $n$-cubos cruzados $r$-universais surgem como colimites de certos diagramas de $n$-cubos cruzados de grupos.

Teorema 1.7.3. Tome $M^{r}$ um $(r, n)$-canto cruzado de grupos. Então para cada $\hat{A} \subseteq\langle n\rangle$ com $|\hat{A}| \leq r$ temos um $n$-cubo cruzado ${ }^{\hat{A}} M^{r}$ com

$$
\hat{A}_{M_{A}^{r}}=\left\{\begin{array}{ll}
1 & \text { se } A \cap \hat{A} \neq \emptyset \\
M_{A}^{r} & \text { se } A \cap \hat{A}=\emptyset
\end{array},\right.
$$

acrescentando funções triviais aonde forem necessárias. Também temos inclusões canônicas ${ }^{\hat{A}} M^{r} \hookrightarrow$ $\hat{A} \backslash\{i\} M^{r}$ para $|\hat{A}| \leq r-1$. Além disso temos que colim ${ }^{\hat{A}} M^{r}$ é um n-cubo cruzado r-universal. 


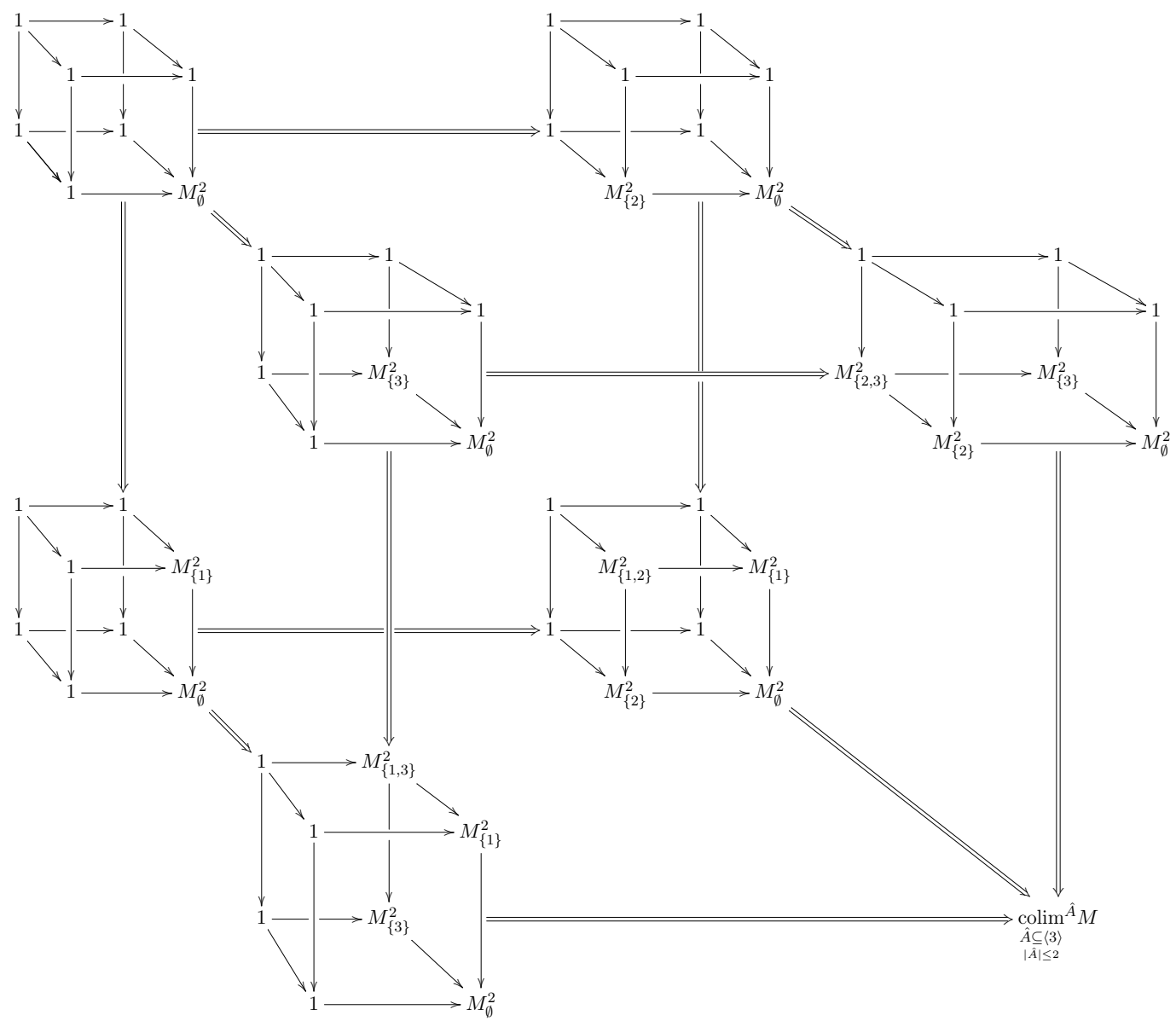

Figura 1.3: Diagrama de 3-cubos cruzados de grupos cujo colimite é um 3-cubo cruzado 2-universal.

Demonstração: A primeira parte do teorema é trivial dada a hipótese de que as funções satisfazem ( $\left.{ }^{n} \mathrm{CG} 1\right)-\left(\mathrm{I}^{n} \mathrm{CG} 11\right)$. Pela definição de $n$-cubo cruzado $r$-universal, para demonstrar a segunda parte basta provarmos que

$$
\left(\begin{array}{l}
\operatorname{colim}_{\hat{A} \subseteq\langle n\rangle} \hat{A} \\
|\hat{A}| \leq r
\end{array} M^{r}\right)_{A}=M_{A}^{r} \text { para todo } A \subseteq\langle n\rangle \operatorname{com}|A| \leq r .
$$

Pela definição dos funtores $\check{\partial}_{i}$ e pelo corolário $\mathbf{1 . 4 . 3}$ obtemos

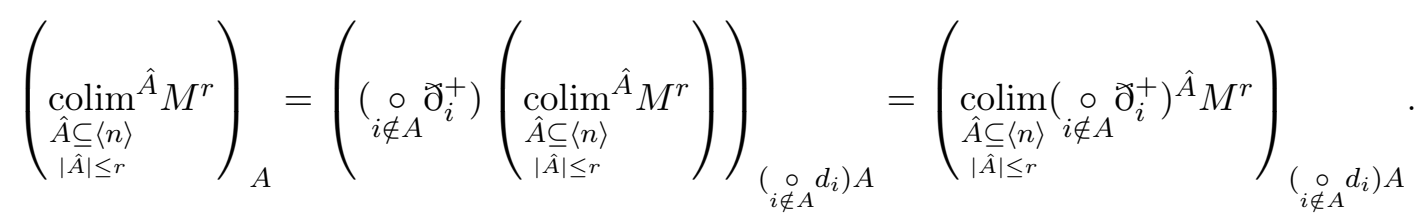

Como $\check{J}_{i}^{+}$manda as inclusões ${ }^{\hat{A}} M \hookrightarrow^{\hat{A} \backslash\{i\}} M$ para identidades temos

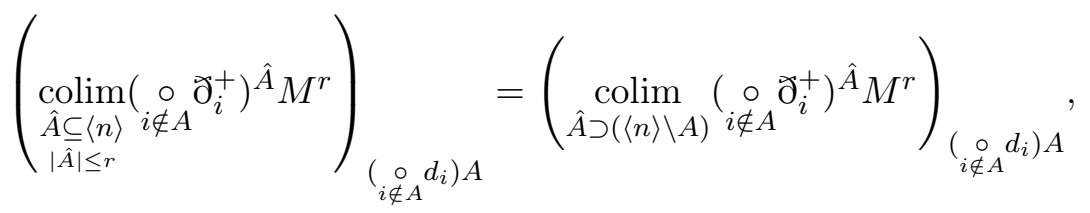

e como $\left.\underset{i \notin A}{\circ} \mathrm{J}_{i}^{+}\right)^{A} M^{r}$ é objeto terminal do diagrama $\left.\underset{i \notin A}{\circ} \mathrm{J}_{i}^{+}\right)^{\hat{A}} M^{r},(\hat{A} \supset(\langle n\rangle \backslash A))$ temos 


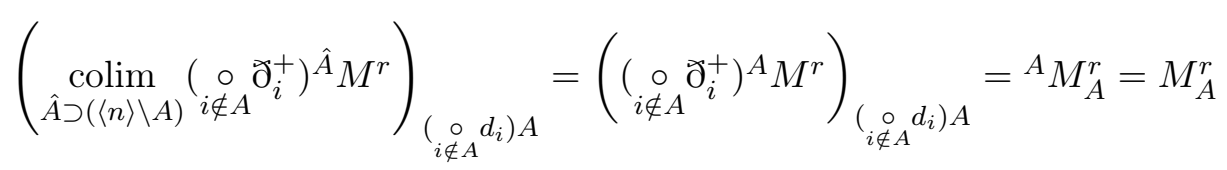

o que completa a demonstração

Existe uma apresentação na forma de quocientes de produtos livres de produtos tensoriais para os $n$-cubos cruzados $r$-universais dado pelo teorema abaixo, o que garante a existência desses colimites. Usaremos a notação $\mu a=\left(\underset{i \in A}{\circ} \mu_{i}\right) a \in M_{\emptyset}$. A ideia aqui é construir recursivamente os grupos $M_{A}$ para $|A|>r$ como produtos tensoriais de grupos indexados por subconjuntos $B, C \subset A$ disjuntos tais que $A=B \cup C$, de forma que os elementos desse produto tensorial sejam definidos como a imagem das funções $h$ nos elementos de $M_{B} \times M_{C}$. Precisaremos incluir algumas relações para garantir que a estrutura construída satisfaça os axiomas de $n$-cubos cruzados de grupos.

Teorema 1.7.4. Seja $M$ um n-cubo cruzado r-universal. Então podemos recursivamente dar uma apresentação dos $M_{A}$ para $|A|>r$ como sendo o quociente do produto livre dos produtos tensoriais $M_{B} \otimes M_{C}$ para $B \cup C=A, B \cap C=\emptyset, B, C \neq \emptyset$ pelas relações:

$$
\begin{aligned}
& b \otimes c=(c \otimes b)^{-1} \\
& \mu_{i} b \otimes c=b \otimes \mu_{i} c \\
& (b \otimes c)(d \otimes e)(b \otimes c)^{-1}=\left({ }^{[\mu b, \mu c]} d \otimes{ }^{[\mu b, \mu c]} e\right) \\
& \left({ }^{b} h\left(b^{-1}, c\right) \otimes{ }^{\mu b} d\right)\left({ }^{d} h\left(d^{-1}, b\right) \otimes{ }^{\mu d} c\right)\left({ }^{c} h\left(c^{-1}, d\right) \otimes{ }^{\mu c} b\right)=1
\end{aligned}
$$

Temos a ação de $M_{\emptyset}$ nos $M_{A}$ dadas por ${ }^{x}(b \otimes c)=\left({ }^{x} b \otimes{ }^{x} c\right)$. A função $h$ para $B$ e $C$ tais que $B \cup C>r$ é dada por

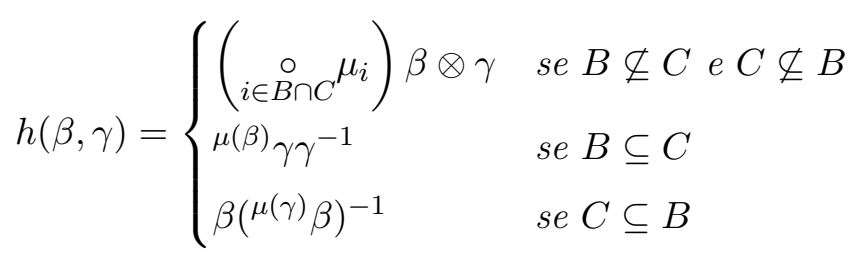

e $\mu_{i}((b \otimes c))=h\left(\mu_{i}(b), \mu_{i}(c)\right)$.

Demonstração: Podemos ver que essa construção descreve um $n$-cubo cruzado de grupos pois ( $\left.{ }^{n} \mathrm{CG} 1\right)-\left(\mathrm{I}^{n} \mathrm{CG} 4\right)$ e ( $\left.\mathrm{I}^{n} \mathrm{CG} 11\right)$ seguem por como os $\mu_{i}$ e $h$ são definidos junto com (rU2). O axioma ( $\left.{ }^{n} \mathrm{CG} 5\right)$ segue de (rU3), o ( $\left.{ }^{n} \mathrm{CG} 6\right)$ de (rU1) e ( $\left.{ }^{n} \mathrm{CG} 10\right)$ de (rU4). Da definição de produto tensorial obtemos ( $\left.{ }^{n} \mathrm{CG} 7\right)-\left(\mathrm{I}^{n} \mathrm{CG} 9\right)$.

Seja $N$ um $n$-cubo cruzado tal que $N_{A}=M_{A}$ para $|A| \leq r$, então podemos definir recursivamente um morfismo de $n$-cubos cruzados $\psi: M \rightarrow N$ tal que $\psi_{A}=\mathbb{1}_{A}$, para $|A| \leq r$, e no caso $|A|>r$ definimos $\psi_{A}$ em cada um dos produtos tensoriais como o morfismo induzido das biderivações

$$
\begin{gathered}
M_{B} \times M_{C} \rightarrow N_{A} \\
(b, c) \mapsto h\left(\psi_{B}(b), \psi_{C}(c)\right)
\end{gathered}
$$

Podemos ver que pela definição dos produtos tensoriais e das funções $h$ esses homomorfismos estão bem definidos, e que pela propriedade universal dos produtos tensoriais eles são os únicos homomorfismos que formam um morfismo de $n$-cubos cruzados com as propriedades requeridas. 


\section{$1.8 n$-Cubos cruzados de grupos $r$-induzidos}

Podemos generalizar o conceito de um módulo cruzado induzido por um homomorfismo para o conceito de um $n$-cubo cruzado de grupos induzido por um morfismo de $(r, n)$-cantos cruzados de grupos. As definições e teoremas dessa seção são adaptações dos resultados sobre cat $^{n}$-grupos induzidos encontrados em [BL87b].

Definição 1.8.1. Sejam $M$ um $n$-cubo cruzado de grupos, $N^{r}$ um $(r, n)$-canto cruzado de grupos e $f^{r}: M^{r} \rightarrow N^{r}$ um morfismo de $(r, n)$-cantos cruzados de grupos. O $n$-cubo cruzado de grupos $r$ induzido de $M$ por $f^{r}$ é denotado por $f_{*}^{r} M$ e é definido pela propriedade universal que $\left(f_{*}^{r} M\right)^{r}=N^{r}$ e existe $f: M \rightarrow f_{*}^{r} M$ que estende $f^{r}$ tal que para todo morfismo de $n$-cubos cruzados de grupos $\phi: M \rightarrow L \operatorname{com} L^{r}=N^{r}$ e $g^{r}=f^{r}$ existe um único morfismo $\psi: f_{*}^{r} M \rightarrow L$ tal que $\psi \circ f=\phi$.

Vejamos como os $n$-cubos cruzados $r$-induzidos surgem como colimites de certos diagramas.

Teorema 1.8.2. Sejam $M$ um n-cubo cruzado de grupos, $N^{r}$ um $(r, n)$-canto cruzado de grupos e $f^{r}: M^{r} \rightarrow N^{r}$ um morfismo de $(r, n)$-cantos cruzados de grupos. Assim como fizemos no teorema 1.7.3 temos para todo $\hat{A} \subseteq\langle n\rangle$ com $|\hat{A}| \leq r$ os $n$-cubos cruzados de grupos ${ }^{\hat{A}} M^{r} e^{\hat{A}} N^{r}$ que formam dois diagramas como no teorema mencionado. Além disso temos morfismos ${ }^{\hat{A}} f^{r}:{ }^{\hat{A}} M^{r} \rightarrow{ }^{\hat{A}} N^{r}$ induzidos por $f^{r}$ e inclusões ${ }^{\hat{A}} M^{r} \rightarrow M$ para todo $\hat{A} \subseteq\langle n\rangle$ com $|\hat{A}| \leq r$. Todos esses morfismos formam um diagrama comutativo cujo colimite é $f_{*}^{r} M$.

Demonstração: A demonstração que o $(r, n)$-canto cruzado associado ao colimite desse diagrama é $N^{r}$ é análoga a demonstração do teorema 1.7.3. O resto da propriedade universal segue das definições de colimite e de $n$-cubo cruzado $r$-induzido de $M$ por $f^{r}$

Vejamos agora uma apresentação que garante a existência dos $n$-cubos cruzados de grupos $r$-induzidos.

Teorema 1.8.3. Sejam $M$ um n-cubo cruzado de grupos, $N^{r}$ um $(r, n)$-canto cruzado de grupos e $f^{r}: M^{r} \rightarrow N^{r}$ um morfismo de $(r, n)$-cantos cruzados de grupos. Então podemos recursivamente dar uma apresentação dos $\left(f_{*}^{r} M\right)_{A}$ para $|A|>r$ como sendo o quociente do produto livre do módulo cruzado induzido $f_{\emptyset *}^{r} M_{A}$ e dos produtos tensoriais não abelianos $\left(f_{*}^{r} M\right)_{B} \otimes\left(f_{*}^{r} M\right)_{C}$ para $B \cup C=$ $A, B \cap C=\emptyset, B, C \neq \emptyset$ pelas relações:

$$
\begin{gathered}
(b \otimes c)=(c \otimes b)^{-1} \\
\left(\mu_{i}(b) \otimes c\right)=\left(b \otimes \mu_{i}(c)\right) \\
\left(n, h\left(m, m^{\prime}\right)\right)=\left({ }^{n} f_{B}^{r}(m) \otimes{ }^{n} f_{C}^{r}\left(m^{\prime}\right)\right) \\
(b \otimes c)(n, m)(b \otimes c)^{-1}=([\mu(b), \mu(c)] n, m) \\
\left(b^{\prime} \otimes c^{\prime}\right)(b \otimes c)\left(b^{\prime} \otimes c^{\prime}\right)^{-1}=\left({ }^{\left[\mu\left(b^{\prime}\right), \mu\left(c^{\prime}\right)\right]} b \otimes{ }^{\left[\mu\left(b^{\prime}\right), \mu\left(c^{\prime}\right)\right]} c\right) \\
(n, m)(b \otimes c)(n, m)^{-1}=\left({ }^{\left.n \mu(m) n^{-1} b \otimes n \mu(m) n^{-1} c\right)}\right. \\
\left({ }^{\mu(b)}\left(b^{-1} \otimes c\right) \otimes{ }^{\mu(b)} d\right)\left({ }^{\mu(d)}\left(d^{-1} \otimes b\right) \otimes{ }^{\mu(d)} c\right)\left({ }^{\mu(c)}\left(c^{-1} \otimes d\right) \otimes\right.
\end{gathered}
$$


Temos a ação de $N_{\emptyset}$ nesses grupos dadas por

$$
\begin{gathered}
n^{n^{\prime}}(n, m)=\left(n^{\prime} n, m\right) \\
{ }^{n}(b \otimes c)=\left({ }^{n} b \otimes{ }^{n} c\right)
\end{gathered}
$$

As funções $h$ são dadas por

$$
h(\beta, \gamma)= \begin{cases}\left(\begin{array}{cl}
\circ \\
i \in B \cap C
\end{array} \mu_{i}\right) \beta \otimes \gamma & \text { se } B \nsubseteq C \text { e } C \nsubseteq B \\
\mu(\beta) \gamma \gamma^{-1} & \text { se } B \subseteq C \\
\beta\left({ }^{\mu(\gamma)} \beta\right)^{-1} & \text { se } C \subseteq B\end{cases}
$$

e as funções $\mu_{i}$ são dadas por

$$
\begin{gathered}
\mu_{i}((n, m))= \begin{cases}\left(n, \mu_{i}(m)\right) & \text { se } A \neq\{i\} \\
n \mu_{i}(m) n^{-1} & \text { se } A=\{i\}\end{cases} \\
\mu_{i}((b \otimes c))=h\left(\mu_{i}(b), \mu_{i}(c)\right)
\end{gathered}
$$

Demonstração: Podemos ver que essa construção descreve um $n$-cubo cruzado de grupos pois ( $\left.{ }^{n} \mathrm{CG} 1\right)-\left(\mathrm{I}^{n} \mathrm{CG} 4\right)$ e (I $\left.{ }^{n} \mathrm{CG} 11\right)$ seguem por como os $\mu_{i}$ e $h$ são definidos e usando (CCGI2). O axioma ( ${ }^{n}$ CG5) segue dos (CCGI4)-(CCGI6), o (I ${ }^{n}$ CG6) de (CCGI1) e (I ${ }^{n}$ CG10) de (CCGI7). Da definição de produto tensorial obtemos ( $\left.{ }^{n} \mathrm{CG} 7\right)-\left(\mathrm{I}^{n} \mathrm{CG} 9\right)$.

Temos o morfismo $f: M \rightarrow f_{*}^{r} M$ dado por $f_{A}(m)=(1, m)$ para $|A|>r$, e por $f_{A}(m)=f_{A}^{r}(m)$ no caso $|A| \leq r$. Pelas definições de módulos cruzados induzidos e de produtos tensoriais, e por (CCGI3) estas funções estão bem definidas e elas são homomorfismos de $n$-cubos cruzados de grupos.

Para qualquer homomorfismo de $n$-cubos cruzados de grupos $\phi: M \rightarrow L$ temos o homomorfismo $f_{*}^{r} \phi: f_{*}^{r} M \rightarrow L$ definido recursivamente nos $f_{A *}^{r} M_{A}$ como os morfismos induzidos de $\phi_{\emptyset}$ e nos $(M \circledast N)_{B} \otimes(M \circledast N)_{C}$ como os morfismos induzidos das biderivações

$$
\begin{gathered}
\left(f_{*}^{r} M\right)_{B} \times\left(f_{*}^{r} M\right)_{C} \rightarrow L_{A} \\
(b, c) \mapsto h\left(\left(f_{*}^{r} \phi\right)_{B}(b),\left(f_{*}^{r} \phi\right)_{C}(c)\right)
\end{gathered}
$$

Podemos ver que pela definição dos produtos tensoriais, módulos induzidos e das funções $h$ esses homomorfismos estão bem definidos, e que pela propriedade universal dos produtos tensoriais e dos módulos induzidos eles são os únicos homomorfismos que formam um morfismo de $n$-cubos cruzados com as propriedades requeridas.

Temos um caso particular que será útil para demonstrarmos uma certa generalização do teorema de Hurewicz no capítulo 4.

Corolario 1.8.4. Seja $M$ um n-cubo cruzado de grupos, $N^{n-1}$ um $(n-1, n)$-canto cruzado de grupos cujos grupos são todos triviais e $f^{n-1}$ o morfismo de $(n-1, n)$-cantos cruzados de grupos trivial de $M^{n-1}$ em $N^{n-1}$. Então $\left(f_{*}^{r} M\right)_{\langle n\rangle}$ é o quociente de $M_{\langle n\rangle}$ pelos subgrupos normais $h\left(M_{B}, M_{C}\right)$ para todo $B, C \subseteq\langle n\rangle$ tais que $B \cup C=\langle n\rangle$.

Demonstração: Para começar temos pelo teorema 1.5.8 que $f_{\emptyset *}^{r} M_{\langle n\rangle}=\frac{M_{\langle n\rangle}}{\left[M_{\emptyset}, M_{\langle n\rangle}\right]}=\frac{M_{\langle n\rangle}}{h\left(M_{\emptyset}, M_{\langle n\rangle}\right)}$. 
Como $N^{n-1}$ é trivial todos os produtos tensoriais da apresentação do teorema são triviais, logo no teorema a única relação não-trivial é a (CCGI3), o que nos dá o resultado que queríamos.

Denotamos o grupo $f_{*}^{r} M$ descrito no corolário por Triv $M$.

\subsection{Coprodutos}

Sejam $M$ e $N$-cubos cruzados de grupos. Daremos uma descrição do seu coproduto, denotado $M \circledast N$ usando uma técnica análoga á das últimas duas seções. Criaremos recursivamente grupos como produtos livres de produtos tensoriais e módulos induzidos por funções, e acrescentaremos as relações necessárias para que a estrutura final seja um $n$-cubo cruzado de grupos. Não foi encontrado na literatura uma descrição anterior dessa operação.

Note que pelo corolário 1.4.3 podemos concluir que o grupo indexado por $\emptyset$ nesse coproduto deve ser $M_{\emptyset} * N_{\emptyset}$. Denotaremos por $i: M_{\emptyset} \rightarrow M_{\emptyset} * N_{\emptyset}$ e $j: N_{\emptyset} \rightarrow M_{\emptyset} * N_{\emptyset}$ as inclusões canônicas do produto livre.

Teorema 1.9.1. O coproduto $M \circledast N$ é definido recursivamente no tamanho dos subconjuntos $A \subseteq\langle n\rangle$ como o quociente do produto livre dos módulos induzidos $i_{*} M_{A}$ e $j_{*} N_{A}$, e dos produtos tensoriais $(M \circledast N)_{B} \otimes(M \circledast N)_{C}$ para $B \cup C=A, B \cap C=\emptyset, B, C \neq \emptyset$ pelas relações:

$$
\begin{gathered}
(b \otimes c)=(c \otimes b)^{-1} \\
\left(\mu_{i}(b) \otimes c\right)=\left(b \otimes \mu_{i}(c)\right) \\
(x, h(k, l))=((x, k) \otimes(x, l)) \\
\left(x^{\prime}, k^{\prime}\right)(x, k)\left(x^{\prime}, k^{\prime}\right)^{-1}=\left(x^{\prime} \mu\left(k^{\prime}\right) x^{\prime-1} x, k\right) \\
(b \otimes c)(x, k)(b \otimes c)^{-1}=([\mu(b), \mu(c)] x, k) \\
\left(b^{\prime} \otimes c^{\prime}\right)(b \otimes c)\left(b^{\prime} \otimes c^{\prime}\right)^{-1}=\left({ }^{\left[\mu\left(b^{\prime}\right), \mu\left(c^{\prime}\right)\right]} b \otimes\left[\mu\left(b^{\prime}\right), \mu\left(c^{\prime}\right)\right] c\right) \\
(x, k)(b \otimes c)(x, k)^{-1}=\left({ }^{x \mu(k) x^{-1}} b \otimes{ }^{x \mu(k) x^{-1}} c\right) \\
\left({ }^{\mu(b)}\left(b^{-1} \otimes c\right) \otimes{ }^{\mu(b)} d\right)\left({ }^{\mu(d)}\left(d^{-1} \otimes b\right) \otimes{ }^{\mu(d)} c\right)\left({ }^{\mu(c)}\left(c^{-1} \otimes d\right) \otimes\right.
\end{gathered}
$$

Temos a ação de $M_{\emptyset} * N_{\emptyset}$ nesses grupos dadas por

$$
\begin{gathered}
{ }^{y}(x, k)=(y x, k) \\
{ }^{x}(b \otimes c)=\left({ }^{x} b \otimes{ }^{x} c\right)
\end{gathered}
$$

As funções $h$ são dadas por

$$
h(\beta, \gamma)= \begin{cases}\left(\begin{array}{ll}
\stackrel{\circ}{i \in B \cap C} \\
\mu_{i}
\end{array}\right) \beta \otimes \gamma & \text { se } B \nsubseteq C \text { e } C \nsubseteq B \\
\mu(\beta) \gamma \gamma^{-1} & \text { se } B \subseteq C \\
\left.\beta{ }^{\mu(\gamma)} \beta\right)^{-1} & \text { se } C \subseteq B\end{cases}
$$


e as funções $\mu_{i}$ são dadas por

$$
\begin{gathered}
\mu_{i}((x, k))= \begin{cases}\left(x, \mu_{i}(k)\right) & \text { se } A \neq\{i\} \\
x \mu_{i}(k) x^{-1} & \text { se } A=\{i\}\end{cases} \\
\mu_{i}((b \otimes c))=h\left(\mu_{i}(b), \mu_{i}(c)\right)
\end{gathered}
$$

Demonstração: Podemos ver que essa construção descreve um $n$-cubo cruzado de grupos pois ( $\left.{ }^{n} \mathrm{CG} 1\right)-\left(\mathrm{I}^{n} \mathrm{CG} 4\right)$ e ( $\left.\mathrm{I}^{n} \mathrm{CG} 11\right)$ seguem por como os $\mu_{i}$ e $h$ são definidos e usando (Cop2). O axioma ( ${ }^{n}$ CG5) segue dos (Cop4)-(Cop7), o ( ${ }^{n}$ CG6) de (Cop1) e ( ${ }^{n}$ CG10) de (Cop8). Da definição de produto tensorial obtemos ( $\left.{ }^{n} \mathrm{CG} 7\right)-\left(\mathrm{I}^{n} \mathrm{CG} 9\right)$.

Temos inclusões naturais $i: M \rightarrow M \circledast N$ e $j: N \rightarrow M \circledast N$ dadas por $i_{A}(m)=(1, m)$ e $j_{A}(n)=(1, n)$ para $m \in M_{A}$ e $n \in N_{A}$, com $A \subseteq\langle n\rangle$ e $A \neq \emptyset$, e com as inclusões usuais no caso $A=\emptyset$. Pelas definições de módulos cruzados induzidos e de produtos tensoriais, e por (Cop3) estas funções estão bem definidas e elas são homomorfismos de $n$-cubos cruzados de grupos.

Para quaisquer homomorfismos de $n$-cubos cruzados de grupos $\phi: M \rightarrow L$ e $\psi: N \rightarrow L$ temos o homomorfismo $\phi \circledast \psi: M \circledast N \rightarrow L$ definido recursivamente nos $i_{*} M_{A}$ e $j_{*} N_{A}$ como os morfismos induzidos de $\phi_{\emptyset} * \psi_{\emptyset}$ e nos $(M \circledast N)_{B} \otimes(M \circledast N)_{C}$ como os morfismos induzidos das biderivações

$$
\begin{gathered}
(M \circledast N)_{B} \times(M \circledast N)_{C} \rightarrow L_{A} \\
(b, c) \mapsto h\left((\phi \circledast \psi)_{B}(b),(\phi \circledast \psi)_{C}(c)\right)
\end{gathered}
$$

Podemos ver que pela definição dos produtos tensoriais, módulos induzidos e das funções $h$ esses homomorfismos estão bem definidos, e que pela propriedade universal dos produtos tensoriais e dos módulos induzidos eles são os únicos homomorfismos que formam um morfismo de $n$-cubos cruzados com as propriedades requeridas. 


\section{Capítulo 2}

\section{$n$-Cubos de espaços}

No trabalho de Whitehead vemos que para associar um módulo cruzado a um espaço precisamos primeiro escolher uma certa fibração. Seja

$$
F \rightarrow E \rightarrow X
$$

uma fibração aonde os três espaços são conexos, o espaço base $X$ é um 2-tipo homotópico e o espaço total $E$ e a fibra $F$ são 1-tipos homotópicos. Isso implica que a única parte não trivial da sequência exata dessa fibração é

$$
\cdots \rightarrow 0 \rightarrow \pi_{2}(X) \rightarrow \pi_{1}(F) \rightarrow \pi_{1}(E) \rightarrow \pi_{1}(X) \rightarrow * \rightarrow \cdots
$$

Como mencionamos na introdução, o módulo cruzado dessa fibração é definido como o homomorfismo $\partial: \pi_{1}(F) \rightarrow \pi_{1}(E)$, junto com a ação de $\pi_{1}(E)$ em $\pi_{1}(F)$. Seguindo essas ideias, para mostrar como usar as estruturas algébricas do capítulo $\mathbf{1}$ como invariantes topológicas precisaremos primeiro introduzir generalizações de fibrações. O presente capítulo segue a apresentação das definições e teoremas do artigo de Steiner [St86], que tinha como objetivo corrigir alguns erros no artigo de Loday [Lod82].

Na primeira seção introduziremos $n$-cubos de fibrações e $n$-cubos fibrantes, que podem ser pensados como generalizações de fibrações, e mostraremos a equivalência entre as categorias dessas duas estruturas topológicas. Assim como no caso das estruturas do capítulo anterior apresentamos duas categorias equivalentes pois certos resultados são mais facilmente demonstrados na categoria dos $n$-cubos fibrantes, porém mais tarde iremos querer aplica-los na categoria dos $n$-cubos de fibrações. Damos também uma definição de conectividade para essas estruturas que será de suma importância para as hipóteses de muitos teoremas. Daremos a definição de uma equivalência entre $n$-cubos de fibrações assim como o conceito de equivalência de espaço base, que será importante para estabelecer a relação dessas estruturas com os $(n+1)$-tipos homotópicos.

Na segunda seção veremos como todo espaço está associado a um $n$-cubo de fibrações canônico. Introduziremos a subcategoria dos $n$-cubos de Eilenberg-MacLane e mostraremos que se criarmos uma localização dessa subcategoria invertendo as equivalências de espaço base obtemos uma categoria equivalente à categoria homotópica dos $(n+1)$-tipos homotópicos.

\section{$2.1 n$-Cubos fibrantes e de fibrações}

Nessa seção apresentamos as definições das categorias de $n$-cubos fibrantes e a de $n$-cubos de fibrações. Veremos uma definição de conectividade para essas estruturas, que será importante pois o teorema generalizado de Seifert-Van Kampen só se aplica a estruturas conexas. A vantagem de 
trabalhar com $n$-cubos fibrantes é que eles são mais fáceis de se construir indutivamente e de garantir conectividade. Por outro lado os $n$-cubos de fibrações tem a vantagem de ter uma definição mais transparente do que é seu $n$-cubo cruzado de grupos fundamental.

Vejamos primeiro como definiremos um $n$-cubo de espaços.

Definição 2.1.1. Um $n$-cubo de espaços $X$ é uma família de espaços $X_{B}$ indexados pelos subconjuntos $B \subseteq\langle n\rangle$ junto com funções contínuas $\phi_{i}: X_{B} \rightarrow X_{B \cup\{i\}}$ para todo $i \in\langle n\rangle$ e $B \subseteq\langle n\rangle$ tais que os espaços $X_{B}$ e as funções $\phi_{i}$ formam um $n$-cubo comutativo.

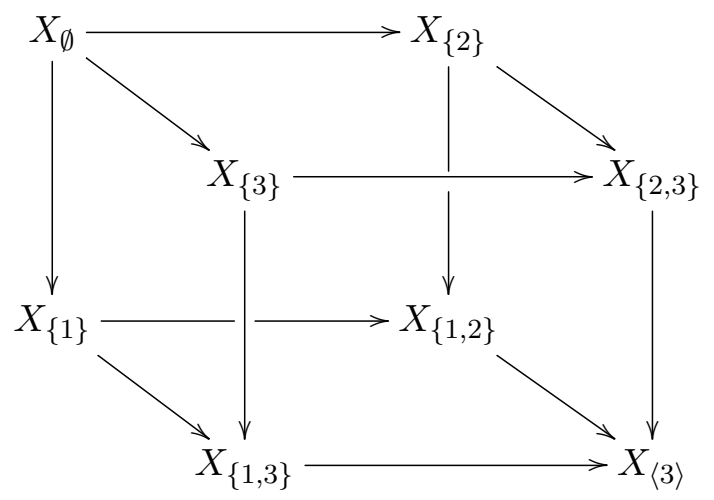

Figura 2.1: 3-cubos de espaços.

Note a inversão no sentido dos morfismos em relação aos conjuntos indexantes, comparado com os n-cubos de grupos no capítulo anterior. O motivo dessa inversão se tornará explícito na seção 3.6 aonde daremos a construção do $n$-cubo cruzado de grupos fundamental de um $n$-cubo de fibrações. Isso ocorre essencialmente pois, assim como no caso dos módulos cruzados fundamentais de uma fibração, o $n$-cubo cruzado de grupos é dado pelos grupos fundamentais de certas fibras.

Um exemplo de $n$-cubo de espaços pode ser construído a partir de uma $(n+1)$-tupla de espaços pontuados $\mathcal{X}=\left(X_{\langle n\rangle} ; X_{1}, \ldots, X_{n}\right)$, aonde $\left\{X_{i}\right\}_{i \in\langle n\rangle}$ são subespaços de $X_{\langle n\rangle}$ com o mesmo ponto base, definindo $X_{B}=\underset{i \notin B}{\cap} X_{i}$ para $B \subset\langle n\rangle$ e todas as aplicações como inclusões.

Definição 2.1.2. Um morfismo de n-cubos de espaços $f: X \rightarrow Y$ é uma família de funções contínuas $f_{B}: X_{B} \rightarrow Y_{B}$, para $B \subseteq\langle n\rangle$, que comutam com as funções $\phi_{i}$.

Nos focaremos em um tipo específico de $n$-cubo de espaços, os $n$-cubos fibrantes. A definição desta estrutura leva em conta o conceito de limite homotópico, que pode ser descrito da seguinte maneira. Todo diagrama em Top pode ser canonicamente substituído por um diagrama do mesmo formato cujos objetos são homotipicamente equivalentes aos objetos do diagrama original e os morfismos são todos fibrações de Hurewicz [Lur09, Ap. A.2]. O limite homotópico de um diagrama é o limite desse novo diagrama composto por fibrações. O motivo que limites homotópicos são usados aqui ao invés do conceito usual de limites, como descrito em [Mac98], é que limites não satisfazem a propriedade de invariância de homotopia, enquanto os limites homotópicos satisfazem. Em outras palavras dada uma categoria pequena $\mathcal{D}$, dois funtores $F, F^{\prime}: \mathcal{D} \rightarrow$ Top e uma transformação natural $\eta: F \Rightarrow F^{\prime}$ tal que cada $\eta_{d}: F(d) \rightarrow F^{\prime}(d)$ é uma equivalência homotópica não é necessariamente verdade que o morfismo induzido

$$
\lim _{d \in \mathcal{D}} F(d) \rightarrow \lim _{d \in \mathcal{D}} F^{\prime}(d)
$$


é uma equivalência homotópica, porém é verdade que o morfismo

$$
\underset{d \in \mathcal{D}}{\operatorname{holim}} F(d) \rightarrow \underset{d \in \mathcal{D}}{\operatorname{holim}} F^{\prime}(d)
$$

é uma equivalência homotópica. Vemos portanto que limites homotópicos tem um comportamento bom para se fazer teoria de homotopia. Mais detalhes sobre limites homotópicos podem ser encontrados no livro de Hirschhorn [Hir09].

Definição 2.1.3. Um n-cubo de espaços X é um n-cubo fibrante se as aplicações canônicas

$$
p_{B}: X_{B} \rightarrow \operatorname{holim}_{S \supset B} X_{S}
$$

são fibrações de Hurewicz.

Todo $n$-cubo de espaços que não satisfaz essa propriedade está canonicamente associado a um que satisfaz e cujos espaços são homotopicamente equivalentes aos espaços do $n$-cubo original, como está demonstrado em [EH76, Cap. 3]. Note também que, como fibrações são fechadas por pullbacks e composições, todas as aplicações em um n-cubo fibrante são fibrações.

Definimos agora o conceito de $n$-cubo de fibrações, e em seguida estudaremos sua relação com a construção anterior.

Definição 2.1.4. Um n-cubo de fibrações $\bar{X}$, é um diagrama comutativo de espaços composto de espaços $\bar{X}_{A, B} \operatorname{com} A, B \subseteq\langle n\rangle$ e $A \cap B=\emptyset$ e fibrações

$$
\bar{X}_{A \cup\{i\}, B} \rightarrow \bar{X}_{A, B} \rightarrow \bar{X}_{A, B \cup\{i\}}, A \cap B=\emptyset, i \notin A \cup B,
$$

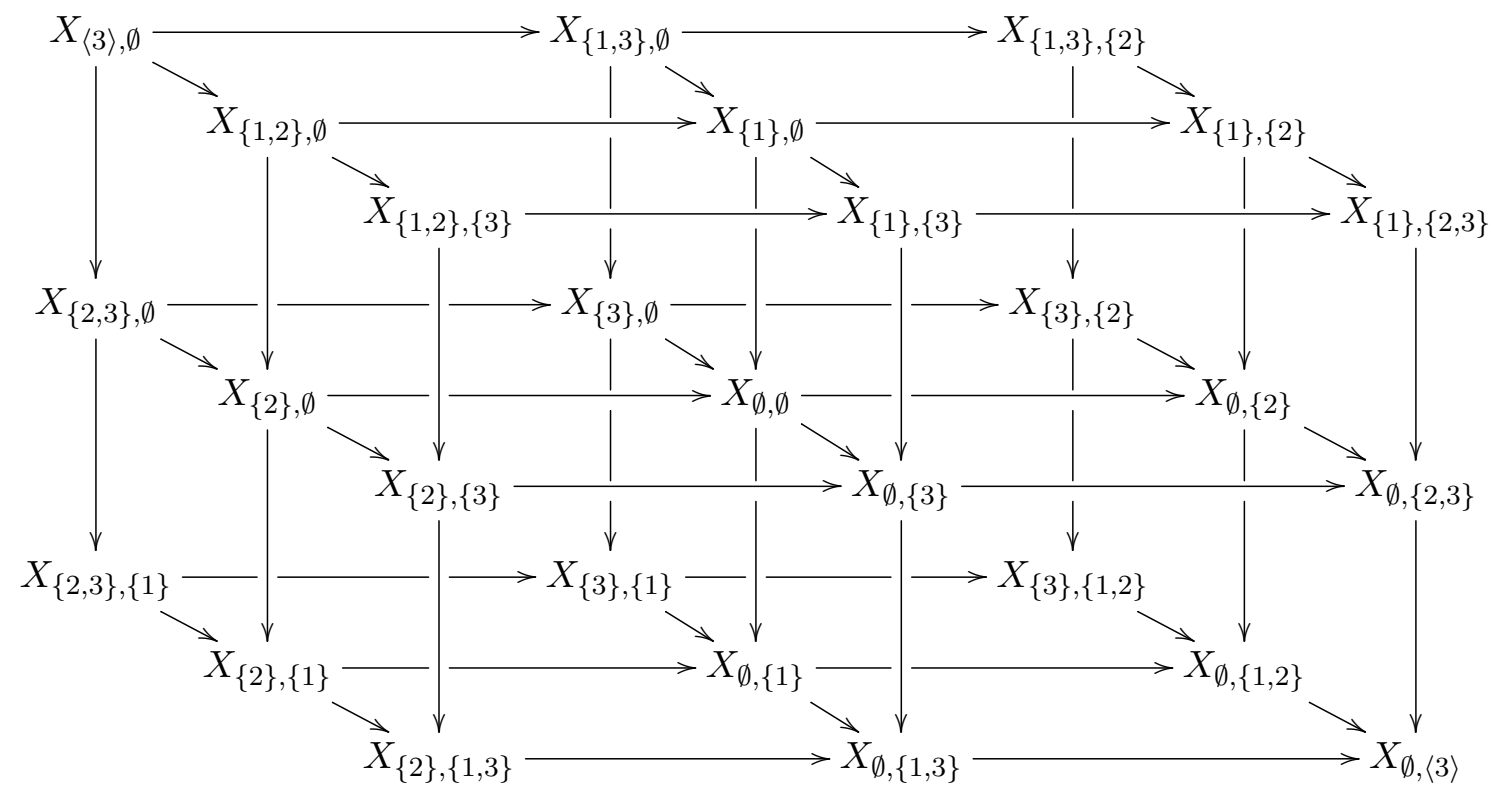

Figura 2.2: 3-cubo de fibrações.

Como intercambiaremos entre as duas categorias mencionadas com frequência, adotaremos a convenção que quando estivermos falando de $n$-cubos de fibrações usaremos notações com um traço em cima, por exemplo $\bar{X}$, e quando estivermos falando de $n$-cubos fibrantes usaremos notações sem o traço em cima, por exemplo $X$. 
Como veremos na seção 3.6, o $n$-cubo cruzado de grupos fundamental será construído usando o grupo fundamental dos espaços $\bar{X}_{A, \emptyset}$, o que sugere a inversão dos morfismos em relação aos subgrupos indexantes mencionada antes.

Definição 2.1.5. Um morfismo de n-cubos de fibrações $f: \bar{X} \rightarrow \bar{Y}$ é uma família de funções contínuas $f_{A, B}: \bar{X}_{A, B} \rightarrow \bar{Y}_{A, B}$ para $A, B \subseteq\langle n\rangle$ e $A \cap B=\emptyset$ que comutam com os morfismos dos $n$-cubos de fibração.

Para provar nessa seção a equivalência entre a categoria dos n-cubos de fibrações da definição 2.1.4 e a categoria dos $n$-cubos fibrantes da definição $\mathbf{2 . 1 . 3}$, assim como a equivalência demonstrada na próxima seção entre a categoria homotópica dos $n$-tipos homotópicos com uma localização de uma certa subcategoria da categoria dos $n$-cubos fibrantes, precisaremos definir alguns tipos de equivalência entre $n$-cubos de fibrações.

Definição 2.1.6. Um morfismo de $n$-cubos de fibrações $f$ é uma equivalência se cada $f_{A, B}$ é uma equivalência homotópica fraca. Chamamos $f$ de uma equivalência de espaço base se $f_{\emptyset,\langle n\rangle}$ for uma equivalência homotópica fraca. Dizemos que $\bar{X}$ e $\bar{Y}$ são equivalentes se existir uma equivalência entre eles, e que são equivalentes por espaço base se houver uma equivalência por espaços base entre eles.

Também será importante definir um conceito de conectividade para os $n$-cubos de fibrações, já que isso será uma hipótese central no teorema generalizado de Seifert-van Kampen.

Definição 2.1.7. Um $n$-cubos de fibrações é conexo se todos os espaços $\bar{X}_{A, B}$ são conexos por caminho. Uma $(n+1)$-tupla de espaços $\chi$ é conexa se o $n$-cubo de fibrações associado for conexo.

Note que podemos usar as sequências exatas das fibrações para mostrar que basta que os espaços $\bar{X}_{A, B}$ para $A \cup B=\langle n\rangle$ sejam conexos para que $\bar{X}$ seja conexo.

A equivalência das duas estruturas definidas é dada pelos seguintes teoremas, cujas demonstrações contém as construções do $n$-cubo de fibrações associados a um $n$-cubo fibrante e o $n$-cubo fibrante associado a um $n$-cubo de fibrações.

Teorema 2.1.8. Um n-cubo fibrante $X$ estende-se a um n-cubo de fibrações $\bar{X}$ tal que $\bar{X}_{\emptyset, B}=X_{B}$ para todo $B \subseteq\langle n\rangle$. Além disso $\bar{X}$ é conexo se, e somente se, as fibrações $p_{B}$ tem fibra conexa por caminhos para todo $B \subseteq\langle n\rangle$.

Teorema 2.1.9. Todo $n$-cubo de fibrações é equivalente a uma extensão de um n-cubo fibrante como no teorema 2.1.8

Para provar o teorema 2.1.8 precisaremos estabelecer algumas definições e provar dois lemas. Chamamos uma família $\Phi$ de subconjuntos de $\langle n\rangle$ fechada por união se $S, T \in \Phi$ implica $S \cup T \in \Phi$. Se $\Phi$ e $\Psi$ são famílias fechadas por união, então denotaremos $\Phi \leq \Psi$ se todo membro de $\Psi$ conter um membro de $\Phi$. O importante de se ter em mente é que nesse caso temos para um $n$-cubo de espaços $X$ uma aplicação

$$
q_{\Phi, \Psi}: \operatorname{holim}_{S \in \Phi} X_{S} \rightarrow \operatorname{holim}_{S \in \Psi} X_{S}
$$


Lema 2.1.10. Seja $X$ um n-cubo de espaços e sejam $\Phi$ e $\Psi$ famílias fechadas por união de subconjuntos de $\langle n\rangle$. Então existe um pull-back

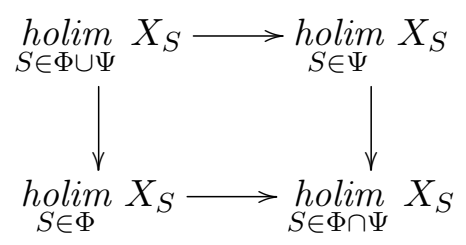

Demonstração: As funções do pullback são dadas por funções do tipo mencionada no comentário antes do enunciado do lema. A propriedade universal é uma consequência direta da definição de limite homotópico.

Lema 2.1.11. Seja $X$ um n-cubo fibrante e sejam $\Phi$ e $\Psi$ famílias fechadas por união de subconjuntos de $\langle n\rangle$ tais que $\Phi \leq \Psi$. Então $q_{\Phi, \Psi}$ é uma fibração. Se as fibrações $p_{B}$ tem fibra conexa por caminhos, então $q_{\Phi, \Psi}$ tem fibra conexa por caminhos.

Demonstração: O limite $\operatorname{holim}_{S \in \Phi} X_{S}$ não se altera se trocarmos $\Phi$ por

$$
\{T \subseteq\langle n\rangle \mid T \text { contém algum membro de } \Phi\}
$$

Podemos portanto assumir que todo subconjunto de $\langle n\rangle$ contendo algum membro de $\Phi$ está também contido em $\Phi$, e analogamente para $\Psi$. A hipótese $\Phi \leq \Psi$ se torna então $\Phi \supseteq \Psi$. Liste os elementos de $\Phi \backslash \Psi$ como $S(1), \ldots, S(k)$ de tal forma que se $S(i) \subset S(j)$ então $i<j$, e defina recursivamente $\Omega(0)=\Phi$ e $\Omega(i)=\Omega(i-1) \backslash\{S(i)\}$ para $1 \leq i \leq k$. Então $q_{\Phi, \Psi}$ é a composição dos $q_{\Omega(i-1), \Omega(i)}$ e o lema 2.1.10 nos dá um pullback

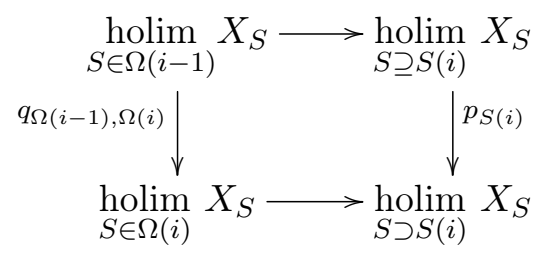

Como as classes de fibrações e as de fibrações com fibras conexas por caminhos são ambas fechada por pullbacks e composições, obtemos o resultado desejado.

Demonstração de 2.1.8: Seja $X$ um n-cubo fibrante. Definimos $\bar{X}_{A, B}, \operatorname{com} A \cap B=\emptyset$, como a fibra de

$$
X_{B} \rightarrow \operatorname{holim}_{\substack{S \supseteq B \\ S \cap A \neq \emptyset}} X_{S}
$$

Temos assim um diagrama comutativo com $\bar{X}_{\emptyset, *}=X$ e $\bar{X}_{A \cup\{i\}, B} \rightarrow \bar{X}_{A, B}$ a fibra de $\bar{X}_{A, B} \rightarrow$ $\bar{X}_{A, B \cup\{i\}}$ para $i \notin A \cup B$. Além disso os espaços $\bar{X}_{\langle n\rangle \backslash B, B}$ são as fibras de $p_{B}$, logo um $n$-cubo de fibrações $\bar{X}$ será conexo se, e somente se, os $p_{B}$ tiverem fibras conexas por caminhos. Resta mostrar que as aplicações $\bar{X}_{A, B} \rightarrow \bar{X}_{A, B \cup\{i\}}$, para $i \notin A \cup B$, são de fato fibrações.

Sejam $\Phi$ e $\Psi$ famílias fechadas por união de subconjuntos de $\langle n\rangle$ dadas por

$$
\Phi=\{S \mid S \supseteq B \cup\{i\}\}, \quad \Psi=\{S \mid S \supseteq B, S \cap A \neq \emptyset\}
$$


Existe então o diagrama comutativo

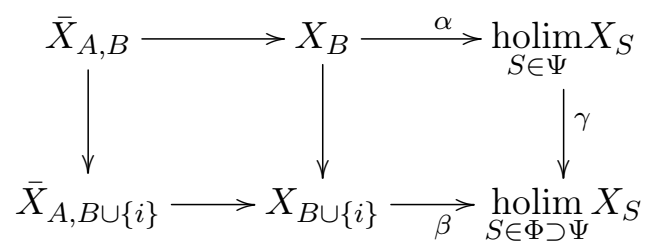

$\operatorname{com} \bar{X}_{A, B}$ e $\bar{X}_{A, B \cup\{i\}}$ as fibras de $\alpha$ e $\beta$, logo se $P$ é o pullback de $\beta$ e de $\gamma$ existe um pullback

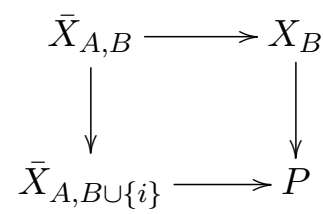

Mas $X_{B \cup\{i\}}=\operatorname{holim}_{S \in \Phi} X_{S}$, logo $P=\operatorname{holim}_{S \in \Phi \cup \Psi} X_{S}$ pelo lema 2.1.10, e assim $X_{B} \rightarrow P$ é uma fibração pelo lema 2.1.11. Portanto $\bar{X}_{A, B} \rightarrow \bar{X}_{A, B \cup\{i\}}$ é uma fibração já que pullbacks preservam fibrações. Isso completa a demonstração.

Demonstração de 2.1.9: Dado um $n$-cubo de fibrações $\bar{X}$ construímos um $n$-cubo fibrante $Y$ e um morfismo $f: \bar{X}_{\emptyset, *} \rightarrow Y$ definindo $Y_{\langle n\rangle}=X_{\emptyset,\langle n\rangle}$, com $f_{\langle n\rangle}=\mathbb{1}$ e recursivamente fatorando

$$
\bar{X}_{\emptyset, B} \rightarrow \operatorname{holim}_{S \supset B} \bar{X}_{\emptyset, S} \stackrel{\operatorname{holim}_{S \supset B}}{\longrightarrow} \operatorname{holim}_{S \supset B} Y_{S}
$$

em uma equivalência homotópica, que definimos como a função $f_{B}: \bar{X}_{\emptyset, B} \rightarrow Y_{B}$, e uma fibração, que definimos como a $p_{B}: Y_{B} \rightarrow \underset{S \supset B}{\operatorname{holim}} Y_{S}$. Pela construção do $n$-cubo de fibrações associado a $Y$ vista na demonstração anterior, temos que $\bar{X}$ e $\bar{Y}$ são equivalentes.

Como corolário direto dos teoremas 2.1.8 e 2.1.9 temos a equivalência que queríamos.

Corolario 2.1.12. A categoria dos n-cubos fibrantes e a categoria dos n-cubos de fibrações são equivalentes.

No caso da $(n+1)$-tupla pontuada $\mathcal{X}=\left(X_{\langle n\rangle} ; X_{1}, \ldots, X_{n}\right)$ temos que o $n$-cubo de fibrações associado é dado por $\bar{X}$ aonde para $A=\emptyset$ temos

$$
\bar{X}_{\emptyset, B}=\left\{\gamma \in X_{\langle n\rangle} \mid \gamma(1) \in X_{B}\right\}
$$

e para $A \neq \emptyset$ temos

$$
\begin{aligned}
\bar{X}_{A, B}=\left\{\gamma \in\left(X_{A \cup B}\right)^{I^{A}} \mid\right. & \gamma\left(\left(t_{i}\right)_{i \in A}\right)=x_{0} \text { se } t_{j}=0 \text { para algum } j \in A \\
& \text { e } \left.\gamma\left(\left(t_{i}\right)_{i \in A}\right) \in X_{(A \cup B) \backslash\{j\}} \text { se } t_{j}=1 \text { para algum } j \in A\right\},
\end{aligned}
$$

cujo ponto base é a função que leva todo ponto de $I^{A}$ no ponto base de $\mathcal{X}$.

Dizemos que uma $(n+1)$-tupla pontuada $\mathcal{X}=\left(X_{\langle n\rangle} ; X_{1}, \ldots, X_{n}\right)$ é conexa se seu $n$-cubo de fibrações associado é conexo.

\subsection{Equivalência de $n$-cubos de Eilenberg-MacLane e $(n+1)$-tipos homotópicos}

Uma subcategoria importante da categoria dos n-cubos de fibrações é dado pela definição abaixo. 
Definição 2.2.1. Um $n$-cubo de fibrações $\bar{X}$ é um n-cubo de Eilenberg-MacLane se ele for conexo e todos os espaços $\bar{X}_{A, \emptyset}$ forem espaços de Eilenberg-Maclane do tipo $K(\pi, 1)$.

Mostraremos agora como a localização da subcategoria dos n-cubos de Eilenberg-MacLane, obtida invertendo as equivalências de espaços bases, é equivalente à categoria homotópica dos $(n+1)$ tipos homotópicos, lembrando que um espaço topológico $Z$ é um $n$-tipo homotópico se $\pi_{i}(Z)=0$ para todo $i>n$. Para tal, será mais pratico usar a categoria equivalente dos $n$-cubos fibrantes.

Definição 2.2.2. Um n-cubo fibrante é conexo ou de Eilenberg-MacLane se seu $n$-cubo de fibrações associado o for. Um morfismo de $n$-cubos fibrantes é uma equivalência ou equivalência de espaço base se o morfismo de $n$-cubos de fibrações associado o for.

Antes de demonstrar a equivalência dessa seção precisamos primeiro mostrar que existe uma localização da categoria de $n$-cubos fibrantes associada ao conjunto das equivalências de espaço base. Lembremos a propriedade universal das localizações.

Definição 2.2.3. Dada uma categoria $\mathcal{C}$ e um subconjunto $W$ dos morfismos de $\mathcal{C}, \mathcal{C} W^{-1}$ é uma localização de $\mathcal{C}$ em $W$ se existe um funtor $i: \mathcal{C} \rightarrow \mathcal{C} W^{-1}$ tal que um funtor $F: \mathcal{C} \rightarrow \mathcal{D}$ se fatora por $i$ se, e somente se, $F$ manda todos os elementos de $W$ em isomorfismos.

Lema 2.2.4. Existe uma localização da categoria de n-cubos fibrantes associada ao conjunto das equivalências de espaço base.

Demonstração Dado o resultado [GZ67, prop. 2.4] basta mostrar que as equivalências de espaços bases admitem um calculo de frações à direita. Denote o conjunto das equivalências de espaços base por $W$. Claramente $W$ contém as identidades e é fechada por composição.

Para todo diagrama $Y \stackrel{f}{\rightarrow} Z \stackrel{v}{\leftarrow} X$ com $v \in W$ e $f$ um morfismo qualquer podemos definir $V$ o $n$-cubo fibrante associado a $(n+1)$-tupla pontuada $\left(Y_{\langle n\rangle} ; *, \ldots, *\right)$, ou seja tomamos o ponto base como os subespaços. Definimos $f^{\prime}: V \rightarrow X$ e $v^{\prime}: V \rightarrow Y \operatorname{com} f_{\langle n\rangle}^{\prime}=f_{\langle n\rangle}, v_{\langle n\rangle}^{\prime}=\mathbb{1}_{Y_{\langle n\rangle}}$ e ambas sendo a função constante nas funções indexadas pelos outros subconjuntos $B \subset\langle n\rangle$. Temos então que $v^{\prime} \in W$ e o diagrama abaixo comuta.

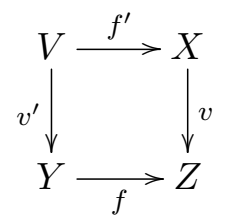

Para todo diagrama $X \underset{g}{\stackrel{f}{\rightrightarrows}} Y \stackrel{v}{\rightarrow} Z$ com $v \in W$ e $v \circ f=v \circ g$ podemos tomar $V$ como o $n$-cubo fibrante associado a $(n+1)$-tupla pontuada $\left(X_{\langle n\rangle} ; *, \ldots, *\right)$ como antes e $v^{\prime}: V \rightarrow X$ com $v_{\langle n\rangle}^{\prime}=\mathbb{1}_{X\langle n\rangle}$ e sendo a função constante nos outros índices $B \subset\langle n\rangle$. Temos então que $v^{\prime} \in W$ e $f \circ v^{\prime}=g \circ v^{\prime}$.

Uma construção explicita da localização de uma categoria associada a um subconjunto pode ser encontrada no livro de Gabriel e Zisman [GZ67].

Note que pelos resultados da seção anterior, podemos trocar $n$-cubos fibrantes por $n$-cubos de fibrações no teorema abaixo. 
Teorema 2.2.5. A localização da categoria dos n-cubos fibrantes de Eilenberg-MacLane obtida invertendo formalmente as equivalência de espaços base é equivalente à te a categoria homotópica dos $(n+1)$-tipos homotópicos conexos por caminhos

Demonstração: Começamos construindo os funtores

$$
\begin{aligned}
& F:\{n \text {-cubos fibrantes }\} \rightarrow\{\text { espaços topológicos }\} \\
& G:\{\text { espaços topológicos }\} \rightarrow\{n \text {-cubos fibrantes }\}
\end{aligned}
$$

O funtor $F$ simplesmente toma um $n$-cubo fibrante $X$ e o leva para $F(X)=X_{\langle n\rangle}$.

Para um espaço $Z$ definimos $G(Z)$ recursivamente. Definimos $G(Z)_{\langle n\rangle}=Z$. Agora para $B \subseteq\langle n\rangle$ tome $V_{B}$ a realização geométrica do 1-esqueleto do complexo singular de $\underset{S \supset B}{\operatorname{holim}} G(Z)_{S}$. Definimos então $G(Z)_{B}$ como o espaço obtido fatorizando a aplicação de avaliação $a: V_{B} \rightarrow \underset{S \supset B}{\operatorname{holim} G(Z)_{S}}$ em uma equivalência homotópica $V_{B} \rightarrow G(Z)_{B}$, e definimos $p_{B}$ como a fibração $p_{B}: G(Z)_{B} \rightarrow$ $\operatorname{holim}_{S \supset B} G(Z)_{S}$ dessa decomposição.

Temos que os $p_{B}$ induzem sobrejeções em $\pi_{1}$ e injeções em $\pi_{0}$, logo sua fibra é conexa por caminhos e portanto, pelo teorema 2.1.8, se $Z$ for conexo por caminhos então $G(Z)$ é conexo. Além disso como $G(Z)_{B}$ são do tipo homotópico de 1-complexos para $B \neq\langle n\rangle$ temos que eles são espaços de Eilenberg-MacLane do tipo $K(\pi, 1)$.

Claramente $F$ leva equivalências de espaço base para equivalências homotópicas fracas, $G$ leva equivalências homotópicas fracas para equivalências de espaço base, e $F \circ G=\mathbb{1}$. Mostramos agora que $G \circ F$ é naturalmente equivalente por espaço base à identidade.

Para isso primeiro definimos um funtor

$$
H:\{n \text {-cubos fibrantes }\} \rightarrow\{2 n \text {-cubos fibrantes }\}
$$

aonde $H(X)$ é indexado por pares de subconjunto de $\langle n\rangle$ não necessariamente disjuntos. Definimos que

$$
H(X)_{B,\langle n\rangle}=X_{B} \quad \text { e } \quad H(X)_{\langle n\rangle, B}=G \circ F(X)_{B}
$$

Temos portanto que nos casos em que $A=\langle n\rangle$ ou $B=\langle n\rangle$ os espaços e as fibrações abaixo estão bem definidos

$$
p_{A, B}: H(X)_{A, B} \rightarrow \operatorname{holim}_{\substack{T \supset A, U \supset B \\(T, U) \neq(A, B)}} H(X)_{T, U}
$$

Para os outros valores de $A$ e $B$ repetimos a construção recursiva de $G$ de forma que $H(X)_{D, E}$ tenham o tipo homotópico de 1-complexos e que os $p_{D, E}$ tenham fibras conexas por caminho. Definimos então um funtor "diagonal" $D$ de $n$-cubos fibrantes tal que

$$
D(X)_{B}=H(X)_{B, B}
$$

Essa construção então induz equivalências de espaço base $X \leftarrow D(X) \rightarrow G \circ F(X)$ fazendo $X$ e $G \circ F(X)$ naturalmente equivalentes por espaço base.

Falta mostrar que os funtores $F, G$ e $D$ se restringem às subcategorias de interesse de forma adequada.

Seja $X$ um $n$-cubo fibrante de Eilenberg-MacLane. Como $X$ é conexo $F(X)=X_{\langle n\rangle}$ é conexo por caminhos. Se $\bar{X}$ é a extensão de $X$ em um $n$-cubo de fibrações, então $\pi_{i}\left(\bar{X}_{A, \emptyset}\right)=0$, para $i>1$, e por indução no tamanho de $B$ obtemos usando a sequencia exata das fibrações que $\pi_{i}\left(\bar{X}_{A, B}\right)=0$, 
para $i>|B|+1$. Em particular $\pi_{i}(F(X))=\pi_{i}\left(\bar{X}_{\emptyset,\langle n\rangle}\right)=0$ para $i>n+1$, ou seja $F(X)$ é um $(n+1)$-tipo homotópico.

Seja $Z$ um $(n+1)$-tipo homotópico conexo por caminhos. A fibração $p_{\langle n\rangle}$ em $G(Z)$ tem fibra conexa por caminhos $Z$ e como vimos os outros $p_{B}$ também tem fibras conexas por caminhos, logo pelo teorema 2.1.8 $G(Z)$ é conexo. Como no $n$-cubo de fibrações $G \overline{(Z)}$ os espaços $G \overline{(Z})_{\emptyset, B}=G(Z)_{B}$, para $B \neq\langle n\rangle$, são do tipo $K(\pi, 1)$ segue por indução no tamanho de $A$ que pelas sequencias exatas das fibrações todos os $G \overline{(Z})_{A, B}$ são do tipo $K(\pi, 1)$ para $A \cup B \neq\langle n\rangle$, em particular $\left.G \overline{(Z)}\right)_{A, \emptyset}$ são do tipo $K(\pi, 1)$ para $A \neq\langle n\rangle$. Uma indução no tamanho de $A$ agora nos mostra que se $A \cup B=\langle n\rangle$ então, pelas sequencias exatas das fibrações, $\left.\pi_{i}(G \overline{(Z})_{A, B}\right)=0$ para $i>n-|A|+1$. Em particular

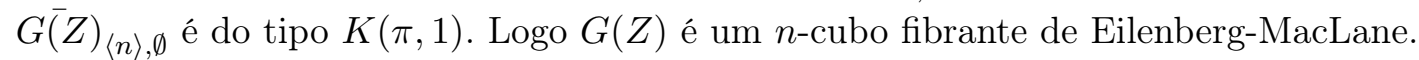

Agora tomemos $X$ um $n$-cubo fibrante de Eilenberg-MacLane novamente. Por hipótese $X$ é conexo e pelos dois últimos parágrafos temos $G \circ F(X)$ conexo. Como $H(X)$ é obtido com o mesmo método que $G(Z)$ acima o mesmo argumento mostra que $H(X)$ é conexo. O lema 2.1.11 implica que os $H(X)_{B, B} \rightarrow \operatorname{holim}_{S \supset B} H(X)_{S, S}$ tem fibra conexa por caminhos, o que é equivalente a dizer que $p_{B}: D(X)_{B} \rightarrow \underset{S \supset B}{\operatorname{holim} D} D(X)_{S}$ tem fibra conexa por caminhos. Portanto o mesmo argumento usado para $G(Z)$ mostra que $D(X)$ é um $n$-cubo fibrante de Eilenberg-MacLane. 



\section{Capítulo 3}

\section{Cat $^{n}$-grupos e $n$-cubos cruzados de grupos fundamentais}

Nesse capitulo mostraremos como as estruturas dos capítulos anteriores se relacionam. Faremos muito uso de conceitos e resultados da teoria de homotopia simplicial, então na primeira seção veremos algumas preliminares dessa teoria. Apresentaremos as definições que precisaremos e enunciamos sem demonstrações os principais resultados que usaremos. Para detalhes sobre a teoria de homotopia simplicial sugerimos o livro de Goerss e Jardine [GJ99] ou o de May [May92].

$\mathrm{Na}$ segunda seção construímos o funtor cat $^{n}$-grupo fundamental, denotado $\Pi$, da categoria dos $n$-cubo fibrante à categoria dos cat ${ }^{n}$-grupo.

Na terceira seção construiremos o funtor espaço de classificação, denotado $B$, da categoria dos cat $^{n}$-grupos à categoria dos espaços. Usando o funtor $B$ construiremos também o funtor $n$-cubo de fibrações de classificação, denotado $\mathcal{B}$, da categoria dos cat ${ }^{n}$-grupos à categoria dos $n$-cubos de fibrações, e demonstraremos que esse funtor, junto com $\Pi$, forma uma equivalência entre as categorias dos cat $^{n}$-grupos e a dos $n$-cubos de Eilenberg-MacLane.

Esse resultado, junto com o da seção 2.2, generaliza o resultado de MacLane e Whitehead que diz que todo 2-tipo homotópico é realizável como o espaço de classificação de um módulo cruzado [MW50], provado usando outros métodos (nesse trabalho eles chamam de 3-tipos homotópicos aquilo que aqui chamamos de 2-tipos homotópicos).

Dado os resultados acima podemos levantar a questão de como obter algebricamente os grupos de homotopia do $(n+1)$-tipo homotópico associado a um cat ${ }^{n}$-grupo. Na quarta seção respondemos essa questão. A partir dessa resposta obtemos um conceito de quasi-isomorfismos entre cat $^{n}$-grupos, de forma que também obtemos nessa seção uma equivalência entre a categoria homotópica dos $(n+1)$-tipos homotópicos e a localização da categoria dos cat ${ }^{n}$-grupos obtida introduzindo inversas formais dos quasi-isomorfismos.

A quinta seção é dedicada a demonstrar o teorema de Seifert-Van Kampen generalizado, que diz que o funtor $\Pi$ preserva colimites de certos diagramas de $n$-cubos fibrantes conexos, e que nesses casos o colimite preserva conectividade.

Nesse capítulo todos os resultados são referentes ao funtor cat ${ }^{n}$-grupo fundamental $\Pi$, porém a equivalência da seção 1.3 sugere a existência de um funtor $n$-cubo cruzado de grupos fundamental $\Pi$ com as mesmas propriedades. A última seção é dedicada a uma descrição direta do $n$-cubo cruzado de grupos fundamental de um $n$-cubo de fibrações. A parte mais trabalhosa é explicitar o quê dará origem às funções $h$ da definição de $n$-cubos cruzados de grupos, e veremos que elas surgem como produtos generalizados de Whitehead definidos a partir de um exemplo universal. Usaremos o funtor $\Pi$, assim como as computações de colimites no capitulo $\mathbf{1}$, nas aplicações do teorema generalizado de Seifert-van Kampen do próximo capítulo. 


\subsection{Homotopia simplicial}

Um $m$-simplexo é a generalização do conceito de triângulos e tetraedros para $m$ dimensões. Podemos definir o $m$-simplexo padrão como o subconjunto de $\mathbb{R}^{m+1}$ dado por

$$
\Delta_{m}=\left\{t_{1} e_{1}+\cdots+t_{m+1} e_{m+1} \mid t_{i}>0 \text { e } \sum_{0 \leq i \leq m} t_{i}=1\right\} .
$$

aonde $e_{i}$ é o vetor com valor 1 na coordenada $i$ e 0 em todas as outras. O $m$-simplexo padrão possui para cada $i$ tal que $0 \leq i \leq m$ uma face denotada $\partial_{i}\left(\Delta_{n}\right)$ homeomorfa ao $(m-1)$-simplexo padrão dada pelo subconjunto de $\Delta_{m}$ tal que $t_{i+1}=0$. Complexos simpliciais são espaços obtidos colando cópias dos $m$-simplexos padrões, cada uma chamada de um $m$-simplexo, identificando suas faces. Esses espaços tem propriedades combinatórias que permitem muitas aplicações à teoria de homologia. Atualmente uma construção parecida, os conjuntos simpliciais, é utilizada na teoria de homotopia. A importância dos conjuntos simpliciais é que eles servirão como intermediários entre as categorias topológicas do capítulo $\mathbf{2}$ e as categorias algébricas do capítulo $\mathbf{1}$.

Antes de definir um conjunto simplicial será preciso definir a categoria dos simplexos, que deve ser pensada como a categoria que captura as características puramente combinatórias dos $m$-simplexos.

Definição 3.1.1. A categoria dos simplexos, denotada $\Delta$, tem como objetos os conjuntos ordenados $\mathbf{m}=\{0<1<\cdots<m\}$ e como morfismos as funções $\theta: \mathbf{m} \rightarrow \mathbf{n}$ que preservam a ordem. Essa categoria tem uma apresentação tal que ela é gerada pelos morfismos

- Aplicações de bordo $\partial_{i}: \mathbf{m}-\mathbf{1} \rightarrow \mathbf{m}$ para $0 \leq i \leq m$ dada pela única injeção na qual $i \in \mathbf{m}$ não está contido na imagem;

- Aplicações de degeneração $d_{i}: \mathbf{m}+\mathbf{1} \rightarrow \mathbf{m}$ para $0 \leq i \leq m$ dada pela única sobrejeção tal que $d_{i}(i)=d_{i}(i+1)$;

e as relações são dadas pelas relações

$$
\begin{gathered}
\partial_{j} \circ \partial_{i}=\partial_{i} \circ \partial_{j-1} \quad i<j \\
d_{j} \circ d_{i}=d_{i} \circ d_{j+1} \quad i \leq j \\
d_{j} \circ \partial_{i}= \begin{cases}\partial_{i} \circ d_{j-1} & i<j \\
\mathbb{1}_{\mathbf{m}} & i=j \text { ou } i=j+1 \\
\partial_{i-1} \circ d_{j} & i>j+1\end{cases}
\end{gathered}
$$

Podemos enfim definir um objeto simplicial de uma categoria. Chamamos de conjunto simplicial um objeto simplicial da categoria dos conjuntos. Objetos simpliciais em outras categorias seguem a mesma nomenclatura, portanto podemos falar de grupos simpliciais, espaços simpliciais, etc.

Definição 3.1.2. Dada uma categoria $\mathcal{C}$ podemos definir um objeto simplicial da categoria $\mathcal{C}$ como um funtor $C_{\bullet}: \Delta^{\mathrm{op}} \rightarrow \mathcal{C}$. Mais explicitamente vai ser um diagrama com objetos $C_{m}$ para cada $m \in \mathbb{N}$ e morfismos de bordo $\partial_{i}: C_{m} \rightarrow C_{m-1}$ e morfismos de degeneração $d_{i}: C_{m} \rightarrow C_{m+1}$ para 
$0 \leq i \leq m$ que satisfazem as relações

$$
\begin{gathered}
\partial_{i} \circ \partial_{j}=\partial_{j-1} \circ \partial_{i} \quad i<j \\
d_{i} \circ d_{j}=d_{j+1} \circ d_{i} \quad i \leq j \\
\partial_{i} \circ d_{j}= \begin{cases}d_{j-1} \circ \partial_{i} & i<j \\
\mathbb{1}_{\mathbf{m}} & i=j \text { ou } i=j+1 \\
d_{j} \circ \partial_{i-1} & i>j+1\end{cases} \\
\ldots \stackrel{\Longleftrightarrow}{\Longleftrightarrow} C_{2} \rightleftarrows C_{1} \rightleftarrows C_{0}
\end{gathered}
$$

Figura 3.1: Objeto simplicial $C$. em $\mathcal{C}$.

Podemos ver a relação entre os conjuntos simpliciais e os espaços topológicos pela existência do funtor descrito a seguir, que usa a informação combinatória contida nos conjuntos simpliciais para construir espaços usando simplexos, de forma parecida à construção de complexos simpliciais.

Definição 3.1.3. Dado um conjunto simplicial $S_{\bullet}$, a sua realização geométrica $\left|S_{\bullet}\right|$ é dada pelo quociente da união disjunta

$$
\underset{m \in \mathbb{N}}{\sqcup} S_{m} \times \Delta_{m}
$$

sendo que para todo $m \in \mathbb{N}$ e cada $s \in S_{m}$ identificamos o espaço $\partial_{i}(s) \times \Delta_{m-1}$ com o espaço $s \times \partial_{i}\left(\Delta_{m}\right)$ pelo isomorfismo natural entre $\Delta_{m-1}$ e $\partial_{i}\left(\Delta_{m}\right)$.

Podemos tomar o objeto simplicial dentro de uma categoria de objetos simpliciais, e repetir esse processo um número arbitrário de vezes. Obtemos então o conceito de um objeto $n$-simplicial de uma categoria, que precisaremos para definir muitos dos funtores que aparecerão nesse capítulo.

Definição 3.1.4. Dada uma categoria $\mathcal{C}$ podemos definir um objeto $n$-simplicial da categoria $\mathcal{C}$ como um funtor $C_{\left(\bullet_{1}, \ldots, \bullet_{n}\right)}:\left(\Delta^{\text {op }}\right)^{n} \rightarrow \mathcal{C}$. Mais explicitamente vai ser um diagrama com objetos $C_{\left(m_{1}, \ldots, m_{n}\right)}$ para cada $\left(m_{1}, \ldots, m_{n}\right) \in \mathbb{N}^{n}$ e morfismos de bordo $\left.\partial_{i}^{k}: C_{(} m_{1}, \ldots, m_{k} \ldots, m_{n}\right) \rightarrow$ $C_{\left(m_{1}, \ldots, m_{k}-1 \ldots, m_{n}\right)}$ e morfismos de degeneração $d_{i}^{k}: C_{\left(m_{1}, \ldots, m_{k} \ldots, m_{n}\right)} \rightarrow C_{\left(m_{1}, \ldots, m_{k}+1 \ldots, m_{n}\right)}$ para $k \in$ $\langle n\rangle$ e $0 \leq i \leq m_{k}$ que satisfazem as relações

$$
\begin{aligned}
& \partial_{i}^{k} \circ \partial_{j}^{l}=\partial_{j}^{l} \circ \partial_{i}^{k} \quad k \neq l \\
& d_{i}^{k} \circ d_{j}^{l}=d_{j}^{l} \circ d_{i}^{k} \quad k \neq l \\
& d_{i}^{k} \circ \partial_{j}^{l}=\partial_{j}^{l} \circ d_{i}^{k} \quad k \neq l \\
& \partial_{i}^{k} \circ \partial_{j}^{k}=\partial_{j-1}^{k} \circ \partial_{i}^{k} \quad i<j \\
& d_{i}^{k} \circ d_{j}^{k}=d_{j+1}^{k} \circ d_{i}^{k} \quad i \leq j \\
& \partial_{i}^{k} \circ d_{j}^{k}= \begin{cases}d_{j-1}^{k} \circ \partial_{i}^{k} & i<j \\
\mathbb{1}_{\mathbf{m}} & i=j \text { ou } i=j+1 \\
d_{j}^{k} \circ \partial_{i-1}^{k} & i>j+1\end{cases}
\end{aligned}
$$




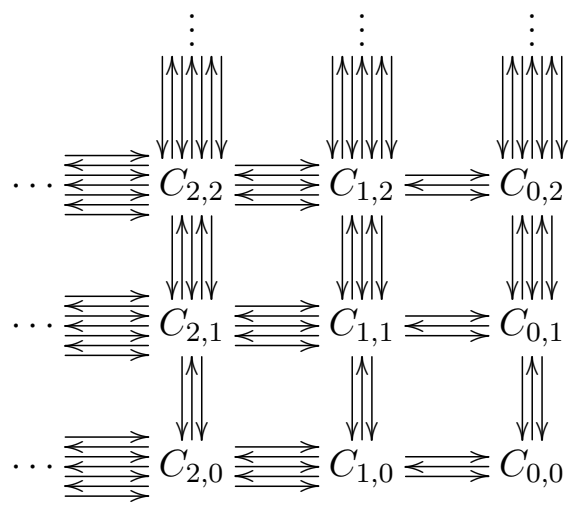

Figura 3.2: Objeto 2-simplicial $C_{\bullet_{1}, \bullet_{2}}$ em $\mathcal{C}$.

Podemos sempre passar da categoria de objetos $n$-simpliciais de uma categoria para a categoria de objetos simpliciais usando um funtor diagonal descrito a seguir. Esse funtor, junto com o funtor da definição 3.1.3 nos permite falar da realização geométrica de um conjunto $n$-simplicial.

Definição 3.1.5. Para todo $n \in \mathbb{N}$ e categoria $\mathcal{C}$ temos um funtor diagonal da categoria dos objetos $n$-simpliciais em $\mathcal{C}$ a categoria $\operatorname{dos}$ objetos simpliciais em $\mathcal{C}$, denotado $D$, aonde

$$
\begin{gathered}
D\left(C_{\left(\bullet_{1}, \ldots, \bullet_{n}\right)}\right)_{m}=C_{(m, \ldots, m)} \\
\partial_{i}=\underset{k \in\langle n\rangle}{\circ} \partial_{i}^{k} \\
d_{i}=\underset{k \in\langle n\rangle}{\circ} d_{i}^{k}
\end{gathered}
$$

Um conceito que vai ser importante para algumas definições a seguir é a de um chifre em um objeto simplicial. A ideia intuitiva é que um chifre é "como o bordo de um simplexo com uma das faces faltando".

Definição 3.1.6. Para um conjunto simplicial $S_{\bullet}, m \in \mathbb{N}$ e $0 \leq k \leq m$ um chifre $\Lambda_{m}^{k}$ de $S_{\bullet}$ é uma $m$-tupla de elementos

$$
\left(s_{0}, \ldots, s_{k-1}, s_{k+1}, \ldots, s_{m}\right) \in S_{m-1}^{m}
$$

tais que $\partial_{i}\left(s_{j}\right)=\partial_{j-1}\left(s_{i}\right)$ para $i, j \neq k$ e $i<j$.

O motivo de colocarmos "como o bordo de um simplexo com uma das faces faltando" entre aspas é que dado um chifre em um conjunto simplicial não é necessariamente verdade que existe um simplexo que o preencha. Quando existe tal simplexo para todo chifre dizemos que esse conjunto simplicial é um complexo de Kan.

Definição 3.1.7. Dado um conjunto simplicial $S_{\bullet}$, dizemos que ele é um complexo de Kan se todo chifre $\Lambda_{m}^{k}$ de $S_{\bullet}$ tem um preenchimento, ou seja se existe um elemento $s \in S_{m}$ tal que $\partial_{i}(s)=s_{i}$.

A importância desse conceito vem do fato que para todo complexo de Kan $S_{\bullet}$ é possível definir combinatoriamente grupos $\pi_{n}\left(S_{\bullet}\right)$ de forma que temos o seguinte teorema.

Teorema 3.1.8. Para todo complexo de Kan $S_{\bullet}$ temos que $\pi_{n}\left(S_{\bullet}\right) \cong \pi_{n}\left(\left|S_{\bullet}\right|\right)$.

A descrição desses grupos de homotopia não é trivial, e pode ser encontrada na literatura recomendada. Felizmente há uma descrição mais direta dos grupos de homotopia de complexos de 
Kan que surgem a partir de grupos simpliciais, e é essa descrição que precisaremos no presente trabalho. Primeiro, precisaremos da definição do nervo de uma categoria, que nos dá um conjunto simplicial a partir de uma categoria.

Definição 3.1.9. O nervo de uma categoria $\mathcal{C}$ cujo conjunto de objetos é denotado $\mathcal{C}_{0}$ e o de morfismos por $\mathcal{C}_{1}$, é o conjunto simplicial denotado por $\mathcal{N}(\mathcal{C})$ • aonde

$$
\begin{gathered}
\mathcal{N}(\mathcal{C})_{0}=\mathcal{C}_{0} \\
\mathcal{N}(\mathcal{C})_{1}=\mathcal{C}_{1} \\
\mathcal{N}(\mathcal{C})_{k}=\overbrace{\mathcal{C}_{1} \times \mathcal{C}_{0} \cdots \times \mathcal{C}_{0} \mathcal{C}_{1}}^{k \text { vezes }}
\end{gathered}
$$

para $k>1$, sendo que $\times_{\mathcal{C}_{0}}$ é o pullback das funções origem e destino.

A ideia aqui é que os simplexos de dimensão $k$ são dados por uma sequencia $\left(f_{1}, \ldots, f_{k}\right)$ de $k$ morfismos componíveis. As funções de face $\phi_{i}$ são dadas compondo o $i$-ésimo morfismo com o $(i+1)$-ésimo para $1 \leq i \leq k-1$, e $\phi_{0}$ e $\phi_{k}$ são dados deletando o primeiro e o último morfismo respectivamente. As funções de degeneração $\delta_{i}$ são dadas colocando um morfismo identidade na $(i+1)$-ésima posição para $0 \leq i \leq k$

Como todo grupo pode ser considerado uma categoria (mais precisamente um grupoide) com apenas um objeto podemos definir para um grupo $n$-simplicial $G_{\bullet}$ o seu nervo $\mathcal{N}\left(G_{\bullet}\right)$ como sendo o conjunto $(n+1)$-simplicial obtido tomando o nervo do grupo $G_{\left(\bullet_{1}, \ldots, \bullet_{n}\right)}$ para cada índice.

Teorema 3.1.10. Dado um grupo n-simplicial $G_{\left(\bullet_{1}, \ldots, \bullet_{n}\right)}$, o conjunto simplicial $D \circ \mathcal{N}\left(G_{\left(\bullet_{1}, \ldots, \bullet_{n}\right)}\right)$ • é um complexo de Kan.

Dado um grupo simplicial podemos obter um complexo de grupos não necessariamente abelianos tal que as imagens das aplicações de bordo são normais no kernel da próxima aplicação de bordo, e portanto podemos falar da homologia de tal complexo.

Definição 3.1.11. Seja $G_{\bullet}$ um grupo simplicial. O seu complexo de Moore é um complexo de grupos (não necessariamente abelianos) denotado $\left((N G) \bullet, \delta_{\bullet}\right)$ com

$$
(N G)_{m}=\underset{1 \leq i \leq m}{\cap} \operatorname{Ker} \partial_{i}
$$

e com as aplicações de bordo $\delta_{\bullet}$ dadas por

$$
\delta_{m}=\partial_{0} \Upsilon_{(N G)_{m}}:(N G)_{m} \rightarrow(N G)_{m-1}
$$

O próximo resultado, junto com o teorema 3.1.8, nos dá que essa descrição dos grupos de homologia de um grupo simplicial implica na descrição dos grupos de homotopia da realização geométrica de $\left(D \circ \mathcal{N}\left(G_{\left(\bullet_{1}, \ldots, \bullet_{n}\right)}\right)\right)$ •

Teorema 3.1.12. Seja $G_{\left(\bullet_{1}, \ldots, \bullet_{n}\right)}$ um grupo $n$-simplicial. Então para $k \geq 1$ temos

$$
\pi_{k}\left(D \circ \mathcal{N}\left(G_{\left(\bullet_{1}, \ldots, \bullet_{n}\right)}\right)\right)=H_{k-1}\left(\left(N \circ D\left(G_{\left(\bullet_{1}, \ldots, \bullet_{n}\right)}\right)\right) \bullet .\right.
$$


Como vimos na introdução e no capítulo anterior, o conceito de fibrações de Hurewicz tem um papel central na teoria apresentada nesse trabalho. Existe um conceito combinatório de fibrações entre conjuntos simpliciais dada pelas fibrações de Kan.

Definição 3.1.13. Um morfismo de conjuntos simpliciais $f: S_{\bullet} \rightarrow T_{\bullet}$ é uma fibração de Kan se para todo chifre $\Lambda_{m}^{k}$ cuja imagem por $f$ tem um preenchimento $t$ em $T_{\bullet}$ existe um preenchimento $s$ em $S_{\text {. de }} \Lambda_{m}^{k}$ tal que $f(s)=t$. Ou seja se para todo chifre composto pelos simplexos $s_{0}, \ldots, s_{k-1}, s_{k+1}, \ldots, s_{m} \in S_{m-1}$ e $t \in T_{m}$ tais que $\partial_{i}(t)=f\left(s_{i}\right)$, então existe $s \in S_{m}$ tal que $\partial_{i}(s)=s_{i}$ e $f(s)=t$

Esse conceito nos será útil dado o seguinte resultado de Quilen.

Teorema 3.1.14. A realização geométrica leva fibrações de Kan em fibrações de Hurewicz.

Esse resultado nos permitirá construir fibrações a partir de estruturas algébricas.

\subsection{Cat $^{n}$-grupo cruzado fundamental de um $n$-cubo fibrante}

Veremos agora a construção do cat ${ }^{n}$-grupo cruzado fundamental de um $n$-cubo fibrante, descrito pela primeira vez por Loday em [Lod82]. Precisaremos ver como obter a partir de uma fibração um espaço simplicial. Dada uma fibração $f: W \rightarrow Z$ podemos tomar para cada $m \in \mathbb{N}$ o limite do diagrama que contém o espaço $Z, m+1$ cópias de $W$ e $m+1$ cópias de $f$ unindo cada cópia de $W$ em $Z$. Esse limite é dado pelo subconjunto de $W^{m+1}$ dado pelos $\left(w_{0}, \ldots, w_{m}\right) \in W^{m+1}$ tais que $f\left(w_{i}\right)=f\left(w_{j}\right)$ para todo $0 \leq i, j \leq m$. Denotamos esse espaço $E_{m}^{f}$. Podemos dar a esses espaços a estrutura de um espaço simplicial tomando como aplicações de bordo $\partial_{i}$ e degeneração $d_{i}$ como

$$
\begin{gathered}
\partial_{i}\left(w_{0}, \ldots, w_{m}\right)=\left(w_{0}, \ldots, w_{i-1}, w_{i+1}, \ldots, w_{m}\right) \\
d_{i}\left(w_{0}, \ldots, w_{m}\right)=\left(w_{0}, \ldots, w_{i-1}, w_{i}, w_{i}, w_{i+1}, \ldots, w_{m}\right)
\end{gathered}
$$

Denotamos o funtor que vai da categoria de fibrações a categoria dos espaços simpliciais descrito acima de $\mathscr{S}$.

Generalizaremos essa construção e mostraremos como obter um espaço $n$-simplicial a partir de um $n$-cubo fibrante. Dado um $n$-cubo fibrante $X$ temos funtores ${\aleph_{i}^{ \pm}}_{i} \operatorname{dos} n$-cubos fibrantes para os $(n-1)$-cubos fibrantes tais que

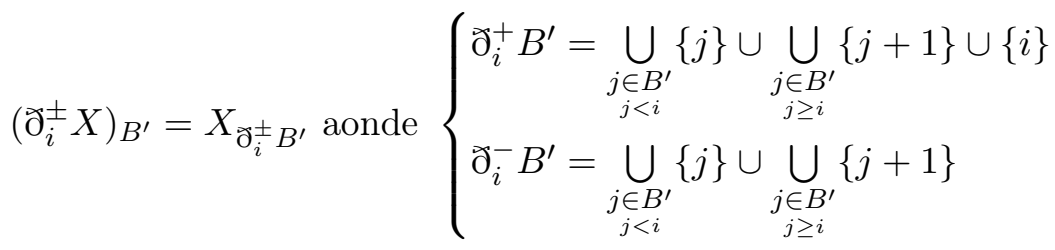

e tais que $\phi_{i}: \partial_{i}^{-} X \rightarrow \partial_{i}^{+} X$ é uma fibração de $(n-1)$-cubos fibrantes, ou equivalentemente um $(n-1)$-cubo de fibrações.

Portanto como todo $\phi_{n}:\left(\widetilde{\partial}_{n}^{+} X\right)_{B} \rightarrow\left(\widetilde{\partial}_{n}^{-} X\right)_{B}$, para $B \subseteq\langle n-1\rangle$, é uma fibração podemos aplicar o funtor $\mathscr{S}$ em cada uma dessas fibrações e obter um $(n-1)$-cubo de espaços simpliciais, denotado $\left(\mathscr{S}_{n} X\right)_{\bullet_{n}}$, tal que para todo $m \geq 0$ temos que $\left(\mathscr{S}_{n} X\right)_{m}$ é um $(n-1)$-cubo fibrante. Para cada $m \geq 0$ podemos então repetir esse processo na direção $n-1$ para obter um $(n-2)$-cubo de espaços simpliciais denotado $\left(\mathscr{S}_{n-1} \mathscr{S}_{n} X\right)_{\bullet, m}$, ou seja temos um $(n-2)$-cubo de espaços 2-simpliciais 


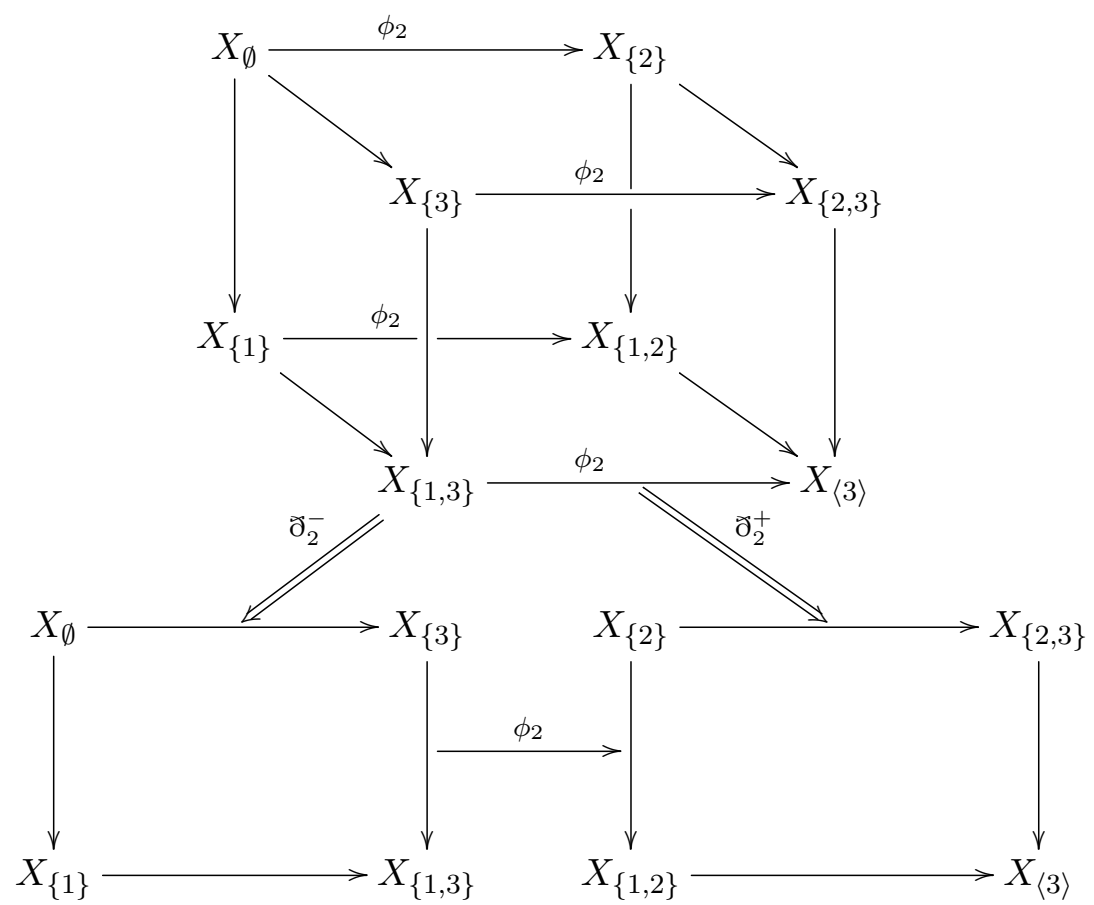

Figura 3.3: Funtores $\check{\partial}_{2}^{-}$e $\mathrm{\partial}_{2}^{+}$no caso $n=3$.

$\left(\mathscr{S}_{n-1} \mathscr{S}_{n} X\right)_{\left(\bullet_{n-1}, \bullet n\right)}$. Iterando esse processo $n$ vezes obtemos o espaço $n$-simplicial

$$
\operatorname{Simp}(X)=\left(\mathscr{S}_{1} \ldots \mathscr{S}_{n} X\right)_{\left(\bullet_{1} \ldots, \bullet_{n}\right)} \cdot
$$

Podemos tomar o grupo fundamental em cada espaço de $\operatorname{Simp}(X)$, e pela funtorialidade de $\pi_{1}$ obtemos um grupo $n$-simplicial

$$
\pi_{1} \circ \operatorname{Simp}(X)
$$

Além disso também existe um funtor da categoria dos grupos $n$-simpliciais a categoria dos précat $^{n}$-grupos denotado $\wedge$ com a seguinte descrição. Dado um grupo $n$-simplicial $G_{\left(\bullet_{1}, \ldots, \bullet_{n}\right)}$ definimos

$$
\begin{gathered}
\wedge\left(G_{\left(\bullet_{1}, \cdots \bullet_{n}\right)}\right)=G_{(1, \ldots, 1)}, \\
s_{i}=\partial_{0}^{i} \circ d_{0}^{i} \\
t_{i}=\partial_{1}^{i} \circ d_{0}^{i}
\end{gathered}
$$

aonde os axiomas $\left(\mathrm{C}^{n} \mathrm{G} 1\right)$, ( $\left.\mathrm{C}^{n} \mathrm{G} 2\right)$ e $\left(\mathrm{C}^{n} \mathrm{G} 4\right)-\left(\mathrm{C}^{n} \mathrm{G} 6\right)$ seguem diretamente da definição de objeto simplicial.

$$
\begin{aligned}
& -\partial_{1}^{i} \rightarrow \\
G_{1, \ldots, 1,1,1, \ldots, 1} & <d_{0}^{i}-G_{1, \ldots, 1,0,1, \ldots, 1} \\
& \longrightarrow \partial_{0}^{i} \rightarrow
\end{aligned}
$$

Dada essas preliminares podemos finalmente definir o funtor cat ${ }^{n}$-grupo fundamental.

Definição 3.2.1. O cat ${ }^{n}$-grupo fundamental $\Pi(X)$ de um n-cubo fibrante $X$ é dado por

$$
\Pi(X)=^{\wedge} \circ \pi_{1} \circ \operatorname{Simp}(X)
$$

No caso $n=0$ temos que $\Pi$ é simplesmente o grupo fundamental de um espaço topológico. O 
cat ${ }^{n}$-grupo fundamental de um $n$-cubo de fibrações $\bar{X}$ é simplesmente o cat ${ }^{n}$-grupo fundamental do $n$-cubo fibrante associado $X$.

$\mathrm{Como}^{\wedge}$ é um funtor da categoria dos grupos $n$-simpliciais à dos pré-cat ${ }^{n}$-grupos, já que para um grupo $n$-simplicial $G_{\left(\bullet_{1}, \cdots \bullet_{n}\right)}$ arbitrário não temos necessariamente que ${ }^{\wedge}\left(G_{\left(\bullet_{1}, \cdots \bullet_{n}\right)}\right)$ satisfaz $\left(\mathrm{C}^{n} \mathrm{G} 3\right)$, precisamos provar que esse axioma de fato é satisfeito para grupos $n$-simpliciais obtidos a partir de um $n$-cubo fibrante via $\pi_{1} \circ$ Simp. Os próximos lemas dessa seção nos darão esse resultado.

Lema 3.2.2. Seja $f: W \rightarrow Z$ uma fibração com fibra $F$, e seja $E_{\bullet}^{f}$ seu espaço simplicial associado. Então

i) o complexo de Moore do grupo simplicial $\pi_{1} E_{\bullet}^{f}$ é

$$
\cdots \rightarrow 1 \rightarrow 1 \rightarrow \pi_{1} F \stackrel{\bar{f}_{*}}{\rightarrow} \pi_{1} W
$$

ii) o grupo $\pi_{1} E_{2}^{f}$ é gerado por elementos degenerados, ou seja $\pi_{1} E_{2}^{f}=\operatorname{Im} \pi_{1} d_{0} \cup \operatorname{Im} \pi_{1} d_{1}$.

Demonstração: Temos que $\pi_{1}\left(E_{n}^{f}\right)=\left(\pi_{1}(F)\right)^{n} \rtimes \pi_{1}(W)$ [BHS11, prop. 2.6.1]. Os homomorfismos de bordo e degeneração de $\pi_{1} E_{n}^{f}$ são dados por

$$
\begin{aligned}
& \partial_{0}\left(\left(m_{1}, \ldots, m_{n}\right), w\right)=\left(\left(m_{2}, \ldots, m_{n}\right), w\right), \\
& \partial_{i}\left(\left(m_{1}, \ldots, m_{n}\right), w\right)=\left(\left(m_{1}, \ldots, m_{i} m_{i+1}, \ldots, m_{n}\right), w\right)(0<i<n), \\
& \left.\partial_{n}\left(\left(m_{1}, \ldots, m_{n}\right), w\right)=\left(\left(m_{1}, \ldots, m_{n-1}\right) \bar{f}_{*}\left(m_{n}\right) w\right)\right), \\
& d_{i}\left(\left(m_{1}, \ldots, m_{n}\right), w\right)=\left(\left(m_{1}, \ldots, m_{i}, 1, m_{i+1}, \ldots, m_{n}\right), w\right)(0 \leq i \leq n) .
\end{aligned}
$$

Logo $i$ ) e ii) seguem diretamente da descrição acima da estrutura simplicial de $\pi_{1} E_{\bullet}^{f}$ e da definição 3.1.11 do complexo de Moore de um grupo simplicial.

Lema 3.2.3. Seja $G_{\bullet}$ um grupo simplicial tal que $G_{2}$ é gerado por elementos degenerados. Então no complexo de Moore de $G_{\bullet}$ temos Im $\delta_{2}=\left[\operatorname{Ker} \partial_{1}, \operatorname{Ker} \partial_{0}\right]$.

Demonstração: Existem isomorfismos canônicos $G_{1} \cong(N G)_{1} \rtimes d_{0} G_{0}$ e $G_{2}=(N G)_{2} \rtimes$ $\left.d_{1}\left((N G)_{1}\right)\right) \rtimes\left(d_{0}\left((N G)_{1}\right) \rtimes d_{0} d_{0}\left(G_{0}\right)\right)$. Logo qualquer elemento em $G_{2}$ pode ser escrito de forma única $z=\bar{z} d_{1}(a) d_{0}\left(a^{\prime}\right) d_{0} d_{0}(u)$, com $\bar{z} \in(N G)_{2}, a, a^{\prime} \in(N G)_{1}, u \in G_{0}$. Seja $C$ o subgrupo normal de $(N G)_{2}$ gerado pelos comutadores $\left[d_{0}(x)^{-1} d_{1}(x), d_{1}(y)\right]$, com $x, y \in(N G)_{1}$. A imagem de $C$ por $\delta_{2}$, que é a restrição de $\partial_{0}$, é exatamente $\left[\operatorname{Ker} \partial_{1}\right.$, Ker $\left.\partial_{0}\right]$ pois todo elemento de Ker $\partial_{1}$ pode ser escrito como $\partial_{0} d_{0}\left(x^{-1}\right) x$, com $x \in(N G)_{1}$. Logo basta provar que $C=(N G)_{2}$, ou equivalentemente que em $G_{2} / C$ todo elemento pode ser escrito como $d_{1}(a) d_{0}\left(a^{\prime}\right) d_{0} d_{0}(u)$, com $a, a^{\prime} \in(N G)_{1}, u \in G_{0}$. Como $G_{2}$ é gerado por elementos degenerados basta mostrar que isso é verdade para os produtos de $d_{1}(a) d_{0}\left(a^{\prime}\right) d_{0} d_{0}(u)$ com $d_{0} d_{0}(v), d_{0}\left(b^{\prime}\right)$ e $d_{1}(b)$, para $v \in G_{0}$ e $b, b^{\prime} \in(N G)_{1}$.

Caso 1) O primeiro caso é imediato

$$
d_{1}(a) d_{0}\left(a^{\prime}\right) d_{0} d_{0}(u) d_{0} d_{0}(v)=d_{1}(a) d_{0}\left(a^{\prime}\right) d_{0} d_{0}(u v)
$$

Caso 2) No segundo temos

$$
\begin{aligned}
d_{1}(a) d_{0}\left(a^{\prime}\right) d_{0} d_{0}(u) d_{0}\left(b^{\prime}\right) & =d_{1}(a) d_{0}\left(a^{\prime}\right) d_{0}\left(d_{0}(u) b^{\prime} d_{0}(u)^{-1}\right) d_{0} d_{0}(u) \\
& =d_{1}(a) d_{0}\left(a^{\prime} d_{0}(u) b^{\prime} d_{0}(u)^{-1}\right) d_{0} d_{0}(u)
\end{aligned}
$$


Caso 3) No terceiro caso precisamos usar que em $G_{2} / C$ temos a identidade $d_{0}(x) d_{1}(y) \cong d_{1}\left(x y x^{-1}\right) d_{0}(x)$

$$
\begin{aligned}
d_{1}(a) d_{0}\left(a^{\prime}\right) d_{0} d_{0}(u) d_{1}(b) & \cong d_{1}(a) d_{0}\left(a^{\prime}\right) d_{1}\left(d_{0}(u) b d_{0}(u)^{-1}\right) d_{0} d_{0}(u) \\
& \cong d_{1}(a) d_{1}\left(a^{\prime} d_{0}(u) b d_{0}(u)^{-1} a^{\prime-1}\right) d_{0}\left(a^{\prime}\right) d_{0} d_{0}(u) \\
& =d_{1}\left(a a^{\prime} d_{0}(u) b d_{0}(u)^{-1} a^{-1}\right) d_{0}\left(a^{\prime}\right) d_{0} d_{0}(u) .
\end{aligned}
$$

Logo $C=(N G)_{2}$ e o lema está provado

Usando estes dois lemas podemos provar o que queríamos.

Teorema 3.2.4. Para todo $X$ n-cubo fibrante, $\Pi(X)$ é um cat $^{n}$-grupo.

Demonstração: Usando o lema 3.2.2.ii temos que o lema3.2.3 nos dá que para todo $i \in\langle n\rangle$ temos que [Ker $s_{i}$, Ker $t_{i}$ ] em $\Pi(X)$ é a imagem de $\delta_{2}$ do complexo de Moore do grupo simplicial $\left(\pi_{1} \circ \operatorname{Simp}(X)\right)_{1, \ldots, 1, \bullet, 1, \ldots, 1}$ com o índice variando na coordenada $i$. Como pelo lema 3.2.2.i temos que o grupo de dimensão 2 nesse complexo de Moore é trivial concluímos que $\Pi(X)$ satisfaz $\left(\mathrm{C}^{n} \mathrm{G} 3\right)$.

\subsection{Equivalência de $n$-cubos de Eilenberg-MacLane e cat $^{n}$-grupos}

Seguindo as ideias encontradas em [Lod82] descreveremos agora o funtor espaço de classificação que associa um $(n+1)$-tipo homotópico a cada cat ${ }^{n}$-grupo. Também precisaremos construir um funtor $n$-cubo de fibrações fundamental, que mostraremos que, junto com $\Pi$, forma uma equivalência entre as categorias dos cat $^{n}$-grupos e a dos $n$-cubos de Eilenberg-MacLane.

Lembremos da seção 2.2 que um cat $^{n}$-grupo contém a informação de uma $n$-categoria interna a categoria dos grupos. Isso significa que podemos criar, a partir de um cat ${ }^{n}$-grupo $G, n$ estruturas de categoria interna aos grupos independentes, ou seja que para cada $i \in\langle n\rangle$ temos a categoria interna aos grupos

$$
G \underset{t_{i}}{\stackrel{s_{i}}{\rightleftarrows}} \operatorname{Im} s_{i}
$$

Aplicando o funtor nervo $\mathcal{N}$ da definição 3.1.9 nessa estrutura categorial obtemos um cat ${ }^{(n-1)}$ grupo simplicial $\mathcal{N}_{i} G$. Iterando esse processo obtemos um grupo $n$-simplicial $\mathcal{N}_{1} \ldots \mathcal{N}_{n}(G)$. Como vimos na primeira seção 3.1 podemos tomar o nervo desse grupo $n$-simplicial, obtendo um conjunto $(n+1)$-simplicial que denotaremos por $\underline{\operatorname{Simp}}(G)$.

Definição 3.3.1. O espaço de classificação $B(G)$ do cat ${ }^{n}$-grupo $G$ é dado por

$$
B(G)=|D \circ \underline{\operatorname{Simp}}(G)|=\left|D \circ \mathcal{N} \circ \mathcal{N}_{1} \ldots \mathcal{N}_{n}(G)\right|
$$

O seguinte lema nos mostra que o funtor $B$ transforma sequências exatas curtas em fibrações.

Lema 3.3.2. Se

$$
1 \rightarrow G^{\prime} \stackrel{\alpha}{\rightarrow} G \stackrel{\beta}{\rightarrow} G^{\prime \prime} \rightarrow 1
$$

for uma sequencia exata de cat ${ }^{n}$-grupos então

$$
B\left(G^{\prime}\right) \stackrel{\alpha}{\rightarrow} B(G) \stackrel{\beta}{\rightarrow} B\left(G^{\prime \prime}\right)
$$

é uma fibração. 
Demonstração: Provaremos que $\beta$ induz uma fibração de Kan nos conjuntos simpliciais

$$
\beta_{\bullet}:(D \circ \underline{\operatorname{Simp}}(G)) \bullet \rightarrow\left(D \circ \underline{\operatorname{Simp}}\left(G^{\prime \prime}\right)\right) \bullet
$$

com as fibras dadas pelas inclusões induzidas por $\alpha$. O lema segue então do teorema 3.1.14.

Por funtorialidade temos que $\beta$ induz um morfismo de conjuntos simpliciais, que é fácil ver que será sobrejetor. Para todo chifre $\left(g_{0}, \ldots, g_{k-1}, g_{k}, \ldots, g_{m}\right) \in(D \circ \underline{\operatorname{Simp}}(G))_{m-1}^{m}$ tal que exista $g^{\prime \prime} \in\left(D \circ \operatorname{Simp}\left(G^{\prime \prime}\right)\right)_{m} \operatorname{com} \partial_{i}\left(g^{\prime \prime}\right)=\beta_{m-1}\left(g_{i}\right)$ podemos, já que $\beta$ • é sobrejetora, simplesmente tomar como completamento do chifre $g \in \beta^{-1}\left(g^{\prime \prime}\right)$ qualquer. Disso claramente segue que a inclusão das fibras é induzida por $\alpha$.

Será útil descrever a imagem por $B$ de dois tipos especiais de cat ${ }^{n}$-grupos.

Lema 3.3.3. Se $G$ é um cat ${ }^{n}$-grupo cujos endomorfismos $s_{i}$ e $t_{i}$ são todos a identidade então $B(G)=K(G, 1)$.

Demonstração: Como para todo $i \in\langle n\rangle$ temos $s_{i}=\mathbb{1}_{G}=t_{i}$ temos que $G \times \times_{\operatorname{Im~} s_{i}} G \cong G$, logo o grupo $n$-simplicial $\mathcal{N}_{1} \ldots \mathcal{N}_{n} G$ é dado inteiramente pelo grupo $G$ e por identidades. Como o complexo de Moore da diagonal de $\mathcal{N}_{1} \ldots \mathcal{N}_{n} G$ é portanto trivial em todas as dimensões exceto a 0 , aonde ele é $G$, temos pelo teorema 3.1.12 que $\pi_{k}(B(G))=0$ para $k>1$, e que $\pi_{1}(B(G))=G$.

Lema 3.3.4. Seja $G$ um cat ${ }^{n}$-grupo tal que para algum subconjunto não vazio $A$ de $\langle n\rangle$ temos que $t_{i}\left\lceil_{\text {Ker } s_{i}}:\right.$ Ker $s_{i} \rightarrow \operatorname{Im} s_{i}$ é um isomorfismo para todo $i \in A$, e $s_{i}=t_{i}=\mathbb{1}$ para $i \notin A$. Então $B(G)$ é contrátil.

Demonstração: Por $s_{i}=t_{i}=\mathbb{1}$ para $i \notin A$ temos que $B(G)=B\left(G^{\prime}\right)$ aonde $G^{\prime}$ é o cat $\left.{ }^{|A|}\right|_{\text {-grupo }}$ associado aos endomorfismos com índices em $A$. Temos que para cada $i \in A$ a estrutura categorial associada a $i$ gera um cat ${ }^{(n-1)}$-grupo simplicial cujo complexo de Moore tem homologia trivial em todas as dimensões, o que implica que os grupos de homotopia de $B\left(G^{\prime}\right)$ são todos triviais

Para a construção dos $n$-cubos fibrantes associados a um cat $^{n}$-grupo precisamos definir funtores $\Gamma_{i}^{j}$, para $j=-1,0,1$ e $i \in\langle n\rangle$, da categoria de cat $^{n}$-grupos para si mesma como sendo

$$
\Gamma_{i}^{-1}(G)=\operatorname{Ker} s_{i}
$$

com $s_{i}$ e $t_{i}$ a identidade e as outras $s_{k}$ e $t_{k}$ dadas pelas devidas restrições;

$$
\Gamma_{i}^{0}(G)=\operatorname{Ker} s_{i} \rtimes G
$$

Aonde a ação de $G$ em Ker $s_{i}$ é dada por conjugação, com $s_{i}(k, g)=(1, g)$ e $t_{i}(k, g)=(1, k g)$, e com os outros $s_{k}$ e $t_{k}$ dados pela restrição em cada componente;

$$
\Gamma_{i}^{1}(G)=G
$$

Também temos as transformações

$$
\begin{gathered}
\Gamma_{i}^{-1} \stackrel{\alpha_{i}}{\rightarrow} \Gamma_{i}^{0} \stackrel{\beta_{i}}{\longrightarrow} \Gamma_{i}^{1} \\
\alpha_{i}(k)=\left(1, k^{-1} t_{i}(k)\right) \\
\beta_{i}(k, g)=k t_{i}(g)
\end{gathered}
$$


de forma que

$$
1 \rightarrow \Gamma_{i}^{-1}(G) \stackrel{\alpha_{i}}{\longrightarrow} \Gamma_{i}^{0}(G) \stackrel{\beta_{i}}{\longrightarrow} \Gamma_{i}^{1}(G) \rightarrow 1
$$

sempre é exato. Para $A, B \subseteq\langle n\rangle$ disjuntos denotamos $\Gamma^{A, B}=\Gamma_{1}^{\varepsilon_{1}} \circ \cdots \circ \Gamma_{n}^{\varepsilon_{n}}$, com $\varepsilon_{i}=1$ se $i \in B$, $\varepsilon_{i}=-1$ se $i \in A$ e $\varepsilon_{i}=0$ se $i \in\langle n\rangle \backslash(A \cup B)$.

Devido ao lema 3.3.2 o funtor descrito a seguir está bem definido.

Definição 3.3.5. O funtor $n$-cubo de fibrações de classificação $\mathcal{B}$ é definido por

$$
\mathcal{B}(G)_{A, B}=B \circ \Gamma^{A, B}(G)
$$

e com as fibrações induzidas pelos $\alpha_{i}$ e $\beta_{i}$.

A equivalência entre as categorias de $n$-cubos de Eilenberg-MacLane e cat ${ }^{n}$-grupos será consequência dos próximos teoremas.

Teorema 3.3.6. Os espaços $\mathcal{B}(G)_{A, \emptyset}$ são espaços de Eilenberg-MacLane do tipo $K(\pi, 1)$.

Demonstração: Defina $\bar{\Gamma}_{i}^{-1}=\Gamma_{i}^{-1}, \bar{\Gamma}_{i}^{0}(G)=\operatorname{Im} s_{i}$ e $\bar{\Gamma}^{A}=\bar{\Gamma}_{1}^{\varepsilon_{1}} \circ \cdots \circ \bar{\Gamma}_{n}^{\varepsilon_{n}} \operatorname{com} \varepsilon_{i}=-1$ se $i \in A$ e $\varepsilon_{i}=0$ se $i \in\langle n\rangle \backslash A$.

Temos então sequencias exatas curtas

$$
1 \rightarrow \underset{j \notin A}{\rtimes} \underset{i \in\langle n\rangle}{\cap} \operatorname{Ker} s_{i} \rightarrow \Gamma^{A, \emptyset}(G) \stackrel{\theta}{\rightarrow} \bar{\Gamma}^{A}(G) \rightarrow 1
$$

com a função $\theta$ definida da seguinte forma. Temos uma coordenada de $\Gamma^{A, \emptyset}(G)$ para cada subconjunto $B \subseteq\langle n\rangle \backslash A$, sendo que essa coordenada é dada por $\cap$ Ker $s_{i}$. Dada uma ordem total para os subconjuntos de $\langle n\rangle \backslash A$ definimos $\theta\left(\left(k_{B}\right)_{B \subseteq\langle n\rangle \backslash A}\right)=\prod_{B \subseteq\langle n\rangle \backslash A}\left(\underset{i \in\langle n\rangle \backslash A \cup B}{\circ} s_{i}\right) \circ\left(\underset{i \in B}{\circ} t_{i}\right)\left(k_{B}\right)$.

Pelo lema 3.3.4 temos que $B \underset{j \notin A}{\rtimes} \underset{i \in\langle n\rangle}{\cap}$ Ker $\left.s_{i}\right)$ é contrátil, e portanto pelo lema 3.3.2 temos que $B\left(\Gamma^{A, \emptyset}(G)\right) \stackrel{B(\theta)}{\longrightarrow} B\left(\bar{\Gamma}^{A}(G)\right)$ é uma equivalência homotópica. Note que ambos $\bar{\Gamma}_{i}^{-1}$ e $\bar{\Gamma}_{i}^{0}$ levam $G$ a um cat ${ }^{n}$-grupo tal que $s_{i}$ e $t_{i}$ são identidades, logo todos os endomorfismos $s_{i}$ e $t_{i}$ de $\bar{\Gamma}^{A}(G)$ são a identidade e portanto, pelo lema 3.3.3, $B\left(\bar{\Gamma}^{A}(G)\right)=K\left(\bar{\Gamma}^{A}(G), 1\right)$

Teorema 3.3.7. A composição $\Pi \circ \mathcal{B}$ é a identidade.

Demonstração: Mostraremos que para todo cat ${ }^{n}$-grupo $G$ e $i \in\langle n\rangle$ o funtor $\Pi \circ \mathcal{B}$ preserva a estrutura de cat ${ }^{1}$-grupo na direção $i$. Defina $G_{i}^{\prime}$ como o cat $^{n}$-grupo cujo grupo subjacente e todos os endomorfismos $s_{j}$ e $t_{j}$ para $j \neq i$ são iguais aos de $G$ e com $s_{i}$ e $t_{i}$ agora dados pela identidade. Consideremos agora o quadrado comutativo

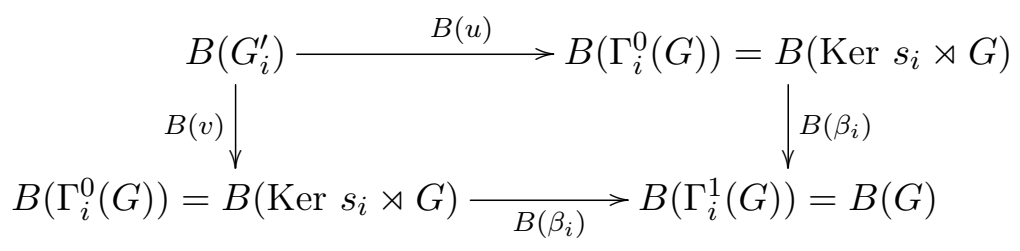

aonde $u(g)=\left(1, s_{i}(g) g^{-1} t_{i}(g)\right)$ e $v(g)=\left(1, s_{i}(g)\right)$ ( $u$ é um homomorfismo pelo axioma $\left(\mathrm{C}^{n} \mathrm{G} 3\right)$ ). Pelo lema 3.3.2 ambas as fibras das aplicações verticais são $B\left(\Gamma_{i}^{-1}(G)\right)=B\left(\operatorname{Ker} s_{i}\right)$, e além disso podemos ver que $B(u)$ induz a identidade entre as fibras. Portanto o diagrama acima é um 
pullback e $B\left(G_{i}^{\prime}\right)=B\left(\Gamma_{i}^{0}(G)\right) \times_{B\left(\Gamma_{i}^{1}(G)\right)} B\left(\Gamma_{i}^{0}(G)\right)$. Repetindo esse argumento para cada $i \in\langle n\rangle$ vemos que $(\operatorname{Simp} \circ \mathcal{B}(G))_{1 \ldots 1}$ é simplesmente $B\left(G^{\prime}\right)$, para $G^{\prime}$ o grupo subjacente de $G$, portanto $\pi_{1}\left((\operatorname{Simp} \circ \mathcal{B}(G))_{1 \ldots 1}\right)=G^{\prime}$.

Também temos que $(\operatorname{Simp} \circ \mathcal{B}(G))_{1 \ldots 101 \ldots 1}$ com o zero na posição $i$ é, pelo mesmo argumento, simplesmente $B\left(\operatorname{Ker} s_{i} \rtimes G\right)$ com a estrutura de cat ${ }^{1}$-grupo dada por $s(m, g)=(1, g)$ e $t(m, g)=$ $(1, g m)$. Temos que o grupo simplicial $\mathcal{N}_{1}\left(\right.$ Ker $\left.s_{i} \rtimes G\right)$ tem como morfismos entre a dimensão $1 \mathrm{e}$ 0 dados por $s, t:$ Ker $s_{i} \rtimes G \rightarrow \operatorname{Im} s \operatorname{com} \operatorname{Im} s \cong G$, Ker $s \cong$ Ker $s_{i}$ e a restrição de $t$ sendo a inclusão do Ker $s_{i}$ em $G$, $\operatorname{logo} \pi_{1}\left(B\left(\operatorname{Ker} s_{i} \rtimes G\right)\right)=H_{0}\left(\mathcal{N}_{1}\left(G \ltimes \operatorname{Ker} s_{i}\right)\right) \cong \operatorname{Im} s_{i}$. Além disso temos que $v$ no diagrama acima induz $s_{i}$ e o o homomorfismo $u$ induz $t_{i}$, já que $s_{i}(g) g^{-1} \in$ Ker $s_{i}$ para todo $g \in G$

Teorema 3.3.8. Para todo $n$-cubo de fibrações $\bar{X}$ existe um morfismo de $n$-cubos de fibração $\bar{X} \rightarrow$ $\mathcal{B} \circ \Pi(\bar{X})$, bem definido a menos de homotopia, de forma que para todo par de subconjuntos disjuntos $A, B \subseteq\langle n\rangle$ temos que $\pi_{1}\left(\bar{X}_{A, B}\right) \rightarrow \pi_{1}\left(\mathcal{B} \circ \Pi(\bar{X})_{A, B}\right)$ é o homomorfismo identidade.

Demonstração: Começaremos construindo um morfismo $\bar{X}_{\emptyset,\langle n\rangle} \rightarrow B \circ \Pi(\bar{X})$, bem definido a menos de homotopia, que induz um isomorfismo em $\pi_{1}$. A resolução geométrica de $\operatorname{Simp}(\bar{X})$ tem a propriedade de ser homotopicamente equivalente ao espaço $\bar{X}_{\emptyset,\langle n\rangle}$. Como para todo espaço $Z$ existe a menos de homotopia um morfismo $Z \rightarrow B \circ \pi_{1}(Z)=\left|D \circ \mathcal{N} \circ \pi_{1}(Z)\right|$ que induz um isomorfismo em $\pi_{1}$, temos que existe a menos de homotopia um morfismo de espaços $n$-simpliciais $\operatorname{Simp}(\bar{X})_{\left(\bullet_{1}, \ldots, \bullet_{n}\right)} \rightarrow B\left(\left(\pi_{1} \circ \operatorname{Simp}(\bar{X})\right)_{\left(\bullet_{1}, \ldots, \bullet_{n}\right)}\right)$ que induz um isomorfismo em $\pi_{1}$ em todo índice. Além disso como $\mathcal{N}_{1} \ldots \mathcal{N}_{n} \circ^{\wedge}=\mathbb{1}$ temos que $\pi_{1} \circ \operatorname{Simp}(\bar{X})=\mathcal{N}_{1} \ldots \mathcal{N}_{n} \Pi(\bar{X})$. Tomando a realização geométrica obtemos o morfismo desejado.

$\bar{X}_{\emptyset,\langle n\rangle} \cong\left|(\operatorname{Simp}(\bar{X}))_{\left(\bullet_{1}, \ldots, \bullet_{n}\right)}\right| \rightarrow\left|B\left(\left(\pi_{1} \circ \operatorname{Simp}(\bar{X})\right)_{\left(\bullet_{1}, \ldots, \bullet_{n}\right)}\right)\right|=\left|D \circ \mathcal{N} \circ \mathcal{N}_{1} \ldots \mathcal{N}_{n} \circ \Pi(\bar{X})\right|=B \circ \Pi(\bar{X})$

Agora para construir $\bar{X} \rightarrow \mathcal{B} \circ \Pi(\bar{X})$ precisamos primeiro definir uma família de funtores $\gamma_{i}^{j}$, para $j=-1,0,1$ e $i \in\langle n\rangle$, da categoria dos $n$-cubos de fibração para si mesmos como sendo

$$
\begin{aligned}
& \gamma_{i}^{-1}(\bar{X})_{A, B}= \begin{cases}* & \text { se } i \in A \\
\bar{X}_{A \cup\{i\}, B \backslash\{i\}} & \text { se } i \notin A\end{cases} \\
& \gamma_{i}^{0}(\bar{X})_{A, B}= \begin{cases}\bar{X}_{A, B} & \text { se } i \in A \\
\bar{X}_{A, B} \times_{\bar{X}_{A, B \cup\{i\}}} \bar{X}_{A, B} & \text { se } i \notin A \text { e } i \notin B \\
X_{A, B \backslash\{i\}} & \text { se } i \in B\end{cases} \\
& \gamma_{i}^{1}(\bar{X})_{A, B}=\bar{X}_{A, B}
\end{aligned}
$$

Para todo $A, B \subseteq\langle n\rangle$ definimos $\gamma^{A, B}=\gamma_{1}^{\varepsilon_{1}} \circ \cdots \circ \gamma_{n}^{\varepsilon_{n}}$, com $\varepsilon_{i}=1$ se $i \in B, \varepsilon_{i}=-1$ se $i \in A$ e $\varepsilon_{i}=0$ se $i \in\langle n\rangle \backslash(A \cup B)$. Note que $\Pi \circ \gamma^{A, B}=\Gamma^{A, B} \circ \Pi$. A definição implica que $\gamma^{A, B}(\bar{X})_{\emptyset,\langle n\rangle}=\bar{X}_{A, B}$, logo temos os morfismos

$$
\bar{X}_{A, B}=\gamma^{A, B}(\bar{X})_{\emptyset,\langle n\rangle} \rightarrow B\left(\Pi \circ \gamma^{A, B}(\bar{X})\right)=B\left(\Gamma^{A, B} \circ \Pi(\bar{X})\right)=\mathcal{B} \circ \Pi(\bar{X})_{A, B}
$$

que formam o morfismo que queriamos 
Corolario 3.3.9. Os funtores $\Pi$ e $\mathcal{B}$ formam uma equivalência da categoria homotópica de n-cubos de Eilenberg-MacLane com a categoria de cat ${ }^{n}$-grupos.

Demonstração: O teorema segue dos teoremas 3.3.6,3.3.7 e 3.3.8, e observando que nos casos dos $n$-cubos de Eilenberg-MacLane as sequencias exatas das fibrações implicam que o morfismo dado no teorema 3.3.8 é uma equivalência.

Note que o caso $n=0$ é simplesmente o resultado clássico que a categoria de espaços de Eilenberg-MacLane do tipo $K(\pi, 1)$ é equivalente à categoria dos grupos.

\subsection{Grupos de homotopia do espaço de classificação de um cat $^{n}$-grupo}

Juntando os resultados desse capítulo com os do anterior vemos que todo cat ${ }^{n}$-grupo $G$ está associado a um $(n+1)$-tipo homotópico dado por $B(G)$. Ainda seguindo ideias encontradas em [Lod82] investigaremos nessa seção como calcular os grupos de homotopia $\pi_{i} \circ B(G)$ e daremos uma definição de quasi-isomorfismo de cat $^{n}{ }^{n}$-grupos que induz uma equivalência homotópica fraca entre os espaços de classificação.

Para isso precisaremos generalizar certas construções usuais da álgebra homológica de forma que ela possa ser usada para lidar com grupos que não sejam necessariamente abelianos. Sugerimos como referencia para a teoria de álgebra homológica no contexto original de grupos abelianos o livro introdutório de Weibel [Wei95].

Definição 3.4.1. Um complexo de grupos (não necessariamente abelianos) de tamanho n, denotado $\left(A_{\bullet}, d_{\bullet}\right)$, é uma sequencia de homomorfismos de grupos

$$
\cdots \rightarrow A_{n} \stackrel{d_{n}}{\longrightarrow} A_{n-1} \stackrel{d_{n-1}}{\longrightarrow} \cdots \stackrel{d_{2}}{\longrightarrow} A_{1} \stackrel{d_{1}}{\longrightarrow} A_{0} \rightarrow \cdots
$$

tal que $A_{i}=1$ para $i<0$ e $i>n$ e $\operatorname{Im} d_{i+1}$ é um subgrupo normal de Ker $d_{i}$. Note que os grupos de homologia $H_{i}\left(A_{\bullet}\right)=\frac{\operatorname{Ker} d_{i}}{\operatorname{Im} d_{i+1}}$ estão bem definidos.

Definição 3.4.2. Um morfismo de complexos de grupos $f$ de $\left(A_{\bullet}, d_{\bullet}\right)$ em $\left(B_{\bullet}, d_{\bullet}^{\prime}\right)$ é uma sequencia de homomorfismos de grupos $f_{i}: A_{i} \rightarrow B_{i}$ que comutam com os homomorfismos dos complexos $\left(A_{\bullet}, d_{\bullet}\right)$ e $\left(B_{\bullet}, d_{\bullet}^{\prime}\right)$. Se esse morfismo induz isomorfismos nas homologias dizemos que $f$ é um quasiisomorfismo.

Diferentemente do caso em que todos os grupos são abelianos nem sempre existe um cone para esses morfismos de complexos de grupos, porém um cone existe se assumirmos alguma estrutura extra.

Lema 3.4.3. Seja $f$ um morfismo de complexos de grupos de tamanho $n$ tal que para $0 \leq i \leq n$ existe uma ação de $B_{i}$ em $A_{i}$ fazendo com que $f_{i}: A_{i} \rightarrow B_{i}$ seja um módulo cruzado e tal que os pares $\left(d_{i}, d_{i}^{\prime}\right)$ formem morfismos de módulos cruzados. Se definirmos $C_{i}=A_{i-1} \rtimes B_{i}$ com a ação de $B_{i}$ em $A_{i-1}$ dada pela ação de sua imagem por $d_{i}^{\prime}$, e $\delta_{i+1}: C_{i+1} \rightarrow C_{i}$ dada por

$$
\delta_{i+1}(a, b)=\left(d_{i}(a)^{-1}, f_{i}(a) d_{i+1}^{\prime}(b)\right)
$$

então $\left(C_{\bullet}, \delta_{\bullet}\right)$ é um complexo de grupos de tamanho $n+1$. 
Demonstração: Claramente $\delta_{i+1}(1, b) \delta_{i+1}\left(1, b^{\prime}\right)=\delta_{i+1}\left(1, b b^{\prime}\right)$. Também temos

$$
\begin{aligned}
& \delta_{i+1}(a, 1) \delta_{i+1}\left(a^{\prime}, 1\right)=\left(d_{i}(a)^{-1}, f_{i}(a)\right)\left(d_{i}\left(a^{\prime}\right)^{-1}, f_{i}\left(a^{\prime}\right)\right)
\end{aligned}
$$

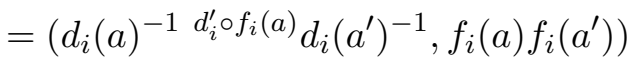

$$
\begin{aligned}
& =\left(d_{i}(a)^{-1 f_{i-1} \circ d_{i}(a)} d_{i}\left(a^{\prime}\right)^{-1}, f_{i}\left(a a^{\prime}\right)\right) \\
& =\left(d_{i}(a)^{-1} d_{i}(a) d_{i}\left(a^{\prime}\right)^{-1} d_{i}(a)^{-1}, f_{i}\left(a a^{\prime}\right)\right) \\
& =\left(d_{i}\left(a a^{\prime}\right)^{-1}, f_{i}\left(a a^{\prime}\right)\right) \\
& =\delta_{i+1}\left(a a^{\prime}, 1\right)
\end{aligned}
$$

e

$$
\begin{aligned}
\delta_{i+1}(1, b) \delta_{i+1}(a, 1) & =\left(1, d_{i+1}^{\prime}(b)\right)\left(d_{i}(a)^{-1}, f_{i}(a)\right) \\
& =\left(d_{i}^{\prime} \circ d_{i+1}^{\prime}(b)\right. \\
i & \left.(a)^{-1}, d_{i+1}^{\prime}(b) f_{i}(a)\right) \\
& =\left(d_{i}\left({ }^{\prime}{ }_{i+1}^{\prime}(b) a\right)^{-1}, f_{i}\left(d^{\prime}{ }^{\prime}(b) a\right) d_{i+1}^{\prime}(b)\right) \\
& =\delta_{i+1}\left({ }^{b} a, b\right)
\end{aligned}
$$

portanto os $\delta_{i+1}$ são homomorfismos de grupos.

Como $\left(a^{\prime}, b^{\prime}\right) \in \operatorname{Ker} \delta_{i}$ se, e somente se, $d_{i}^{\prime}\left(b^{\prime}\right)=f_{i+1}\left(a^{\prime}\right)^{-1}$, e como Im $d_{i+1}^{\prime}$ é normal no kernel de $d_{i}^{\prime}$ existe $b^{\prime \prime} \in B_{i+1}$ tal que $b^{\prime} d_{i+1}^{\prime}(b) b^{\prime-1}=d_{i+1}^{\prime}\left(b^{\prime \prime}\right)$ temos que

$$
\begin{aligned}
\left(a^{\prime}, b^{\prime}\right) \delta_{i+1}(a, b)\left({ }^{\prime}\left(b^{\prime}\right)^{-1} a^{\prime-1}, b^{\prime-1}\right) & =\left(a^{\prime}, b^{\prime}\right)\left(d_{i}(a)^{-1}, f_{i}(a) d_{i+1}^{\prime}(b)\right)\left(d_{i}^{\prime}\left(b^{\prime}\right)^{-1} a^{\prime-1}, b^{-1}\right) \\
& =\left(a^{\prime} d_{i}^{\prime}\left(b^{\prime}\right) d_{i}(a)^{-1} d_{i}^{\prime}\left(b^{\prime} f_{i}(a) d_{i+1}^{\prime}(b) b^{\prime-1}\right) a^{\prime-1}, b^{\prime} f_{i}(a) d_{i+1}^{\prime}(b) b^{\prime-1}\right) \\
& =\left(a^{\prime} f_{i-1}\left(a^{\prime}\right)^{-1} d_{i}(a)^{-1} f_{i-1}\left(a^{\prime}\right)^{-1} d_{i}^{\prime \circ} f_{i}(a) f_{i-1}\left(a^{\prime}\right) a^{\prime-1}, b^{\prime} f_{i}(a) b^{\prime-1} b^{\prime} d_{i+1}^{\prime}(b)^{-1} b^{\prime}\right) \\
& =\left(a^{\prime} a^{\prime-1} d_{i}(a)^{-1} a^{\prime} a^{\prime-1} d_{i}(a) a^{\prime} a^{\prime-1} a^{\prime-1} d_{i}(a)^{-1} a^{\prime}, f_{i}\left(b^{\prime} a\right) d_{i+1}^{\prime}\left(b^{\prime \prime}\right)\right) \\
& =\left(a^{\prime-1} d_{i}(a)^{-1} a^{\prime}, f_{i}\left(b^{\prime} a\right) d_{i+1}^{\prime}\left(b^{\prime \prime}\right)\right) \\
& =\left({ }^{\prime}\left(b^{\prime}\right) d_{i}(a)^{-1}, f_{i}\left(b^{\prime} a\right) d_{i+1}^{\prime}\left(b^{\prime \prime}\right)\right) \\
& =\left(d_{i}\left(b^{\prime} a\right)^{-1}, f_{i}\left(b^{\prime} a\right) d_{i+1}^{\prime}\left(b^{\prime \prime}\right)\right) \\
& =\delta_{i+1}\left({ }^{b^{\prime}} a, b^{\prime \prime}\right)
\end{aligned}
$$

temos que a imagem de $\delta_{i+1}$ é normal no kernel de $\delta_{i}$.

Definição 3.4.4. Seja $f$ um morfismo de complexos de grupos como o do enunciado do lema 3.4.3. Então o complexo de grupo $\left(C_{\bullet}, \delta_{\bullet}\right)$ de tamanho $n+1$ descrito no lema é o cone de $f$.

O resultado abaixo justifica a nomenclatura dada na definição anterior, já que ele diz que $\left(C_{\bullet}, \delta_{\bullet}\right)$ se comporta como um cone de um morfismo de complexos de grupos abelianos se comporta.

Teorema 3.4.5. Seja $f$ um morfismo de complexos de grupos como o do enunciado do lema 3.4.3 $e\left(C_{\bullet}, \delta_{\bullet}\right)$ o cone de $f$. Então existe uma sequencia exata longa

$$
\cdots \rightarrow H_{i}\left(A_{\bullet}\right) \rightarrow H_{i}\left(B_{\bullet}\right) \rightarrow H_{i}\left(C_{\bullet}\right) \rightarrow H_{i-1}\left(A_{\bullet}\right) \rightarrow \cdots
$$

Demonstração: O homomorfismo $H_{i}\left(B_{\bullet}\right) \rightarrow H_{i}\left(C_{\bullet}\right)$ é por $b \mapsto(b, 1)$. O homomorfismo $H_{i}\left(C_{\bullet}\right) \rightarrow H_{i-1}\left(A_{\bullet}\right)$ é dado pela projeção $(b, a) \mapsto a^{-1}$, que não é um homomorfismo, mas sua 
restrição ao kernel dos $\delta$ é. Que os homomorfismos entre as homologias está bem definido segue da comutatividade do diagrama resultante. Exatidão segue por busca por diagrama ${ }^{1}$.

Finalmente, podemos construir o complexo de grupos associado a um cat ${ }^{n}$-grupo cujos grupos de homologia serão os grupos de homotopia do $n$-tipo homotópico associado a ele.

Definição 3.4.6. O complexo de grupos $C \cdot(G)$ associado a um cat ${ }^{n}$-grupo $G$ é definido recursivamente da seguinte forma. Considere $G$ um complexo de cat ${ }^{n}$-grupos de tamanho 0. Para cada $1 \leq i \leq n$ temos um módulo cruzado de cat ${ }^{n-1}$-grupos associado a $G$, que podemos pensar como um morfismo de complexos de cat ${ }^{n-1}$-grupos que satisfaz as hipóteses do lema 3.4 .3 e o seu cone é um complexo de cat $^{n-1}$-grupos de tamanho 1 . Repetindo esse procedimento $n$ vezes obtemos um complexo de grupos de tamanho $n$ que denotaremos $\left(C_{\bullet}(G), \delta_{\bullet}\right)$. Um homomorfismo de cat ${ }^{n}$-grupos é um quasi-isomorfismo se o homomorfismo de complexos de grupos induzido é um quasi-isomorfismo.

Com essa definição vemos que existem grupos de homologia associados a todo cat ${ }^{n}$-grupo dados pelos grupos de homologia de $C_{\bullet}(G)$. Mostraremos agora que esses grupos coincidem com os grupos de homotopia de $B(G)$.

Teorema 3.4.7. Para todo cat ${ }^{n}$-grupo $G$ temos que $\pi_{i}(B(G))=H_{i-1}\left(C_{\bullet}(G)\right)$.

Demonstração: Para o caso $n=0$ isso é óbvio. Assuma que isso é verdade para cat $^{n-1}$ grupos. Denotemos por $C_{\bullet}^{N}(G)$ o complexo de Moore de $D \circ \mathcal{N}_{1} \ldots \mathcal{N}_{n}(G)$. Provaremos que existe um morfismo de complexos $\varepsilon(G): C_{\bullet}^{M}(G) \rightarrow C_{\bullet}(G)$ que é um quasi-isomorfismo.

Seja $U_{\bullet}=V_{\bullet} \rtimes W_{\bullet}$ um cat ${ }^{1}$-grupo simplicial. O complexo de Moore de $U_{\bullet}$ é um complexo de cat $^{1}$-grupos e portanto podemos construir o seu cone como na definição anterior.

$$
\cdots \rightarrow V_{2}^{\prime} \rtimes W_{3}^{\prime} \rightarrow V_{1}^{\prime} \rtimes W_{2}^{\prime} \rightarrow V_{0} \rtimes W_{1}^{\prime} \rightarrow W_{0}
$$

Por outro lado aplicando o funtor nervo $\mathcal{N}$ nas estruturas categoriais de cada cat ${ }^{1}$-grupo em $U$ • obtemos um grupo bisimplicial cuja diagonal é

$$
\cdots \underset{\Longrightarrow}{\Longrightarrow}\left(V_{3} \times V_{3} \times V_{3}\right) \rtimes W_{3} \underset{\equiv}{\Longrightarrow}\left(V_{2} \times V_{2}\right) \rtimes W_{2}^{\prime} \Longrightarrow V_{1} \rtimes W_{1}^{\prime} \Longrightarrow W_{0}
$$

e o complexo de Moore dele é um complexo de grupos. Existe um morfismo desse complexo ao descrito anteriormente dado por

$$
\left(w ; v 1, v 2, \ldots, v_{n}\right) \mapsto\left(w, d_{n}^{V}\left(v_{n}\right)\right) .
$$

Começando com um cat $^{n}$-grupo simplicial esse procedimento nos da um morfismo de complexos de cat ${ }^{n-1}$-grupos. Considere $G$ como um cat $^{n}$-grupo simplicial trivial, ou seja ele é apenas não trivial em dimensão 0 , aonde ele é $G$. Se para cada $0 \leq k<n$ aplicarmos o funtor $\mathcal{N}_{1+k} \ldots \mathcal{N}_{n}$ em $G$ obtemos um cat ${ }^{n-k}$-grupo $(k+1)$-simplicial. Se $k=n$ simplesmente mantemos o cat ${ }^{n}$-grupo simplicial $G$. Agora se aplicarmos o método dos cones no complexo de Moore da diagonal obtemos um complexo de grupos $C_{\bullet}^{k}(G)$, sendo que sempre temos um morfismo de complexo de grupos de $C_{\bullet}^{k}(G)$ a $C_{\bullet}^{k+1}(G)$. Note também que $C_{\bullet}^{0}(G)=C_{\bullet}^{M}(G)$ e $C_{\bullet}^{n}(G)=C_{\bullet}(G)$. Temos portanto uma sequencia de $n$ morfismos entre complexos de grupos tal que o primeiro é $C_{\bullet}^{M}(G)$ e o último é $C \bullet(G)$. Definimos $\varepsilon(G)$ como a composição desses morfismos.

\footnotetext{
${ }^{1}$ Diagram chasing
} 
Para ver que esse morfismo necessariamente é um quasi-isomorfismo note primeiro que se

$$
1 \rightarrow G^{\prime} \rightarrow G \rightarrow G^{\prime \prime} \rightarrow 1
$$

é uma sequência exata curta de cat ${ }^{n}$-grupos e se dois dos morfismos $\varepsilon\left(G^{\prime}\right), \varepsilon(G)$ e $\varepsilon\left(G^{\prime \prime}\right)$ são quasiisomorfismos então o terceiro também o é como consequência do lema dos cinco.

Agora veja que se $G$ é da forma $M \rtimes M \operatorname{com} s_{i}\left(m, m^{\prime}\right)=m^{\prime}$ e $t_{i}\left(m, m^{\prime}\right)=m m^{\prime}$ para algum $i \in\langle n\rangle$ então $\varepsilon(G)$ é um quasi-isomorfismo já que $B(G)$ é contrátil e $C_{\bullet}(G)$ é acíclico.

Se $G$ é tal que para algum $i \in\langle n\rangle$ temos que $s_{i}=\mathbb{1}_{G}=t_{i}$ então $\varepsilon(G)$ é um quasi-isomorfismo já que nesse caso tanto $B(G)$ quanto $C_{\bullet}(G)$ podem ser obtidos ignorando a estrutura categorial relacionada a $i$, o que nos traz para o caso da hipótese de indução.

Da sequencia exata curta

$$
1 \rightarrow \operatorname{Ker} s_{i} \rtimes \operatorname{Ker} s_{i} \rightarrow \Gamma_{n}^{0}(G) \rightarrow \bar{\Gamma}_{n}^{0}(G) \rightarrow 1
$$

e dos últimos três parágrafos segue que $\varepsilon\left(\Gamma_{n}^{0}(G)\right)$ é um quasi-isomorfismo.

Como $\Gamma_{n}^{-1}(G)$ é tal que $s_{n}=\mathbb{1}=t_{n}$ temos que $\varepsilon\left(\Gamma_{n}^{-1}(G)\right)$ é um quasi-isomorfismo. Isso, o último paragrafo mais a sequencia exata curta

$$
1 \rightarrow \Gamma_{n}^{-1}(G) \rightarrow \Gamma_{n}^{0}(G) \rightarrow G \rightarrow 1
$$

nos dão que $\varepsilon(G)$ é um quasi-isomorfismo

Corolario 3.4.8. A categoria homotópica dos $(n+1)$-tipos homotópicos conexos é equivalente à categoria da localização dos cat $^{n}$-grupos obtida invertendo os quasi-isomorfismos.

Demonstração: Pelo teorema 3.4.7 e o teorema de Whitehead temos que o funtor $B$ leva quasi-isomorfismos a equivalências homotópicas, logo pela propriedade universal das localizações $B$ está bem definido nessa localização. Notando que $B$ pode ser pensado como a composição de $\mathcal{B}$ com o funtor $F$ da seção $\mathbf{2 . 2}$ é fácil ver, usando os resultados vistos até agora, que seu inverso é dado pela composição do funtor $G$ da seção 2.2 com o funtor $\Pi$ da seção 3.2 .

\subsection{Teorema de Seifert-van Kampen generalizado}

Agora provaremos que o funtor $\Pi$ descrito na seção 3.2 preserva colimites de certos diagramas, resultado provado originalmente por Brown e Loday em [BL87a]. Esse teorema é o que nos permitirá usar os cálculos de colimites do capitulo 1 nas aplicações do próximo capítulo.

Para todo conjunto não-vazio $\Lambda$ denote por $\Lambda_{\text {fin }}$ a categoria dos subconjuntos finitos de $\Lambda$, cujos morfismos são as inclusões. Seja $U=\left\{U_{\lambda}\right\}_{\lambda \in \Lambda}$ um recobrimento por abertos de um espaço pontuado $X$ tal que cada $U_{\lambda}$ contém o ponto base. Se $\sigma$ é um subconjunto finito não-vazio de $\Lambda$, então $U_{\sigma}$ denota as intersecções dos $U_{\lambda}$ para $\lambda \in \sigma$. Se $\sigma \subseteq \tau$, então $U_{\tau} \subseteq U_{\sigma}$, e portanto $U$ determina um funtor contravariante de $\Lambda_{\text {fin }}$ na categoria dos espaços que manda a inclusão $\sigma \hookrightarrow \tau$, na inclusão $U_{\tau} \hookrightarrow U_{\sigma}$. Podemos falar do colimite do diagrama resultante, ou seja da imagem desse funtor, como descrito em [Mac98],

$$
\underset{\sigma \in \Lambda_{\text {fin }}}{\operatorname{colim}} U_{\sigma}
$$

assim como 


$$
\underset{\sigma \in \Lambda_{\text {fin }}}{\operatorname{colim}} \phi\left(U_{\sigma}\right)
$$

para qualquer funtor covariante $\phi$ definido nos $U_{\sigma}$.

Provaremos agora a generalização do teorema de Seifert-van Kampen para o funtor covariante $\Pi$, cuja prova será feita por indução usando o teorema clássico para o caso $n=0$.

Teorema 3.5.1 (Teorema de Seifert-van Kampen para $n$-cubos fibrantes). Seja X um n-cubo fibrante e seja $\left\{U_{\lambda}\right\}_{\lambda \in \Lambda}$ um recobrimento por abertos de $X_{\langle n\rangle}$ tal que todos os abertos contém o ponto base. Cada $U_{\sigma}$ para $\sigma \in \Lambda_{\text {fin }}$ determina por imagem inversa um n-cubo fibrante que também denotaremos por $U_{\sigma}$. Suponha que cada $U_{\sigma}$ é um n-cubo fibrante conexo. Então

(C) o n-cubo fibrante $X$ é conexo;

(I) o homomorfismo natural de cat ${ }^{n}$-grupos

$$
\underset{\sigma \in \Lambda_{\text {fin }}}{\operatorname{colim}} \Pi\left(U_{\sigma}\right) \rightarrow \Pi(X)=\Pi\left(\underset{\sigma \in \Lambda_{\text {fin }}}{\operatorname{colim}} U_{\sigma}\right)
$$

é um isomorfismo.

Provaremos o teorema por indução em $n$. Denotemos por $\left(\mathbf{C}_{n}\right)$ e $\left(\mathbf{I}_{n}\right)$ a afirmação de que a conectividade dos $n$-cubos $U_{\sigma}\left(\sigma \in \Lambda_{\text {fin }}\right)$ implica a conectividade e isomorfismo de 3.5.1.C e 3.5.1.I respectivamente. Note que $\left(\mathbf{C}_{0}\right)$ e $\left(\mathbf{I}_{0}\right)$ são o teorema clássico de Seifert-van Kampen para o grupo fundamental. Provaremos $\left(\mathbf{C}_{n}\right)$ e $\left(\mathbf{I}_{n}\right)$ assumindo $\left(\mathbf{C}_{n-1}\right)$ e $\left(\mathbf{I}_{n-1}\right)$

Demonstração de (C): Precisamos de um lema:

Lema 3.5.2. Seja $X$ um n-cubo fibrante. Então são equivalentes:

i) $X$ é conexo.

ii) $\breve{\partial}_{i}^{-}(X)$ e $\breve{\partial}_{i}^{+}(X)$ são conexos para todo $i \in\langle n\rangle$ e a aplicação induzida de cat ${ }^{(n-1)}$-grupos

$$
\Pi \circ \mathrm{\partial}_{n}^{-}(X) \rightarrow \Pi \circ \mathrm{\partial}_{n}^{+}(X)
$$

é sobrejetora no grupo subjacente.

Demonstração: Denote por $F$ a fibra da aplicação

$$
p_{\langle n\rangle}: X_{\emptyset} \rightarrow \underset{S \neq \emptyset}{\operatorname{holim}} X_{S}
$$

Então $X$ é conexo se, e somente se, $F, \widetilde{\partial}_{i}^{-}(X)$ e $\widetilde{\partial}_{i}^{+}(X)$ forem conexos para todo $i \in\langle n\rangle$. Podemos ver que $F$ também é a fibra do morfismo no topo do diagrama abaixo, aonde os morfismos verticais são sequências de fibrações

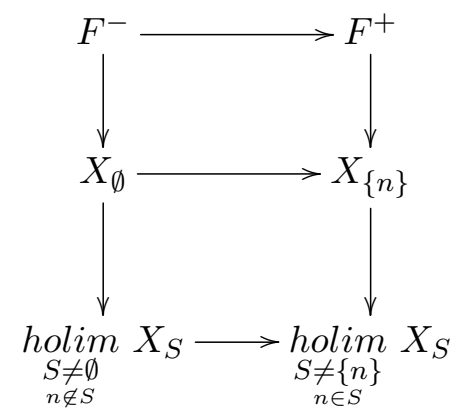


mas como o limite homotópico de um diagrama de fibrações é apenas o limite usual temos que esse morfismo é simplesmente

$$
\left(\operatorname{Simp} \circ \partial_{n}^{-}(X)\right)_{1 \ldots 1} \rightarrow\left(\operatorname{Simp} \circ \partial_{n}^{+}(X)\right)_{1 \ldots 1}
$$

Logo o lema segue da sequencia exata homotópica dessa fibração e da definição de $\Pi$

Pela hipótese de indução $\left(\mathbf{I}_{n-1}\right)$ sabemos que

$$
\Pi \circ \mathrm{\partial}_{i}^{ \pm}(X) \cong \operatorname{colim}_{\sigma \in \Lambda_{\mathrm{fin}}} \Pi \circ \mathrm{\partial}_{i}^{ \pm}\left(U_{\sigma}\right)
$$

Como $U_{\sigma}$ é conexo a aplicação $\Pi \circ{\partial_{n}^{-}}_{n}^{-}\left(U_{\sigma}\right) \rightarrow \Pi \circ \widetilde{\partial}_{n}^{+}\left(U_{\sigma}\right)$ é sobrejetora pelo lema 3.5.2, e portanto a aplicação no colimite é sobrejetora. Pela hipótese $\left(\mathbf{C}_{n-1}\right)$ temos que $\widetilde{\partial}_{i}^{ \pm}(X)$ é conexo, logo pelo Lema 3.5.2 novamente $X$ é conexo. Logo está demonstrado $\left(\mathbf{C}_{n}\right)$ isomorfismos:

Demonstração de (I): O isomorfismo $\operatorname{colim}_{\sigma \in \Lambda_{\mathrm{fin}}} \Pi\left(U_{\sigma}\right) \cong \Pi\left(\underset{\sigma \in \Lambda_{\mathrm{fin}}}{\operatorname{colim}} U_{\sigma}\right)$ é uma composição de vários

$$
\begin{aligned}
& \operatorname{colim}_{\sigma \in \Lambda_{\text {fin }}} \Pi\left(U_{\sigma}\right) \stackrel{(\mathbf{a})}{\cong} \operatorname{ass}\left(\underset{\sigma \in \Lambda_{\text {fin }}}{\operatorname{colim}} \Pi\left(U_{\sigma}\right)\right) \\
& \stackrel{\text { def }}{=} \text { ass }\left(\underset{\sigma \in \Lambda_{\text {fin }}}{\operatorname{colim}}\left(\wedge \circ \pi_{1} \circ \operatorname{Simp}\left(U_{\sigma}\right)\right)\right) \\
& \stackrel{(\text { b) }}{\cong} \text { ass } \circ \wedge \circ \pi_{1}\left(\underset{\sigma \in \Lambda_{\text {fin }}}{\operatorname{colim}} \operatorname{Simp}\left(U_{\sigma}\right)\right) \\
& \stackrel{(\mathbf{c})}{\cong} \text { ass } \circ \wedge \circ \pi_{1} \circ \operatorname{Simp}\left(\operatorname{colim}_{\sigma \in \Lambda_{\text {fin }}} U_{\sigma}\right) \\
& \stackrel{(\text { d) }}{\cong} \wedge \circ \pi_{1} \circ \operatorname{Simp}\left(\underset{\sigma \in \Lambda_{\text {fin }}}{\operatorname{colim}} U_{\sigma}\right) \\
& \stackrel{\text { def }}{=} \Pi\left(\underset{\sigma \in \Lambda_{\text {fin }}}{\operatorname{colim}} U_{\sigma}\right) \text {. }
\end{aligned}
$$

Note que vimos na seção 3.2 que vale o isomorfismo (d). O isomorfismo (a) segue do lema abaixo.

Lema 3.5.3. Se $\left(G_{\sigma}\right)_{\sigma \in \Sigma}$ é um diagrama de cat ${ }^{n}$-grupos, então

$$
\underset{\sigma \in \Sigma}{\operatorname{colim}} G_{\sigma} \cong \operatorname{ass}\left(\operatorname{colim}_{\sigma \in \Sigma} G_{\sigma}\right)
$$

Demonstração: Note que o grupo subjacente do pré-cat ${ }^{n}$-grupo $\operatorname{colim}_{\sigma \in \Sigma} G_{\sigma}$ é simplesmente o colimite, na categoria dos grupos, dos grupos subjacentes dos $G_{\sigma}$. Como esse é o cocone universal na categoria dos pré-cat ${ }^{n}$-grupos e um homomorfismo de pré-cat ${ }^{n}$-grupos necessariamente leva os elementos do Ker $s_{i}$ e do Ker $t_{i}$ do domínio no Ker $s_{i}$ e no Ker $t_{i}$ do contradomínio, então os kerneis de homomorfismos de um pré-cat ${ }^{n}$-grupo em um cat ${ }^{n}$-grupo contém os elementos de [Ker $s_{i}$, Ker $t_{i}$ ], logo o quociente de $\operatorname{colim}_{\sigma \in \Sigma} G_{\sigma}$ pelos subgrupos normais [Ker $s_{i}$, Ker $t_{i}$ ] é um cocone universal na categoria de cat $^{n}$-grupos

Para (b) precisamos de

Lema 3.5.4. Se o n-cubo $U_{\sigma}$ é conexo para todo $\sigma \in \Lambda_{\text {fin }}$, então o morfismo natural 


$$
\underset{\sigma \in \Lambda_{\text {fin }}}{\operatorname{colim}}\left(\wedge \circ \pi_{1} \circ \operatorname{Simp}\left(U_{\sigma}\right)\right) \rightarrow \wedge \circ \pi_{1}\left(\underset{\sigma \in \Lambda_{\text {fin }}}{\operatorname{colim}} \operatorname{Simp}\left(U_{\sigma}\right)\right)
$$

é um isomorfismo.

Demonstração: Devido a funtorialidade esse morfismo necessariamente comuta com os endomorfismos de grupos das estruturas de cat ${ }^{n}$-grupos, portanto basta provar esse isomorfismo para os grupos subjacentes:

$$
\underset{\sigma \in \Lambda_{\text {fin }}}{\operatorname{colim}}\left(\pi_{1}\left(\left(\operatorname{Simp}\left(U_{\sigma}\right)\right)_{1 \ldots 1}\right)\right)=\pi_{1}\left(\underset{\sigma \in \Lambda_{\text {fin }}}{\operatorname{colim}}\left(\operatorname{Simp}\left(U_{\sigma}\right)\right)_{1 \ldots 1}\right)
$$

Note que os espaços $V_{\sigma}=\left(\operatorname{Simp}\left(U_{\sigma}\right)\right)_{1 \ldots 1}\left(\sigma \in \Lambda_{\text {fin }}\right)$ podem ser identificados com subespaços abertos de $(\operatorname{Simp}(X))_{1 \ldots 1}$ e que $V_{\sigma}=\bigcap_{\lambda \in \sigma} V_{\lambda}$. Logo o colimite dos $V_{\sigma}$ é a sua união. Portanto a equalidade acima segue do teorema de Seifert-van Kampen clássico desde que os $V_{\sigma}$ sejam conexos para todos os $\sigma \in \Lambda_{\text {fin }}$.

Por hipótese $U_{\sigma}$ é conexo, logo $\mathscr{S}_{n} U_{\sigma}$ é um objeto simplicial de $(n-1)$-cubos fibrados conexos. Continuando esse processo $n-1$ vezes obtemos Simp $U_{\sigma}$ que portanto é um espaço n-simplicial conexo. Em particular $V_{\sigma}$ é conexo

O isomorfismo (c) é obtido aplicando o funtor $\mathbf{a s s}^{\wedge} \circ \pi_{1}$ à aplicação

$$
E=\underset{\sigma \in \Lambda_{\text {fin }}}{\operatorname{colim}} \operatorname{Simp}\left(U_{\sigma}\right) \rightarrow E^{X}=\operatorname{Simp}\left(\underset{\sigma \in \Lambda_{\text {fin }}}{\operatorname{colim}} U_{\sigma}\right)
$$

Novamente por funtorialidade basta checar o isomorfismo nos grupos subjacentes. Pelo lema 3.5.3 o grupo subjacente de $\operatorname{ass}^{\wedge} \circ \pi_{1}(E)$ é o quociente de $\pi_{1}\left(E_{1 \ldots 1}\right)$ pelos subgrupos $\left[\operatorname{Ker} s_{i}\right.$, Ker $\left.t_{i}\right](i \in$ $\langle n\rangle)$. Portanto precisamos apenas mostrar que $\pi_{1}\left(E_{1 \ldots 1}^{X}\right)$ é obtido da mesma forma, uma vez que, como vimos na seção 3.2, o funtor ${ }^{\wedge}$ leva os grupos $n$-simpliciais a um pré-cat ${ }^{n}$-grupo.

Para usar a hipótese de indução nós introduzimos um $n$-espaço simplicial $E^{\prime}$ entre $E$ e $E^{X}$ como no diagrama

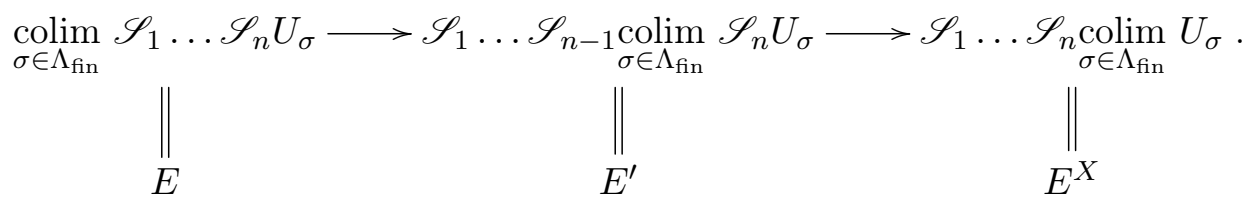

Por indução $\pi_{1}\left(E_{1 \ldots 1}^{\prime}\right)$ é igual ao quociente de $\pi_{1}\left(E_{1 \ldots 1}\right)$ pelos subgrupos [Ker $s_{i}$, Ker $\left.t_{i}\right](i \in\langle n-1\rangle)$. Logo o isomorfismo (c) segue do lema 3.5.7 abaixo, que por sua vez segue dos próximos dois lemas.

Lema 3.5.5. Seja $E_{\bullet}$ um espaço simplicial conexo ( $E_{m}$ conexo para todo $\left.m\right)$. Então existe uma sequencia exata de grupos

$$
\pi_{0}\left(D \circ \mathcal{N}\left(\pi_{2}\left(E_{\bullet}\right)\right)\right) \rightarrow \pi_{2}\left(\left|E_{\bullet}\right|\right) \rightarrow\left(N \pi_{1}\left(E_{\bullet}\right)\right)_{1} / \operatorname{Im} \delta_{2} \rightarrow \pi_{1}\left(E_{0}\right) \rightarrow \pi_{1}\left(\left|E_{\bullet}\right|\right)
$$

Demonstração: Sebe-se que a qualquer espaço simplicial conexo $E_{\bullet}$ está associado uma sequencia espectral ${ }^{2}$

$$
E_{p q}^{2}=\pi_{p}\left(D \circ \mathcal{N}\left(\pi_{q}\left(E_{\bullet}\right)\right)\right) \Rightarrow \pi_{p+q}\left(\left|E_{\bullet}\right|\right)
$$

A conexidade dos espaços $E_{n}$ implicam que a primeira fileira não trivial no plano $E^{2}$ é a com $q=1$. Isso nos dá $\pi_{0}\left(D \circ \mathcal{N}\left(\pi_{1}\left(E_{\bullet}\right)\right)\right)=\pi_{1}\left(\left|E_{\bullet}\right|\right)$ e a sequencia exata

$$
\pi_{0}\left(D \circ \mathcal{N}\left(\pi_{2}\left(E_{\bullet}\right)\right)\right) \rightarrow \pi_{2}\left(\left|E_{\bullet}\right|\right) \rightarrow H_{1}\left(N \pi_{1}\left(E_{\bullet}\right)\right) \rightarrow 1
$$

\footnotetext{
${ }^{2}$ Teorema A.2 no apêndice por Michel Zisman em [EM10].
} 
para computar $H_{p}\left(N \pi_{1}\left(E_{\bullet}\right)\right)$ consideramos o complexo de Moore do grupo simplicial $\pi_{1}\left(E_{\bullet}\right)$

$$
\left(N \pi_{1}\left(E_{\bullet}\right)\right)_{2} \stackrel{\delta_{2}}{\longrightarrow}\left(N \pi_{1}\left(E_{\bullet}\right)\right)_{1} \stackrel{\delta_{1}}{\rightarrow}\left(N \pi_{1}\left(E_{\bullet}\right)\right)_{0}=\pi_{1}\left(E_{0}\right) .
$$

A sequencia espectral nos dá então a sequencia exata

$$
1 \rightarrow H_{1}\left(N \pi_{1}\left(E_{\bullet}\right)\right) \rightarrow\left(N \pi_{1}\left(E_{\bullet}\right)\right)_{1} / \operatorname{Im} \delta_{2} \rightarrow \pi_{1}\left(E_{0}\right) \rightarrow \pi_{0}\left(D \circ \mathcal{N}\left(\pi_{1}\left(E_{\bullet}\right)\right)\right) \rightarrow 1 .
$$

Juntando (3.1) e (3.2) obtemos o resultado buscado

Lema 3.5.6. Seja $f: W \rightarrow Z$ uma fibração conexa e seja $E_{\bullet} \rightarrow E_{\bullet}^{f}$ uma aplicação de espaços simpliciais satisfazendo

a) E• é conexo;

b) $E_{\bullet} \rightarrow E_{\bullet}^{f}$ é uma equivalência homotópica fraca;

c) $\left|E_{\bullet}\right| \rightarrow\left|E_{\bullet}^{f}\right|$ é uma equivalência homotópica fraca;

d) $\pi_{1}\left(E_{2}\right)$ é gerado por elementos degenerados.

Então $\pi_{1}\left(E_{1}^{f}\right)=\pi_{1}\left(E_{1}\right) /\left[\right.$ Ker s,Ker b] aonde $s$ e $t$ são composições das aplicações de bordo $\partial_{1}$ e $\partial_{0}$ com a degeneração $d_{0}$.

Demonstração: Pelo lema 3.5.5 e as hipóteses sobre $E_{\bullet}$ e $E_{\bullet}^{f}$ temos o diagrama comutativo de sequencias exatas

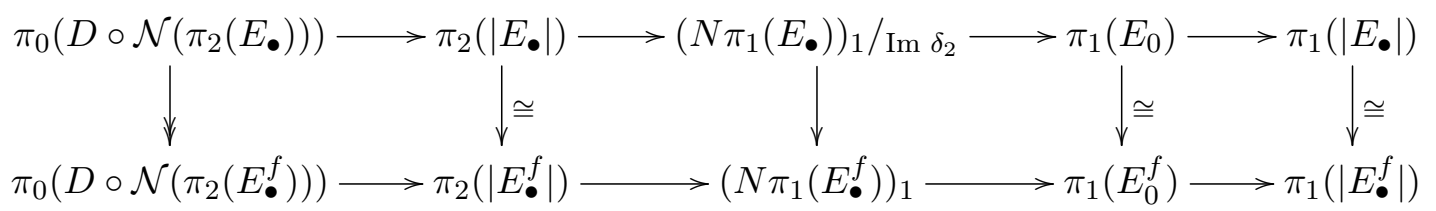

Aqui usamos o fato que para $E_{\bullet}^{f}$ o grupo $\operatorname{Im} \delta_{2}$ é trivial, como vimos no lema 3.2.2. Os isomorfismo verticais seguem de $b$ ) e c). De b) também concluímos que $\pi_{0}\left(D \circ \mathcal{N}\left(\pi_{2}\left(E_{\bullet}\right)\right)\right) \rightarrow \pi_{0}\left(D \circ \mathcal{N}\left(\pi_{2}\left(E_{\bullet}^{f}\right)\right)\right)$ é sobrejetora. Pelo lema dos cinco deduzimos que $\left(N \pi_{1}\left(E_{\bullet}\right)\right)_{1} / \operatorname{Im} \delta_{2} \rightarrow\left(N \pi_{1}\left(E_{\bullet}^{f}\right)\right)_{1}$ é um isomorfismo, logo por $\pi_{1}\left(E_{0}\right) \cong \pi_{1}\left(E_{0}^{f}\right)$ temos que $\pi_{1}\left(E_{1}\right) / \operatorname{Im} \delta_{2} \rightarrow \pi_{1}\left(E_{1}^{f}\right)$ é um isomorfismo. A condição $d$ ) e o lema 3.2.3 implicam que $\operatorname{Im} \mu_{2}=[\operatorname{Ker} s, \operatorname{Ker} b]$, do que segue a proposição.

Lema 3.5.7. O grupo $\pi_{1}\left(E_{1 \ldots 1}^{X}\right)$ é obtido tomando o quociente de $\pi_{1}\left(E_{1 \ldots 1}^{\prime}\right)$ por $\left[\right.$ Ker $\left.s_{n}, \operatorname{Ker} b_{n}\right]$, aonde $s_{n}$ e $t_{n}$ são composições das aplicações de bordo $\partial_{0}^{n}$ e $\partial_{1}^{n}$ com a degeneração $d_{0}^{n}$ na direção $n$.

Demonstração: Isso é uma consequência direta do lema 3.5.6 aplicado ao morfismo de espaços simpliciais $E_{1 \ldots 1}^{\prime} \bullet \rightarrow E_{1 \ldots 1 \bullet}^{X}$, uma vez que verificarmos as hipóteses $\left.a\right)$-d).

Como podemos pensar em $X$ como uma fibração de $(n-1)$-cubos fibrantes $\phi_{n}: \check{\partial}_{n}^{-}(X) \rightarrow \partial_{n}^{+}(X)$, temos a aplicação induzida $\mathscr{S}_{1} \ldots \mathscr{S}_{n-1} \circ \phi_{n}$ de $(n-1)$-espaços simpliciais, e portanto um morfismo $f=\left(\mathscr{S}_{1} \ldots \mathscr{S}_{n-1} \circ \phi_{n}\right)_{1 \ldots 1}$ de espaços. Note que $E_{\bullet}^{f}=E_{1 \ldots 1 \bullet}^{X}$. É justamente na função $f$ que aplicamos lema 3.5.6. Note que, como provamos $\left(\mathbf{C}_{n}\right), f$ é conexa.

Os $(n-1)$-cubos simpliciais $\mathscr{S}_{n} U_{\sigma}$ são conexos. Logo os $(n-1)$-cubos $\left(\mathscr{S}_{n} U_{\sigma}\right)_{m}(m \geq 0)$ são conexos, e a hipótese de indução $\left(\mathbf{C}_{n-1}\right)$ nos dá que 


$$
\underset{\sigma \in \Lambda_{\text {fin }}}{\operatorname{colim}}\left(\mathscr{S}_{n} U_{\sigma}\right)_{m}
$$

é conexo. Aplicando $\mathscr{S}_{1} \ldots \mathscr{S}_{n-1}$ obtemos que $E^{\prime}$ é conexo, e portanto a hipótese a) é satisfeita.

A hipótese de indução $\left(\mathbf{I}_{n-1}\right)$ aplicada para $m=2$ nos dá que $\pi_{1} E_{1 \ldots 12}^{\prime}$ é um quociente de

$$
\pi_{1} E_{1 \ldots 12}=\operatorname{colim}_{\sigma \in \Lambda_{\text {fin }}} \pi_{1} E_{1 \ldots 12}^{U_{\sigma}}
$$

Pelo lema 3.2.2 $\pi_{1} E_{1 \ldots 12}^{U_{\sigma}}$ é gerado por elementos degenerados (na direção $n$ ). Logo o colimite $\pi_{1} E_{1 \ldots 12}$ também o é, e portanto também o quociente $\pi_{1} E_{1 \ldots 12}^{\prime}$. Essa é a hipótese $d$ ).

Existem equivalências homotópicas fracas de $(n-1)$-cubos

$$
\left(\mathscr{S}_{n} X\right)_{0} \rightarrow \partial_{1}^{-}(X) \text { e }\left(\mathscr{S}_{n} U_{\sigma}\right)_{0} \rightarrow \partial_{1}^{-}(U)_{\sigma}
$$

Logo temos o diagrama comutativo

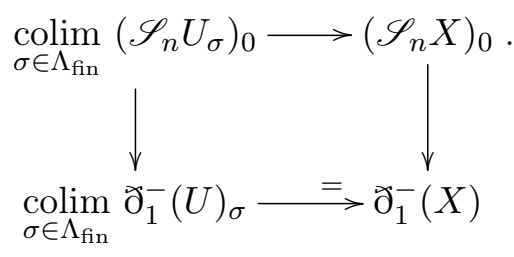

A aplicação da esquerda é um colimite de equivalências homotópicas fracas, logo é uma equivalência homotópica fraca ${ }^{3}$. Logo a aplicação de cima é uma equivalência homotópica fraca. Aplicando $\mathscr{S}_{1} \ldots \mathscr{S}_{n-1}$ obtemos uma equivalência homotópica fraca de espaços, o que nos dá a hipótese $b$ ).

Como simplicialização (ou seja a aplicação de $\mathscr{S}_{i}$ ) comuta com a realização geométrica basta, para obtermos a hipótese $c$ ), demonstra-la para o caso $n=1$. Usamos o fato que para uma fibração conexa $g: W \rightarrow Z$, a aplicação natural $\left|E_{\bullet}^{g}\right| \rightarrow Z$ é uma equivalência homotópica fraca ${ }^{4}$. Aplicamos esse resultado em $f$ e $f_{\sigma}$ no diagrama abaixo

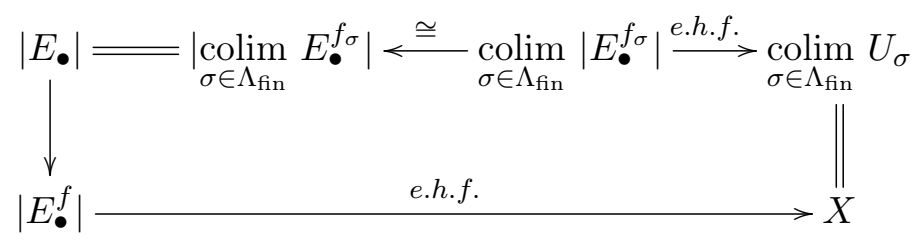

para provar que $\left|E_{\bullet}\right| \rightarrow\left|E_{\bullet}^{f}\right|$ é uma equivalencia homotopica fraca.

E assim completamos a demonstração de $\left(\mathbf{I}_{n}\right)$.

\section{6 -Cubo cruzado de grupos fundamental de um n-cubo de fibrações}

Dada a equivalência entre $n$-cubos cruzados de grupos e cat ${ }^{n}$-grupos da seção $\mathbf{1 . 3}$ temos que existe um funtor $n$-Cubo cruzado de grupos fundamental de um $n$-cubo de fibrações, que denotamos $\Pi$, associado ao funtor $\Pi$ da seção 3.2. Como mencionamos antes é esse o funtor que utilizaremos para nossas aplicações, já que podemos utilizar os colimites calculados no capítulo 1 .

Acontece que o funtor $\Pi$ tem uma definição razoavelmente transparente, analoga à definição do módulo cruzado fundamental de uma fibração dada por Whitehead, sem necessidade de métodos simpliciais como na definição do funtor П. A única parte problemática é definir o que faz o papel das funções $h$, que é dado por um produto generalizado de Whitehead.

\footnotetext{
${ }^{3}$ Proposição A.4 no apêndice por Michel Zisman em [EM10].

${ }^{4}$ Proposição A.5 no apêndice por Michel Zisman em [EM10].
} 
Definição 3.6.1. O n-cubo cruzado fundamental $\Pi(\bar{X})$ de um $n$-cubo de fibrações $\bar{X}$ é composto pelos grupos $\Pi(\bar{X})_{A}=\pi_{1}\left(\bar{X}_{A, \emptyset}\right), \quad(A \subseteq\langle n\rangle)$, os homomorfismos de bordo $\mu_{j}: \pi_{1}\left(\bar{X}_{A, \emptyset}\right) \rightarrow$ $\pi_{1}\left(\bar{X}_{A \backslash\{j\}, \emptyset}\right)$ induzidos por $\bar{X}_{A, \emptyset} \rightarrow \bar{X}_{A \backslash\{j\}, \emptyset}$ e as funções $h: \pi_{1}\left(\bar{X}_{A, \emptyset}\right) \times \pi_{1}\left(\bar{X}_{B, \emptyset}\right) \rightarrow \pi_{1}\left(\bar{X}_{A \cup B, \emptyset}\right)$ dadas por $(a, b) \mapsto\left(\underset{i \in A \cap B}{\circ} \mu_{i}\right) a \otimes b$, aonde $\otimes$ é o produto generalizado de Whitehead.

Por exemplo para uma $(n+1)$-tupla pontuada $\mathcal{X}=\left(X_{\langle n\rangle} ; X_{1}, \ldots, X_{n}\right)$ e seu $n$-cubo de fibrações associado $\bar{X}$ temos $\Pi(\bar{X})_{A}=\pi_{|A|+1}\left(X_{A} ; X_{A \backslash\{i\}}: i \in A\right)$ para $A \subseteq\langle n\rangle$.

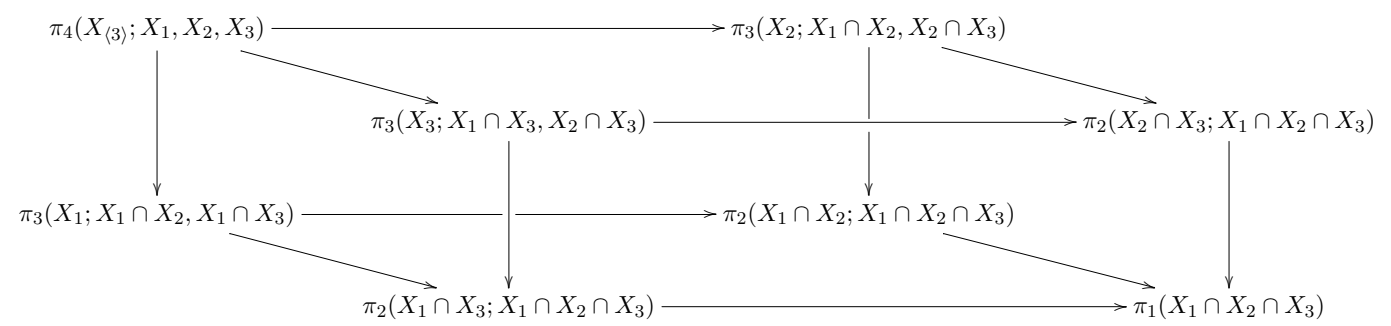

Figura 3.4: 3-cubo cruzado de grupos fundamental de uma quadrupla pontuada.

Precisamos portanto definir o que queremos dizer com produto generalizado de Whitehead. Seguindo as ideias de [BL87b, AS72] o produto generalizado de Whitehead será definido em uma classe de casos descrito abaixo que servirão como exemplos universais, e então o caso geral será dado por uma composição com esse exemplo universal.

Seja $I=[0,1]$. Defina $C=I^{n+1} / \sim$ aonde definimos que todos os pontos $\left(c_{1}, \ldots, c_{n+1}\right) \in I^{n+1}$ tais que $c_{i}=0$ para algum $i \in\langle n+1\rangle$ ou $c_{n+1}=1$ são equivalentes pela relação $\sim$. Defina agora $C_{i}=\left\{[c] \in C \mid c_{i}=1\right\}$ para todo $i \in\langle n\rangle$. Tomemos duas cópias $C^{1}$ e $C^{2}$ de $C$ vamos definir as $(n+1)$-tupla de espaços $\chi=\left(C^{1} \vee C^{2} ; C_{1}^{1} \vee C_{1}^{2}, \ldots, C_{n}^{1} \vee C_{n}^{2}\right)$ e $\chi^{i}=\left(C^{i} ; C_{1}^{i}, \ldots, C_{n}^{i}\right)$ para $i=1,2$. Temos então que

$$
\begin{aligned}
& \Pi\left(\chi^{i}\right)_{A}=\pi_{|A|+1}\left(\underset{k \in A}{\cap} C_{k}^{i} ; \underset{k \in A \cup\{l\}}{\cap} C_{k}^{1}, l \notin A\right)=\left\langle z_{A}^{i}\right\rangle \cong \mathbb{Z} \\
& \Pi(\chi)_{A}=\pi_{|A|+1}\left(\cap_{k \in A} C_{k}^{1} \vee C_{k}^{2} ; \underset{k \in A \cup\{l\}}{\cap} C_{k}^{1} \vee C_{k}^{2}, l \notin A\right)=\left\langle z_{A}^{1}, z_{A}^{2}\right\rangle \cong \mathbb{Z} * \mathbb{Z}
\end{aligned}
$$

Com os homomorfismos $\mu_{k}\left(z_{A}^{i}\right)=z_{A \backslash\{k\}}^{i}$ induzidos pelos morfismos de bordo. Nesse caso os produtos generalizados de Whitehead podem ser descritos explicitamente usando o product pairing descrito por Blakers e Massey em [BM53a]. Ele nos dá que $\left(z_{A}^{i} \otimes z_{B}^{j}\right)=\left[z_{A \cup B}^{i}, z_{A \cup B}^{j}\right]$, sendo que o produto de elementos arbitrários pode ser inferidos usando ( $\left.{ }^{n} \mathrm{CG} 8\right)$ e ( $\left.{ }^{n} \mathrm{CG} 9\right)$.

Não é difícil provar que $\Pi(\chi)$ é o coproduto de $\Pi\left(\chi^{1}\right)$ e $\Pi\left(\chi^{2}\right)$. Note que para todo $n$-cubo cruzado de grupos $G$ e $a \in G_{A}$ podemos definir um morfismo de $n$-cubos cruzados de grupos

$$
\begin{aligned}
& f_{a}^{i}: \Pi\left(\chi^{i}\right) \rightarrow G \\
& \left(f_{a}^{i}\right)_{A^{\prime}}\left(z_{A^{\prime}}^{i}\right)= \begin{cases}\underset{k \in A \backslash A^{\prime}}{\circ} \mu_{k}(a) & \text { se } A^{\prime} \subseteq A \\
1 & \text { c.c. }\end{cases}
\end{aligned}
$$

Logo podemos definir o produto generalizado de Whitehead da seguinte maneira. Seja $\bar{X}$ um $n$-cubo de fibrações. Definimos o produto generalizado de Whitehead para cada par $A, B \subseteq\langle n\rangle$ 
como sendo

$$
\begin{gathered}
\otimes: \pi_{1}\left(\bar{X}_{A, \emptyset}\right) \times \pi_{1}\left(\bar{X}_{B, \emptyset}\right) \rightarrow \pi_{1}\left(\bar{X}_{A \cup B, \emptyset}\right) \\
(a, b) \mapsto\left(f_{a}^{1} \circledast f_{b}^{2}\right)_{A \cup B}\left(\left[z_{A \cup B}^{1}, z_{A \cup B}^{2}\right]\right)
\end{gathered}
$$

Note que estamos usando o mesmo símbolo $\otimes$ para o produto generalizado de Whitehead e o produto tensorial não abeliano. Isso é para explicitar a relação entre essas duas operações que ficará clara pelas aplicações do capítulo 1. Ficará claro o que queremos dizer com esse símbolo pelo contexto. 



\section{Capítulo 4}

\section{Aplicações}

Veremos nesse capítulo algumas aplicações do teorema generalizado de Seifert-van Kampen. Começaremos nos focando em mostrando como os cálculos de módulos cruzados induzidos que fizemos na seção $\mathbf{1 . 5}$ podem ser usados para computar certos grupos de homotopia relativos e como ele nos dá a descrição de $\pi_{2}(X ; U)$ quando $X$ é obtido de $U$ colando 2-células, obtido pela primeira vez por Whitehead.

Na segunda seção mostramos como a teoria apresentada consegue demonstrar o teorema de Blakers-Massey sobre grupos de homotopia de triades com hipóteses sobre a tríade mais fracas que no resultado original.

A terceira seção apresenta um teorema de excisão para $(n+1)$-tuplas de espaços e mostraremos como esse resultado implica um teorema generalizado de Hurewicz e uma generalização da fórmula de Hopf para os grupos de homologia de um grupo. Vemos também como usar o teorema de excisão para descrever o efeito que colar 3-células tem no grupo de homotopia de uma tríade de espaços.

A quarta seção mostra como Ellis e Mikhailov obtiveram uma fórmula para os grupos de homotopia da esfera originalmente obtida por Wu usando outros métodos.

\subsection{Aplicações de módulos cruzados induzidos}

Veremos agora como o teorema 3.5.1 nos permite aplicar os resultados da seção $\mathbf{1 . 5}$ para computar certos módulos cruzados fundamentais. Relembrando a notação, dado um módulo cruzado $M=\left(\mu: M_{\{1\}} \rightarrow M_{\emptyset}\right)$, um grupo $G$ e um homomorfismo de grupos $f_{\emptyset}: M_{\emptyset} \rightarrow G$ temos o módulo cruzado induzido de $M$ por $f_{\emptyset}$ denotado por $f_{\emptyset *} M=\left(\mu: f_{\emptyset *} M_{\{1\}} \rightarrow G\right)$. Na seção $\mathbf{1 . 5}$ podem ser encontradas apresentações do grupo $f_{\emptyset *} M_{\{1\}}$ para diferentes hipóteses sobre $M, G$ e $f_{\emptyset}$.

Comecemos analisando como computar o módulo cruzado de um espaço que é a união de dois abertos.

Teorema 4.1.1. Suponha que $X$ é a união dos abertos conexos por caminhos $V$ e $A$, que $W=V \cap A$ é conexo por caminhos e que o par $(V ; W)$ é conexo. Então $(X ; A)$ é conexo e $\pi_{2}(X ; A) \rightarrow \pi_{1}(A)$ é o modulo cruzado induzido de $\pi_{2}(V ; W) \rightarrow \pi_{1}(W)$ pelo homomorfismo $\pi_{1}(W) \rightarrow \pi_{1}(A)$.

Demonstração: Temos portanto os seguintes diagramas, sendo que o da esquerda está na categoria dos pares de espaços, o da direita na categoria dos módulos cruzados e temos que o da esquerda é mandado para o da direita por $\Pi$ (Note que $\pi_{2}(Z ; Z)=1$ para qualquer espaço $Z$ ).

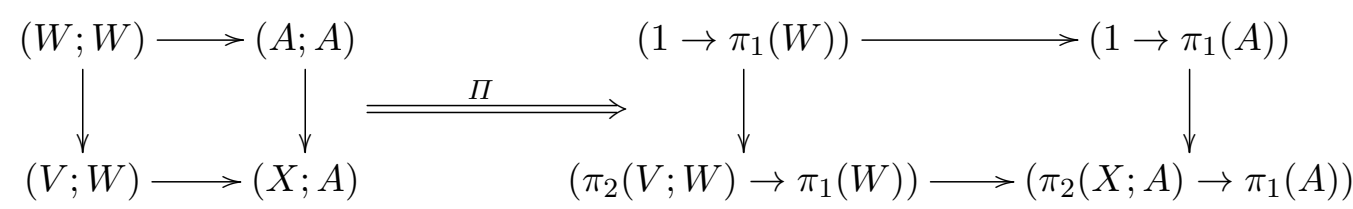


Como o diagrama da esquerda é um pushout que satisfaz as hipóteses do teorema 3.5.1 temos por esse teorema que o diagrama da direita também é um pushout. Logo o que qeríamos segue do teorema 1.5.4

Podemos usar esse resultado para computar o grupo de homotopia relativo de dimensão 2 do cone de uma aplicação $f: W \rightarrow A$, e até o grupo de homotopia absoluto caso $\pi_{2}(A)$ seja trivial.

Corolario 4.1.2. Sejam $W$ e A espaços conexos por caminhos e $\phi: W \rightarrow A$ uma cofibração fechada. Denote por $X=A \cup_{\phi} C W$ o cone de $\phi$ e por $f_{\emptyset}: \pi_{1}(W) \rightarrow \pi_{1}(A)$ o homomorfismo induzido por $\phi$. Então $(X ; A)$ é conexo e o módulo cruzado $\pi_{2}(X ; A) \rightarrow \pi_{1}(A)$ é isomorfo ao módulo cruzado induzido de $\pi_{2}(C W, W) \cong \pi_{1}(W) \rightarrow \pi_{1}(W)$, cujo morfismo é a identidade, pelo homomorfismo $f_{\emptyset}$. Em particular, se $\pi_{2}(A)=0$, então $\pi_{2}(X)$ é isomorfo ao kernel de $\mu: f_{\emptyset *} \pi_{1}(W) \rightarrow \pi_{1}(A)$

Demonstração: Esse resultado segue do teorema 4.1.1 já que para todo espaço $W$ temos que o par $(C W, W)$ é conexo e existem abertos $U_{1}$ e $U_{2}$ de $X$ tais que $C W \subset U_{1}$ e $A \subset U_{2}$, e $U_{1}$ retrai por deformação em $C W$ e $U_{2}$ retrai por deformação em $A$. O caso particular em que $\pi_{2}(A)=0$ segue da sequência exata do par.

Como consequência direta do último corolário obtemos o resultado que o grupo de homotopia relativa $\pi_{2}(X ; A)$ de um espaço $X$ obtido a partir de um espaço $A$ colando 2-células pode ser descrito pelo conceito de módulos cruzados livre da definição 1.5.7, como foi provado pela primeira vez por Whitehead [Whi49].

Teorema 4.1.3 (Whitehead). Seja $X=A \cup\left\{e_{\lambda}^{2}\right\}_{\lambda \in \Lambda}$ obtido do espaço conexo por caminhos $A$ colando 2-células. Então o módulo cruzado $\pi_{2}(X ; A) \rightarrow \pi_{1}(A)$ é isomorfo ao módulo cruzado livre gerado pela função dada pelas aplicações de bordo.

$$
\begin{aligned}
\Lambda & \rightarrow \pi_{1}(A) \\
\lambda & \mapsto\left[\partial e_{\lambda}^{2}\right]
\end{aligned}
$$

Em particular, no caso em que $A$ é um disco e colamos uma 2-célula em seu bordo via a função identidade obtemos facilmente que $X$ é homotópico a esfera $\mathbb{S}^{2}$ e como $A$ é contratil temos que $\pi_{2}(X) \cong \pi_{2}(X ; A) \cong \mathbb{Z}$

\subsection{Teorema de Blakers-Massey}

Veremos agora como usar os resultados da seção $\mathbf{1 . 7}$ sobre $n$-cubos cruzados de grupos $(n-1)$ universais para computar certos grupos de homotopia de $(n+1)$-tuplas. Como consequência desse resultado e do teorema 3.5.1 temos uma versão do teorema de Blakers-Massey [BM53b] provado por Brown e Loday em [BL87b] com hipóteses mais fracas que as originais. Para isso precisaremos levar em conta o produto tensorial não-abeliano descrito na seção 1.6.

Vejamos o enunciado do teorema de Blakers-Massey original [BM51, BM52, BM53b] que nos dá informações sobre certos grupos de homotopia de tríades de espaços.

Teorema (Blakers-Massey). Seja X um espaço que é a união de subespaços abertos $U$ e $V$ tais que $U, V$ e $W=U \cap V$ sejam conexos por caminhos, que $W$ seja simplesmente conexo e que os pares $(U ; W)$ e $(V ; W)$ sejam respectivamente $(p-1)$-conexo e $(q-1)$-conexo para $p, q \geq 3$, ou seja $\pi_{i}(U, W)=1$ se $1 \leq i \leq p-1$ e $\pi_{j}(V, W)=1$ se $1 \leq j \leq q-1$. Então a tripla $(X ; U, V)$ é 
$(p+q)$-conexa e o homomorfismo

$$
\pi_{p}(U ; W) \otimes_{\mathbb{Z}} \pi_{q}(V ; W) \rightarrow \pi_{p+q+1}(X ; U, V)
$$

é um isomorfismo.

Como para toda tríade de espaços $(X ; U, V)$, com $W=U \cap V$, temos a sequência exata

$$
\cdots \rightarrow \pi_{k+1}(X ; U, V) \rightarrow \pi_{k}(U ; W) \rightarrow \pi_{k}(X ; V) \rightarrow \pi_{k}(X ; U, V) \rightarrow \cdots
$$

esse teorema nos dá informações em um certo intervalo de dimensões sobre obstruções para a excisão na homotopia relativa, o que investigaremos mais a fundo na próxima seção.

Veremos agora como podemos relaxar as hipóteses desse teorema para os casos em que a interseç̧ão $W$ não é necessariamente simplesmente conexa e em que podemos tomar $p=2$ ou $q=2$. Primeiro precisaremos de um resultado preliminar que será útil.

Teorema 4.2.1. Seja $\mathcal{X}=\left(X_{\langle n\rangle} ; X_{1}, \ldots, X_{n}\right)$ uma $(n+1)$-tupla pontuada. Se

$$
\mathcal{X}_{i}=\left(X_{i} ; X_{j} \cap X_{i}: j \neq i\right)
$$

são $n$-tuplas conexas, então $\mathcal{X}$ é conexo, $\Pi(X)$ é um n-cubo cruzado de grupos $(n-1)$-universal e portanto $\Pi(X)_{\langle n\rangle}$ tem uma apresentação dada pelo teorema 1.7.4.

Demonstração: Para uma $(n+1)$-tupla pontuada $\mathcal{X}=\left(X_{\langle n\rangle} ; X_{1}, \ldots, X_{n}\right)$ temos os $n$-cubos de espaços ${ }^{\hat{A}} X, \hat{A} \subseteq\langle n\rangle$, com $\left({ }^{\hat{A}} X\right)_{A}=X_{A \backslash \hat{A}}$. Note que se $k \in \hat{A}$ então $\left({ }^{\hat{A}} X\right)_{A} \rightarrow\left({ }^{\hat{A}} X\right)_{A \cup\{k\}}$ é a identidade, $\operatorname{logo}\left({ }^{A} \bar{X}\right)_{A, B}$ é homotópico a um ponto se $A \cap \hat{A} \neq \emptyset$. Portanto se $A \cap \hat{A} \neq \emptyset$ então $\Pi\left({ }^{\hat{A}} X\right)_{A}=1$. Note também que temos inclusões canônicas $\Pi\left({ }^{\hat{A}} X\right) \rightarrow \Pi(\hat{A} \backslash\{k\} X)$. Portanto o resultado segue dos teoremas $\mathbf{1 . 7 . 3}$ e $\mathbf{3 . 5 . 1}$

Vejamos agora como esse resultado implica o teorema de Blakers-Massey generalizado.

Teorema 4.2.2 (Blakers-Massey generalizado). Seja $X$ um espaço que é a união de subespaços $U$ e $V$ tais que $U, V$ e $W=U \cap V$ sejam conexos por caminhos, e que os pares $(U ; W)$ e $(V ; W)$ sejam respectivamente $(p-1)$-conexo e $(q-1)$-conexo para $p, q \geq 2$. Então a tripla $(X ; U, V)$ é $(p+q)$-conexa e o homomorfismo

$$
\pi_{p}(U ; W) \otimes \pi_{q}(V ; W) \rightarrow \pi_{p+q+1}(X ; U, V)
$$

é um isomorfismo.

Demonstração: Tome a $(p+q-1)$-tupla $\chi=(X ; U, \ldots, U, V, \ldots, V)$ com $q-1$ cópias de $U$ e $p-1$ cópias de $V$. Usando sequências exatas podemos provar que $\pi_{i}(U ; V, \ldots, V) \cong \pi_{i}(U ; V)$ e que $\pi_{i}(U ; U, \ldots, U, V, \ldots, V)=1$ para qualquer número não nulo de cópias dos espaços. Logo, por $(U ; W)$ ser $p$-conexo, se denotarmos $\chi_{U}=(U ; U, \ldots, U, W, \ldots, W)$ com $q-1$ cópias de $U$ e $p-1$ cópias de $W$, então temos que $\chi_{U}$ é conexo e o único grupo de homotopia não trivial de $\Pi\left(\chi_{U}\right)$ é $\Pi\left(\chi_{U}\right)_{\{q, \ldots q+p-2\}}=\pi_{p}(U ; W)$. Analogamente $\chi_{V}$ é conexo e o único grupo de homotopia não trivial de $\Pi\left(\chi_{V}\right)$ é $\Pi\left(\chi_{V}\right)_{\{1, \ldots q-1\}}=\pi_{q}(V ; W)$. Portanto o resultado segue diretamente do teorema 4.2.1.

Nos casos $p, q>2$ esse é o resultado de Blakers e Massey sem precisar assumir que $W$ é simplesmente conexo, pois nesse caso $\pi_{p}(U ; W) \rightarrow \pi_{1}(W)$ e $\pi_{q}(V ; W) \rightarrow \pi_{1}(W)$ são funções triviais 
e portanto $\pi_{p}(U ; W)$ e $\pi_{q}(V ; W)$ são grupos abelianos que agem trivialmente um no outro, logo pelo teorema 1.6.5 o produto tensorial no teorema é o produto tensorial abeliano usual. Porém nos casos em que $p$ ou $q$ é igual a 2 os grupos envolvidos podem ser não-abelianos e a ação pode ser não trivial, logo devemos considerar o produto tensorial mais geral.

Em particular podemos tomar $X=\mathbb{S}^{2}$ e $U$ e $V$ os hemisférios norte e sul respectivamente. $\mathrm{O}$ resultado acima nos dá que o 2-cubo cruzado associado a essa tríade é dado por

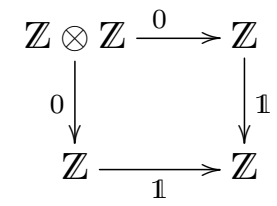

e que como $U$ e $V$ são contrateis é possível mostrar por sequencias exatas que $\pi_{3}\left(\mathbb{S}^{2}\right) \cong \pi_{3}(X ; U, V) \cong$ $\pi_{2}(U ; W) \otimes \pi_{2}(V ; W) \cong \mathbb{Z} \otimes \mathbb{Z} \cong \mathbb{Z}$.

\subsection{Excisão, teorema de Hurewicz e fórmulas de Hopf}

Mostraremos agora como em [BL87b] e [BE88] os resultados clássicos de excisão homotópica para pares de espaços, o teorema de Hurewicz e a fórmula de Hopf para o segundo grupo de homologia de um grupo foram generalizados. No final dessa seção também aplicamos o teorema de excisão no caso $n=2$ para descrever o terceiro grupo de homotopia de uma tríade de espaços $\left(X \cup \underset{\lambda \in \Lambda}{\vee} e_{\lambda}^{3} ; U, V\right)$ obtida colando 3-células em $X$.

Notemos que é fácil provar usando a sequência exata homotópica da tríade de espaços $(X ; U, V)$ que o teorema 4.2.2 da seção anterior implica o teorema de excisão homotópica clássico.

Teorema (Excisão). Seja $X$ um espaço que é a união dos abertos $U$ e $V$ tais que $U, V$ e $W=U \cap V$ sejam conexos por caminhos, e que os pares $(U ; W)$ e $(V ; W)$ sejam respectivamente $(p-1)$-conexo $e(q-1)$-conexo para $p, q \geq 2$. Então a inclusão $(U ; W) \hookrightarrow(X ; V)$ induz um homomorfismo

$$
\pi_{k}(U ; W) \rightarrow \pi_{k}(X ; V)
$$

que é um isomorfismo para $k<p+q-2$ e uma sobrejeção para $k=p+q-1$.

Veremos agora um teorema de excisão homotópica para $(n+1)$-tuplas de espaços que generaliza o que vimos sobre excisão para grupos de homotopia de pares de espaços. Usaremos os conceitos e resultados da seção 1.8 tanto para enunciar o teorema quanto na sua demonstração.

Teorema 4.3.1 (Excisão para ( $n+1)$-tuplas de espaços). Seja $X$ a união de abertos $U, V_{1}, \ldots, V_{n}$, e seja $W_{i}=U \cap V_{i}$ para $i \in\langle n\rangle$. Sejam $\chi=\left(U ; W_{1}, \ldots, W_{n}\right)$ e $\Upsilon=\left(X ; V_{1}, \ldots, V_{n}\right)$ tais que $\chi$ é conexo e $\left(V_{i} ; V_{j} \cap V_{i}: j \neq i\right)$ são conexos para todo $i \in\langle n\rangle$. Note que temos o morfismo de inclusão $\chi \hookrightarrow \Upsilon$. Então $\Upsilon$ é conexo e é o $n$-cubo cruzado de grupos $(n-1)$-induzido de $\Pi(\chi)$ por $i^{n-1}$, aonde $i^{n-1}$ é o morfismo de $(n-1, n)$ cantos cruzados de grupos associado ao morfismo $i: \Pi(\chi) \rightarrow \Pi(\Upsilon)$ induzido pela inclusão.

Demonstração: Podemos usar o recobrimento $\left\{X, V_{1}, \ldots, V_{n}\right\}$ de $Y$ de forma que o diagrama de $n$-cubos fibrantes determinado por esse recobrimento, como na hipótese do teorema 3.5.1, é levado pelo funtor $\Pi$ para um diagrama como o descrito nas hipóteses do teorema 1.8.2, logo o resultado segue dos dois teoremas mencionados. 
Podemos usar esse resultado para provar uma generalização do teorema de Hurewicz, que nos dá uma relação entre os grupos de homotopia de um espaço com os seus grupos de homologia.

Teorema (Hurewicz). Seja $X$ um espaço $(n-1)$-conexo. Então temos que $H_{k}(X)$ é trivial para todo $k=1, \ldots, n-1$ e para $k=n, n+1$ existe um homomorfismo

$$
\pi_{k}(X) \rightarrow H_{k}(X)
$$

que é sobrejetor se $k=n+1$, é um isomorfismo se $k=n \geq 2$ e é a abelianização se $k=n=1$.

Queremos generalizar a partir desse resultado uma relação entre os grupos de homotopia de $(n+1)$-tuplas de espaços com os grupos de homologia hiper-relativos que introduziremos agora. A ideia aqui é copiar uma construção da sequência exata da homologia de um par de espaços usando cones das funções inclusões. Seja $U$ um subespaço de $X$. Então temos que $H_{i}(X ; U) \cong H_{i}(X \cup C U)$, já que como cones são contrateis temos pela sequencia exata do par $H_{i}(X \cup C U) \cong H_{i}(X \cup C U ; C U)$ e por excisão da homologia obtemos $H_{i}(X ; U) \cong H_{i}(X \cup C U ; C U)$.

De fato temos que a sequência de espaços $U \hookrightarrow X \hookrightarrow X \cup C U$, por ser uma sequência de cofibrações, induz a sequência exata de homologia abaixo [May99, Cap. 14].

$$
\cdots \rightarrow H_{k}(U) \rightarrow H_{k}(X) \rightarrow H_{k}(X \cup C U) \rightarrow H_{k-1}(U) \rightarrow \cdots
$$

Seguindo a ideia dessa relação podemos portanto definir para uma $(n+1)$-tupla de espaços $\chi\left(X ; X_{1}, \ldots, X_{n}\right)$ os grupos de homologia hiper-relativos dados por $H_{i}\left(X ; X_{1}, \ldots, X_{n}\right)=H_{i}(X \cup$ $\left.C\left(X_{1} \cup \cdots \cup X_{n}\right)\right)$. Temos assim a seguinte generalização do teorema de Hurewicz.

Teorema 4.3.2 (Hurewicz para $(n+1)$-tuplas de espaços). Seja $\chi=\left(X ; X_{1}, \ldots, X_{n}\right)$ uma $(n+1)$ tupla de espaços tais que cada inclusão de $X_{i}$ em $X$ é uma cofibração fechada, e o n-cubo de fibrações associado é conexo. Então o espaço $Y=X \cup C\left(X_{1} \cup \cdots \cup X_{n}\right)$ é n-conexo e $H_{n+1}(Y)=$ $H_{n+1}\left(X ; X_{1}, \ldots, X_{n}\right)$ é isomorfo ao quociente de $\Pi(\chi)_{\langle n\rangle}=\pi_{n+1}\left(X ; X_{1}, \ldots, X_{n}\right)$ pelos subgrupos normais dados por $h\left(\Pi(\chi)_{B}, \Pi(\chi)_{C}\right)$ para $B, C \subseteq\langle n\rangle$ tais que $B \cup C=\langle n\rangle$.

Demonstração: Seja $v$ o vértice do cone em $Y=X \cup C\left(X_{1} \cup \cdots \cup X_{n}\right)$ e seja $X_{i}^{\prime}$ uma vizinhança aberta de $X_{i}$ em $X$ que retrai por deformação em $X_{i}$, que existe pela hipótese que suas inclusões são cofibrações fechadas [May99, Cap. 6, Teo. 1]. O recobrimento de $Y$ dado por $V=$ $\left\{X, C X_{1}, \ldots, C X_{n}\right\}$ determina um diagrama de $n$-cubos de fibrações homotopicamente equivalente ao diagrama dado pelo recobrimento por abertos $V^{\prime}=\left\{Y \backslash\{v\}, C X_{1} \cup X_{1}^{\prime}, \ldots, C X_{n} \cup X_{n}^{\prime}\right\}$, sendo que para o recobrimento $V^{\prime}$ vale o teorema 4.3.1 acima. Como para $\Upsilon=\left(Y ; C X_{1}, \ldots, C X_{n}\right)$ temos que o $(n-1)$-canto cruzado de grupos $\Pi(\Upsilon)^{n-1}$ é trivial, já que todo cone é contrátil, temos que a n-conectividade de $Y$ é dada pelo teorema 3.4.7 e a descrição de $H_{n+1}(Y)$ segue do fato que (Triv $\Pi(\chi))_{\langle n\rangle}=\pi_{n+1}\left(Y ; C X_{1}, \ldots, C X_{n}\right)$, do fato que $\pi_{n+1}\left(Y ; C X_{1}, \ldots, C X_{n}\right) \cong \pi_{n+1}(Y)$ e do teorema de Hurewicz clássico, já que por 3.4.7 temos que $\pi_{i}(Y) \cong \pi_{i}(B \circ \Pi(\Upsilon))$ é trivial para $i<n+1$.

Podemos agora mostrar como usar esses resultado para achar fórmulas para os grupos de homologia de dimensões arbitrarias de um grupo análogas à fórmula de Hopf para o segundo grupo de homologia. Daremos uma derivação para a fórmula de Hopf original pois esse caso serve como uma ilustração para a demonstração do caso geral.

Teorema (Fórmula de Hopf). Seja $F$ um grupo e $R$ um subgrupo normal tal que $H_{2}(F)=0$. Podemos por exemplo tomar $F$ um grupo livre. Então para $G=\frac{F}{R}$ temos que $H_{2}(G)=\frac{R \cap[F, F]}{[F, R]}$. 
Demonstração: Para derivar esse resultado note que o homomorfismo quociente $F \rightarrow \frac{F}{R}$ induz uma sequencia de cofibração $B(F) \rightarrow B\left(\frac{F}{R}\right) \rightarrow B\left(\frac{F}{R}\right) \cup C B(F)$, e o teorema 4.3.2 para $n=1$ nos dá que $H_{2}\left(B\left(\frac{F}{R}\right) ; B(F)\right)$ é isomorfo a $\pi_{2}\left(B\left(\frac{F}{R}\right) ; B(F)\right) \cong R$ quocientado pelo subgrupo normal $[F, R]$. A formula de Hopf segue então da sequencia exata de homologia.

$$
0 \rightarrow H_{2}(G) \rightarrow \frac{R}{[F, R]} \rightarrow \frac{F}{[F, F]} \rightarrow \frac{G}{[G, G]} \rightarrow 0
$$

Para usarmos o teorema de Hurewicz no estudo da homologia de grupos precisaremos construir $n$-cubos de fibrações a partir de $(n+1)$-tuplas de grupos. Seja $G$ um grupo, $\left\{N_{i}\right\}_{i \in\langle n\rangle}$ subgrupos normais de $G$ e $B$ o funtor que leva grupos ao seu espaço de classificação. Podemos construir um $n$-cubo de fibrações $B(\mathcal{G})$ associado a $(n+1)$-tupla de grupos $\mathcal{G}=\left(G ; N_{1}, \ldots, N_{n}\right)$ aonde

$$
B(\mathcal{G})_{A, B}=B\left(\frac{\bigcap_{a \in A}\left(\prod_{b \in B \cup\{a\}} N_{b}\right)}{\prod_{b \in B} N_{b}}\right)
$$

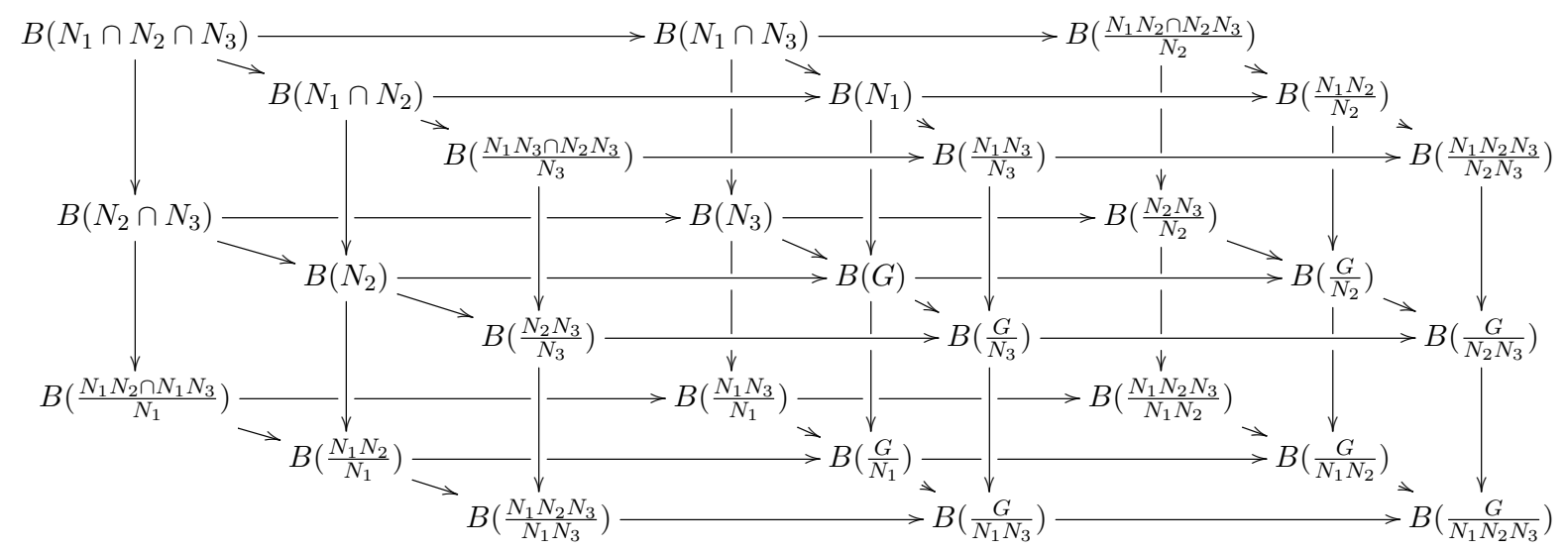

Figura 4.1: 3-cubo de fibrações associado aos subgrupos normais $N_{1}, N_{2}$ e $N_{3}$ de G.

Para podermos aplicar o teorema 3.5.1 a essa estrutura precisaremos de uma formulação algébrica de conectividade descrita no lema abaixo.

Lema 4.3.3. Seja $\mathcal{G}=\left(G ; N_{1}, \ldots, N_{n}\right)$ uma $(n+1)$-tupla de grupos normais. $O$ n-cubo de fibrações $B(\mathcal{G})$ é conexo se, e somente se, $n=1,2$ ou para todo $I, J \subseteq\langle n\rangle$ com $|I| \geq 2$ e $|J| \geq 1$ temos

$$
\left(\bigcap_{i \in I} N_{i}\right)\left(\prod_{j \in J} N_{j}\right)=\bigcap_{i \in I}\left(\prod_{j \in J \cup\{i\}} N_{j}\right)
$$

Demonstração: Sempre temos que

$$
\left(\bigcap_{i \in I} N_{i}\right)\left(\prod_{j \in J} N_{j}\right) \subseteq \bigcap_{i \in I}\left(\prod_{j \in J \cup\{i\}} N_{j}\right)
$$

e que temos a igualdade se $|I| \leq 1$ ou $|J|=0$, portanto sempre é verdade se $n=1$ ou $n=2$. Por 
indução no tamanho de $I$ temos que

$$
\operatorname{Im}\left(B(\mathcal{G})_{I, J} \rightarrow B(\mathcal{G})_{I, J \cup k}\right)=\left(\bigcap_{i \in I} N_{i}\right)\left(\prod_{j \in J \cup\{k\}} N_{j}\right)
$$

Logo o lema segue da sequência exata das fibrações $B(\mathcal{G})_{I \cup k, J} \rightarrow B(\mathcal{G})_{I, J} \rightarrow B(\mathcal{G})_{I, J \cup k}$.

$$
\text { Sendo } H_{n+1}\left(F ; R_{1}, \ldots, R_{n}\right)=H_{n+1}\left(B\left(\frac{F}{\prod_{i \in\langle n\rangle} R_{i}}\right) ; B\left(\frac{F}{\prod_{i \in\langle n\rangle \backslash\{k\}} R_{i}}\right): k \in\langle n\rangle\right) \text { temos o seguinte }
$$
corolário do teorema 4.3.2.

Corolario 4.3.4. Seja $F$ um grupo e $\left\{R_{i}\right\}_{i \in\langle n\rangle}$ uma familia de subgrupos normais de $F$ tais que $B\left(F ; R_{1}, \ldots, R_{n}\right)$ é conexo. Então existe um isomorfismo

$$
H_{n+1}\left(F ; R_{1}, \ldots, R_{n}\right) \cong \frac{\bigcap_{i \in\langle n\rangle} R_{i}}{\prod_{A \subseteq\langle n\rangle}\left[\bigcap_{i \in A} R_{i}, \bigcap_{i \notin A}^{\cap} R_{i}\right]}
$$

Com isso podemos agora derivar fórmulas para os grupos de homologia um grupo em dimensões arbitrárias análogas à fórmula de Hopf.

Teorema 4.3.5 (Fórmula de Hopf para $H_{n+1}(G)$ ). Sejam $F$ um grupo e $\left\{R_{i}\right\}_{i \in\langle n\rangle}$ uma família de subgrupos normais de $F$ tais que $B\left(F ; R_{1}, \ldots, R_{n}\right)$ é conexo e para cada subconjunto próprio $A \subset\langle n\rangle$ os grupos $H_{r}\left(\frac{F}{\prod_{i \in A} R_{i}}\right)$ são triviais para $r=2$ se $A=\emptyset$, e para $r=|A|+1$ e $|A|+2$ se $A \neq \emptyset$. Então se denotarmos $G=\frac{F}{\prod_{i \in\langle n\rangle} R_{i}}$ existe um isomorfismo

$$
H_{n+1}(G) \cong \frac{\bigcap_{i \in\langle n\rangle} R_{i} \cap[F, F]}{\prod_{A \subseteq\langle n\rangle}\left[\bigcap_{i \in A} R_{i},{ }_{i \notin A} R_{i}\right]}
$$

A hipótese que $B\left(F ; R_{1}, \ldots, R_{n}\right)$ deve ser conexo não foi incluída no artigo original [BE88], e contraexemplos para o enunciado original foram encontrados por outros autores. Um tal contraexemplo, assim como uma demonstração puramente algébrica desse resultado, pode ser encontrado no artigo de Donadze, Inassaridze e Porter [DIP05].

Demonstração: Essa fórmula segue do corolário e do fato que nas condições do teorema temos que a sequência

$$
0 \rightarrow H_{n+1}(G) \rightarrow H_{n+1}\left(F ; R_{1}, \ldots, R_{n}\right) \rightarrow H_{1}(F)=\frac{F}{[F, F]}
$$

é exata. Provaremos portanto a exatidão dessa sequência. Note que sempre temos por indução no tamanho de $B$ que a sequência

$$
\begin{aligned}
\cdots & \rightarrow H_{r}\left(\frac{F}{\prod_{j \in A} R_{j}} ; \frac{\prod_{j \in A \cup\{i\}} R_{j}}{\prod_{j \in A} R_{j}}: i \in B\right) \rightarrow H_{r}\left(\frac{F}{\prod_{j \in A \cup\{k\}} R_{j}} ; \frac{\prod_{j \in A \cup\{i, k\}} R_{j}}{\prod_{j \in A \cup\{k\}} R_{j}}: i \in B\right) \rightarrow \\
& \rightarrow H_{r}\left(\frac{F}{\prod_{j \in A} R_{j}} ; \frac{\prod_{j \in A \cup\{i\}} R_{j}}{\prod_{j \in A} R_{j}}: i \in B \cup\{k\}\right) \rightarrow H_{r-1}\left(\frac{F}{\prod_{j \in A} R_{j}} ; \frac{\prod_{j \in A \cup\{i\}} R_{j}}{\prod_{j \in A} R_{j}}: i \in B\right) \rightarrow \cdots
\end{aligned}
$$


é exata.

Isso implica que, como assumimos $H_{r}\left(\frac{F}{\prod_{i \in A} R_{i}}\right)$ triviais para $r=2$ se $A=\emptyset$, e para $r=|A|+$ 1 e $|A|+2$ se $A \neq \emptyset$, então temos por indução no tamanho de $B$ que se $A \cup B \neq\langle n\rangle$ então $H_{r}\left(\prod_{j \in A}^{F} R_{j} ; \frac{\prod_{j \in A \cup\{i\}} R_{j}}{\prod_{j \in A} R_{j}}: i \in B\right)$ é trivial se $r=|B|+2$ e $A=\emptyset$, e para $r=|A \cup B|+1$ e $|A \cup B|+2$ se $A \neq \emptyset$.

Temos portanto que se $A \cup B=\langle n\rangle$ e $A, B \neq \emptyset$ então

$$
H_{n+1}\left(\frac{F}{\prod_{j \in A} R_{j}} ; \frac{\prod_{j \in A \cup\{i\}} R_{j}}{\prod_{j \in A} R_{j}}: i \in B\right) \cong H_{n+1}\left(\frac{F}{\prod_{j \in A \backslash\{k\}} R_{j}} ; \frac{\prod_{j \in(A \backslash\{k\}) \cup\{i\}} R_{j}}{\prod_{j \in A \backslash\{k\}} R_{j}}: i \in B \cup\{k\}\right)
$$

e portanto por indução se $A \cup B=\langle n\rangle$ e $A \neq \emptyset$ então

$$
H_{n+1}(G) \cong H_{n+1}\left(\frac{F}{\prod_{j \in A} R_{j}} ; \frac{\prod_{j \in A \cup\{i\}} R_{j}}{\prod_{j \in A} R_{j}}: i \in B\right)
$$

Da sequência exata (4.1) também podemos concluir que se $B \neq\langle n\rangle$ e $k \in B$ então o homomorfismo

$$
H_{|B|+1}\left(F ; R_{i}: i \in B\right) \rightarrow H_{|B|}\left(F ; R_{i}: i \in B \backslash\{k\}\right)
$$

é uma injeção.

Finalmente podemos ver que temos a sequência exata

$$
0 \rightarrow H_{n+1}\left(\frac{F}{R_{n}} ; \frac{R_{1} R_{n}}{R_{n}}, \ldots, \frac{R_{n-1} R_{n}}{R_{n}}\right) \rightarrow H_{n+1}\left(F ; R_{1}, \ldots, R_{n}\right) \rightarrow H_{n}\left(F ; R_{1}, \ldots, R_{n-1}\right),
$$

pois vimos que $H_{n+1}\left(F ; R_{1}, \ldots, R_{n-1}\right)$ deve ser trivial. $\mathrm{O}$ que queríamos segue então de que $H_{n+1}(G) \cong H_{n+1}\left(\frac{F}{R_{n}} ; \frac{R_{1} R_{n}}{R_{n}}, \ldots, \frac{R_{n-1} R_{n}}{R_{n}}\right)$ por (4.2) e de que iterando (4.3) $n$ vezes obtemos que $H_{n}\left(F ; R_{1}, \ldots, R_{n-1}\right) \rightarrow H_{1}(F)$ é uma injeção.

Mostramos agora uma outra aplicação do teorema de excisão, agora para provar um resultado análogo ao teorema 4.1.3 sobre os grupos de homotopia relativo de espaços obtidos colando 2-células, provado originalmente por Whitehead. daremos uma descrição explicita do grupo de homotopia $\pi_{3}\left(X \underset{\lambda \in \Lambda}{\cup} e_{\lambda}^{3} ; U, V\right)$, aonde $X \underset{\lambda \in \Lambda}{\cup} e_{\lambda}^{3}$ é o espaço obtido de $X$ colando 3-células, a partir de informações contidas no 2-cubo cruzado de grupos $\Pi(X ; U, V)$ e das aplicações de colagem $f_{\lambda}$.

Teorema 4.3.6. Seja $X$ um espaço que é a união de abertos $U$ e $V$, aonde $U, V$ e $W=U \cap V$ são conexos e os pares $(U ; W)$ e $(V ; W)$ são 1-conexos. Sejam $f_{\lambda}:\left(\mathbb{S}^{2} ; E_{+}^{2}, E_{-}^{2}\right) \rightarrow(X ; U, V)$ uma família de aplicações pontuadas indexadas por $\lambda \in \Lambda$ e seja $X \underset{\lambda \in \Lambda}{\cup} e_{\lambda}^{3}$ o espaço obtido de $X$ colando 3-células via as aplicações $f_{\lambda}$. Temos então a seguinte descrição do grupo $\pi_{3}\left(X \cup_{\lambda \in \Lambda} e_{\lambda}^{3} ; U, V\right)$. Denote por

$$
\iota_{+} \in \pi_{2}\left(E_{+}^{2} ; \mathbb{S}^{1}\right), \quad \iota_{-} \in \pi_{2}\left(E_{-}^{2} ; \mathbb{S}^{1}\right), \quad \iota \in \pi_{1}\left(\mathbb{S}^{1}\right)
$$


os geradores dos seus respectivos grupos, aonde $\mu\left(\iota_{+}\right)=\mu\left(\iota_{-}\right)=\iota$, e por

$$
\begin{aligned}
& u_{\lambda}=f_{\lambda}\left(\iota_{+}\right) \in \pi_{2}(U, W) \\
& v_{\lambda}=f_{\lambda}\left(\iota_{-}\right) \in \pi_{2}(V, W) \\
& w_{\lambda}=f_{\lambda}(\iota) \in \pi_{1}(W)
\end{aligned}
$$

as imagens dos geradores. Seja $F(\phi) \rightarrow \pi_{1}(W)$ o módulo cruzado livre gerado pela função

$$
\begin{aligned}
\phi: \Lambda & \rightarrow \pi_{1}(W) \\
\lambda & \mapsto w_{\lambda}
\end{aligned}
$$

Então $\pi_{3}\left(X \cup_{\lambda \in \Lambda} e_{\lambda}^{3} ; U, V\right)$ é isomorfo ao quociente do produto livre de $F(\phi)$ e $\pi_{2}(U ; W) \otimes \pi_{2}(V ; W)$ pelas relações

$$
\begin{gathered}
{\left[(w, \lambda),\left(w, \lambda^{\prime}\right)\right]=\left({ }^{w} u_{\lambda} \otimes{ }^{w} v_{\lambda^{\prime}}\right)} \\
(u \otimes v)(w, \lambda)(u \otimes v)^{-1}=([\mu(u), \mu(v)] w, \lambda) \\
(w, \lambda)(u \otimes v)(w, \lambda)^{-1}=\left({ }^{w} w_{\lambda} w^{-1} u \otimes w_{\lambda} w^{-1} v\right)
\end{gathered}
$$

Demonstração: Seja $f: \underset{\lambda \in \Lambda}{\vee} \mathbb{S}^{2} \rightarrow X$ a aplicação determinada pelas aplicações $f_{\lambda}$, e seja $M_{f}$ seu cilindro de aplicação. Defina $Y=\left(M_{f} \cup \underset{\lambda \in \Lambda}{\vee} E_{\lambda}^{3}\right) / \sim$ aonde identificamos o bordo de $E_{\lambda}^{3} \operatorname{com}\{1\} \times \mathbb{S}_{\lambda}^{2}$. Então $Y$ possui um recobrimento $\left\{Z, B_{1}, B_{2}\right\}$ tal que $\left(Y ; B_{1}, B_{2}\right)$ é homotopicamente equivalente a $\left(X \underset{\lambda \in \Lambda}{\cup} e_{\lambda}^{3} ; U, V\right)$ e $\left(Z ; Z \cap B_{1}, Z \cap B_{2}\right)$ é homotopicamente equivalente a $\Sigma=\left(\underset{\lambda \in \Lambda}{\vee} E_{\lambda}^{3} ; \underset{\lambda \in \Lambda}{\vee}\left(E_{+}^{2}\right)_{\lambda}, \underset{\lambda \in \Lambda}{\vee}\left(E_{-}^{2}\right)_{\lambda}\right)$. Como o recobrimento $\left\{Z, B_{1}, B_{2}\right\}$ de $Y$ satisfaz as hipóteses do teorema de excisão 4.3.1 obtemos a apresentação de $\pi_{3}\left(X \underset{\lambda \in \Lambda}{\cup} e_{\lambda}^{3} ; U, V\right)$ dada pelo teorema 1.8.3 lembrando que para $F(\Lambda)$ o grupo livre com geradores os elementos de $\lambda$ temos que $\Pi(\Sigma)$ é o 2 -cubo cruzado

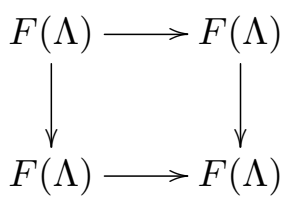

aonde todos os morfismos são a identidade e as funções $h$ são dadas por comutadores

\subsection{Colimites de espaços de classificação de grupos e os grupos de homotopia de $\mathbb{S}^{2}$}

Ellis e Mikhailov estudaram em [EM10] como calcular os grupos de homotopia de certos colimites de espaços de classificação de grupos. Usando ideias análogas a da seção anterior podemos construir a partir de uma $(n+1)$-tupla de grupos normais $\mathcal{G}=\left(G ; N_{1} \ldots, N_{n}\right)$ um $n$-cubo de fibrações $C(\mathcal{G})$ com $C(\mathcal{G})_{A, B}=B(\mathcal{G})_{A, B}$ quando $A \cup B \neq\langle n\rangle$, com

$$
C(\mathcal{G})_{\emptyset,\langle n\rangle}=\operatorname{colim}_{B \subset\langle n\rangle} B(\mathcal{G})_{\emptyset, B}
$$

e com os outros $C(\mathcal{G})_{A, B} \operatorname{com} A \cup B=\langle n\rangle$ dados indutivamente em $A$ como sendo a fibra de

$$
C(\mathcal{G})_{A \backslash\{k\}, B} \rightarrow C(\mathcal{G})_{A \backslash\{k\}, B \cup\{k\}} .
$$


Temos então nesse caso uma descrição alternativa dos grupos de homotopia do espaço $C(\mathcal{G})_{\emptyset,\langle n\rangle}$, em contraste da dada na seção $\mathbf{3 . 4}$.

Teorema 4.4.1. Sejam $\left\{N_{i}\right\}_{i \in\langle n\rangle}$ subgrupos normais de $G$ tais que os $(n-1)$-cubos de espaços $B\left(G ; N_{i}: i \in\langle n\rangle \backslash\{j\}\right)$ são conexos para cada $j \in\langle n\rangle$, e seja $X$ o espaço $C(\mathcal{G})_{\emptyset,\langle n\rangle}$. Então temos que

$$
\pi_{m}(X)=\frac{\pi_{1}(X)=\frac{G}{\prod_{i \in\langle n\rangle} N_{i}},}{\left(\bigcap_{a \in A} N_{a}\right)\left(\prod_{b \in B} N_{b}\right)},
$$

para $2 \leq m \leq n, A, B \subseteq\langle n\rangle,|A|=m$ e $B=\langle n\rangle \backslash A$, e que

$$
\pi_{n+1}(X) \cong \operatorname{Ker}\left(\pi_{1}\left(C(\mathcal{G})_{\langle n\rangle, \emptyset}\right) \rightarrow G\right)
$$

Demonstração: O grupo fundamental de $X$ segue do teorema de Seifert-van Kampen clássico.

Para $k \in\langle n\rangle, A \cup B=\langle n\rangle \backslash\{k\}$ e $m \geq 3$ temos, pela sequência exata da fibração e o fato que $C(\mathcal{G})_{A, B}$ é um espaço de classificação e portanto é um espaço de Eilenberg-MacLane do tipo $K(\pi, 1)$, que

$$
\begin{gathered}
\pi_{m}\left(C(\mathcal{G})_{A, B \cup\{k\}}\right) \cong \pi_{m-1}\left(C(\mathcal{G})_{A \cup\{k\}, B}\right) ; \\
\pi_{2}\left(C(\mathcal{G})_{A, B \cup\{k\}}\right) \cong \operatorname{Ker}\left(\pi_{1}\left(C(\mathcal{G})_{A \cup\{k\}, B}\right) \rightarrow \pi_{1}\left(C(\mathcal{G})_{A, B}\right)\right) ; \\
\pi_{1}\left(C(\mathcal{G})_{A, B \cup\{k\}}\right) \cong \frac{\pi_{1}\left(C(\mathcal{G})_{A, B}\right)}{\operatorname{Im}\left(\pi_{1}\left(C(\mathcal{G})_{A \cup\{k\}, B}\right) \rightarrow \pi_{1}\left(C(\mathcal{G})_{A, B}\right)\right)} .
\end{gathered}
$$

Pelo teorema 4.2.1, o lema 4.3.3 e (4.6) temos então

$$
\pi_{1}\left(C(\mathcal{G})_{A, B \cup\{k\}}\right) \cong \frac{\bigcap_{a \in A}\left(\prod_{b \in B \cup\{a\}} N_{b}\right)}{\prod_{\substack{I \cup J=A \cup\{k\} \\ I \cap J=\emptyset \\ I, J \neq \emptyset}}\left[\bigcap_{i \in I}\left(\prod_{b \in B \cup\{i\}} N_{b}\right), \bigcap_{j \in J}\left(\prod_{b \in B \cup\{j\}} N_{b}\right)\right]} .
$$

Como cada $\mu_{a}: \pi_{1}\left(C(\mathcal{G})_{A, B}\right) \rightarrow \pi_{1}\left(C(\mathcal{G})_{A \backslash\{a\}, B}\right)$ é uma inclusão temos por (4.5) que

$$
\begin{aligned}
& \pi_{2}\left(C(\mathcal{G})_{A, B \cup\{k\}}\right) \cong\left(\left(\underset{a \in A}{\circ} \mu_{a}\right)\left(C(\mathcal{G})_{A, B \cup\{k\}}\right)\right) \cap \operatorname{Ker}\left(\pi_{1}\left(C(\mathcal{G})_{\{k\}, B}\right) \rightarrow \pi_{1}\left(C(\mathcal{G})_{\emptyset, B}\right)\right)
\end{aligned}
$$

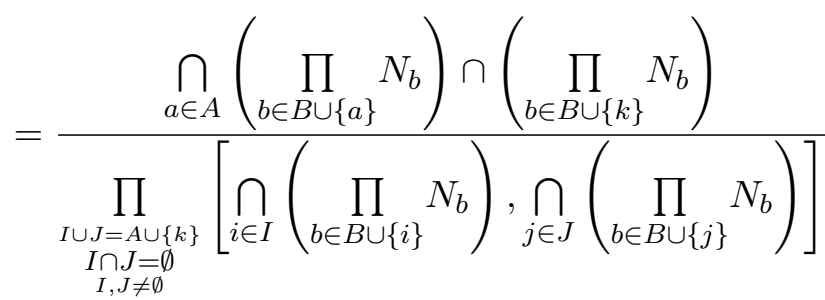




$$
=\frac{\bigcap_{a \in A \cup\{k\}}\left(\prod_{b \in B \cup\{a\}} N_{b}\right)}{\prod_{\substack{a \cup J=A \cup\{k\} \\ I \cap J=\emptyset \\ I, J \neq \emptyset}}\left[\bigcap_{i \in I}\left(\prod_{b \in B \cup\{i\}} N_{b}\right), \bigcap_{j \in J}\left(\prod_{b \in B \cup\{j\}} N_{b}\right)\right]}
$$

Assim pelo lema 4.3.3, usando a hipótese de conectividade, e por (4.4) e (4.8) obtemos a fórmula de $\pi_{m}(X)$ para $2 \leq m \leq n$ dada no teorema. A fórmula de $\pi_{n+1}(X)$ segue imediatamente de (4.4) e (4.5)

Vejamos agora como esse resultado nos permite encontrar uma fórmula para os grupos de homotopia da esfera de dimensão dois. Primeiro, precisaremos de uma definição. Lembre que para toda apresentação de grupos $\mathcal{P}=\langle X \mid \mathcal{R}\rangle$ podemos associar um CW-complexo de dimensão 2 dado por um bouquet de círculos indexados pelos elementos de $X$ e colando discos indexados pelos elementos de $\mathcal{R}$. Dizemos que uma apresentação de um grupo é aesférica se o CW-complexo associado a essa apresentação tiver os grupos de homotopia triviais em dimensões maiores que 1.

Definição 4.4.2. Uma apresentação $\mathcal{P}=\left\langle X \mid \mathcal{R}_{1}, \ldots, \mathcal{R}_{n}\right\rangle$ de um grupo é quase aesférica se ela não for aesférica e $\mathcal{P}=\left\langle X \mid \mathcal{R}_{i_{1}}, \ldots, \mathcal{R}_{i_{k}}\right\rangle$ é aesférica para todo subconjunto próprio $\left\{\mathcal{R}_{i_{1}}, \ldots, \mathcal{R}_{i_{k}}\right\} \subset$ $\left\{\mathcal{R}_{1}, \ldots, \mathcal{R}_{n}\right\}$

Considere uma apresentação quase aesférica do grupo trivial $\mathcal{P}=\left\langle X \mid r_{1}, \ldots, r_{n}\right\rangle$, e denote por $K_{\mathcal{P}}$ seu 2-complexo associado. O complexo de cadeia $C_{*} K_{\mathcal{P}}$ tem $C_{2} K_{\mathcal{P}}=\mathbb{Z}^{n+1}, C_{1} K_{\mathcal{P}}=\mathbb{Z}^{n}$ e um epimorfismo natural $C_{2} K_{\mathcal{P}} \rightarrow C_{1} K_{\mathcal{P}}$, cujo kernel é isomorfo a $H_{2}\left(K_{\mathcal{P}}\right)=\pi_{2}\left(K_{\mathcal{P}}\right)=\mathbb{Z}$, logo $K_{\mathcal{P}}$ é homotopicamente equivalente a $\mathbb{S}^{2}$.

Sendo $F$ o grupo livre nos geradores $x_{1}, \ldots, x_{n}$ e $R_{i}=\left\langle r_{i}\right\rangle^{F}$ temos a seguinte apresentação dos grupos de homotopia da esfera.

Teorema 4.4.3. Os grupo de homotopia da esfera $\mathbb{S}^{2}$ tem apresentação

$$
\pi_{n+1}\left(\mathbb{S}^{2}\right) \cong \frac{\bigcap_{k \in\langle n+1\rangle} R_{k}}{\prod_{\substack{I \cup J=\langle n+1\rangle \\ I \cap J=\emptyset \\ I, J \neq \emptyset}}\left[\bigcap_{i \in I} R_{i}, \bigcap_{j \in J} R_{j}\right]}
$$

Demonstração: Note que $\mathbb{S}^{2} \cong K_{\mathcal{P}}=C\left(F ; R_{i}: i \in\langle n+1\rangle\right)_{\langle n+1\rangle}$ e que por $\mathcal{P}$ ser quase aesférico esse cubo de fibrações é conexo. Logo a fórmula do teorema é consequência direta do teorema 4.4.1

Em particular tomando a apresentação quase aesférica $\mathcal{P}_{\mathrm{Wu}}=\left\langle x_{1}, \ldots, x_{n} \mid x_{1}, \ldots, x_{n}, x_{1} \ldots x_{n}\right\rangle$ recuperamos a fórmula encontrada por J. Wu[Wu01]. 



\section{Referências Bibliográficas}

[AS72] J.F. Adams e G.C. Shepherd, Algebraic Topology: A Student's Guide Cambridge University Press, London Mathematical Society Lecture Note Series, 4 (1972).

[BE88] R. Brown, G. Ellis, Hopf formulae for the higher homology of a group, Bulletin of the London Mathematical Society, 20.2 (1988), 124-128.

[BH78] R. Brown, P.J. Higgins, On the connection between the second relative homotopy groups of some related spaces, Proc. London Math. Soc., 36 (1978), 193-212.

[BHS11] R. Brown, P.J. Higgins, R. Sivera, Nonabelian Algebraic Topology: filtered spaces, crossed complexes, cubical homotopy groupoids, European Mathematical Society, 15 (2011).

[BL87a] R. Brown e J.L. Loday, van Kampen theorem for diagram of spaces, Topology, 26 (1987), 311-334.

[BL87b] R. Brown e J.L. Loday, Homotopical excision, and Hurewicz theorems for n-cubes of spaces, Proc. London Math. Soc. (3), 54 (1987), 176-192.

[BM51] A.L. Blakers e W.S. Massey, The homotopy groups of a triad (I), The Annals of Mathematics, 53.1 (1951), 161-205.

[BM52] A.L. Blakers e W.S. Massey, The homotopy groups of a triad (II), The Annals of Mathematics, 55.1 (1951), 192-201.

[BM53a] A.L. Blakers e W.S. Massey, Products in homotopy theory, The Annals of Mathematics, 58.2 (1953), 295-324.

[BM53b] A.L. Blakers e W.S. Massey, The homotopy groups of a triad (III), The Annals of Mathematics, 58.2 (1953) 409-417.

[Bro] Disponível em 〈http://pages.bangor.ac.uk/ mas010/nonabtens.html $\rangle \quad$, acesso em: $23 / 08 / 2013$.

[BW95] R. Brown, C.D. Wensley, On finite induced crossed modules and the homotopy 2-type of mapping cones, Theory Appl. Categ., 1 (1995), 51-74.

[DIP05] G. Donadze, N. Inassaridze, T. Porter, $n$-Fold Čech derived functors and generalised Hopf type formulas, K-theory, 35.3 (2005), 341-373.

[EH76] D.A. Edwards e H.M. Hasting, Čech and Steenrod homotopy Theories with Applications to Geometric Topology, Lecture Notes in Math., 542 (1976). 
[EM10] G. Ellis e R. Mikhailov, A colimit of classifying spaces, Advances in Math., 223 (2010), 2097-2113.

[ES87] G. Ellis e R. Steiner, Higher-dimensional crossed modules and the homotopy groups of ( $n+1)$ ads, J. Pure Applied Algebra, 46 (1987), 117-136.

[GJ99] P.G. Goerss e J.F. Jardine, Simplicial homotopy theory, Progress in mathematics, Springer, 174 (1999).

[GZ67] P. Gabriel, M. Zisman, Calculus of Fractions and Homotopy Theory, Ergebnisse der Mathematik und ihrer Grenzgebiete, Springer, 35 (1967).

[Hir09] P.S. Hirschhorn, Model categories and their localizations, AMS Bookstore, 99 (2009).

[Ka99] L.C. Kappe, Nonabelian tensor products of groups: the commutator connection, London Math. Soc. Lecture Note Series, (1999), 447-454.

[Lod82] J.L. Loday, Spaces with finitely many non-trivial homotopy groups, J. Pure Applied Algebra, 24 (1982), 179-202.

[Lur09] J. Lurie, Higher topos theory, Princeton University Press, 170 (2009).

[Mac98] S. Mac Lane, Categories for the working mathematician, Springer verlag, 5 (1998).

[May92] J.P. May, Simplicial objects in algebraic topology, University of Chicago Press, (1992).

[May99] J.P. May, A concise course in algebraic topology, University of Chicago Press, (1999).

[MW50] S. Mac Lane e J. Whitehead, On the 3-type of a complex, Proc. Nat. Acad. Sci., 36 (1950), 41-58.

[Po93] T. Porter, n-Types of simplicial groups and crossed n-cubes, Topology 32.1 (1993): 5-24.

[Qui67] D.G. Quillen, Homotopical algebra, Berlin: Springer-Verlag, 1967.

[St86] R. Steiner, Resolution of spaces by cubes of fibrations, J. London Math. Soc., (2) 34 (1986), 169-176.

[Wei95] C.A. Weibel, An introduction to homological algebra, Cambridge university press, 38 (1995).

[Whi41] J.H.C. Whitehead, On adding relations to homotopy groups, Annals of Math., 42 (1941), 409-428.

[Whi46] J.H.C. Whitehead, Note on a previous paper, Annals of Math., 47 (1946), 806-810.

[Whi49] J.H.C. Whitehead, Combinatorial homotopy II, Bull. Amer. Math. Soc, 55 (1949), 453-496.

[Wu01] J. Wu, Combinatorial description of homotopy groups of certain spaces, Math. Proc. Cambridge Phyl. Soc., 130 (2001), 489-513. 